\title{
WestVirginiaUniversity
}

THE RESEARCH REPOSITORY @ WVU

Graduate Theses, Dissertations, and Problem Reports

2017

\section{Corticospinal Excitability During Locomotion in Humans}

\author{
Lisa Kogan
}

Follow this and additional works at: https://researchrepository.wvu.edu/etd

\section{Recommended Citation}

Kogan, Lisa, "Corticospinal Excitability During Locomotion in Humans" (2017). Graduate Theses, Dissertations, and Problem Reports. 5997.

https://researchrepository.wvu.edu/etd/5997

This Thesis is protected by copyright and/or related rights. It has been brought to you by the The Research Repository @ WVU with permission from the rights-holder(s). You are free to use this Thesis in any way that is permitted by the copyright and related rights legislation that applies to your use. For other uses you must obtain permission from the rights-holder(s) directly, unless additional rights are indicated by a Creative Commons license in the record and/ or on the work itself. This Thesis has been accepted for inclusion in WVU Graduate Theses, Dissertations, and Problem Reports collection by an authorized administrator of The Research Repository @ WVU. For more information, please contact researchrepository@mail.wvu.edu. 


\title{
Corticospinal Excitability During Locomotion in Humans
}

\author{
Lisa Kogan
}

Thesis submitted

to the Benjamin M. Statler College of Engineering and Mineral Resources at West Virginia University

in partial fulfillment of the requirements for the degree of

Master of Science in Mechanical Engineering

\author{
Sergiy Yakovenko, Ph.D., Chair \\ Valeriya Gritsenko, Ph.D. \\ Yu Gu, Ph.D. \\ Department of Mechanical and Aerospace Engineering \\ Morgantown, West Virginia \\ 2017
}

Keywords: Transcranial magnetic stimulation, corticospinal excitability, motor evoked potentials, electromyography, locomotion.

Copyright 2017 Lisa Kogan 


\section{ABSTRACT \\ Corticospinal Excitability During Locomotion in Humans}

\section{Lisa Kogan}

The coordination between signals from cortical structures and spinal segmental pathways responsible for the control of locomotion remains a contentious issue in human motor control. The signals are known to be integrated, but the nature of these neural calculations is unknown. To understand these interactions in humans, noninvasive cortical stimulation techniques can be combined with detailed analyses of muscle activity patterns in locomotor tasks.

In this study, I tested the relationship between corticospinal inputs and the forward velocity of each limb. Transcranial magnetic stimulation (TMS) was used to elicit motor evoked potentials (MEPs) in 12 healthy human volunteers during locomotion on a split-belt treadmill, allowing for the evaluation of corticospinal excitability (CSE) throughout 4 gait tasks. Participants were instrumented with electromyography (EMG) sensors to collect activity of representative muscles of both lower limbs. The velocity conditions were limited to two symmetrical tasks, with both belts moving at either 1 or $1.25 \mathrm{~m} / \mathrm{s}$, and two asymmetrical tasks, with one belt moving at 1 and the other at $1.25 \mathrm{~m} / \mathrm{s}$. During each trial, a double cone coil was used to stimulate the area of the primary motor cortex (M1) associated with voluntary control of the lower limbs, throughout different phases of the step cycle. Real-time stimulation targeting was accomplished using tracking hardware and neuronavigation software, and the relative location of stimulation was automatically saved to ensure consistency between trials. The MEP epochs within the EMG signals were detected using an outgoing TMS synchronization pulse. Individual MEP magnitudes were normalized to the pre-stimulation muscle activity for comparison, and were binned according to step cycle phase.

Statistical tests were conducted using intra- and inter-subject group means from different velocity conditions, and phases of the step cycle. The relative locations of stimulation were also analyzed to describe the accuracy and precision of stimulation across trials. The non-normalized MEP amplitude was correlated with the muscle activity during control steps. The pattern of MEP modulation supported the hypothesis that CSE contains information about limb velocity. 


\section{Acknowledgements}

Firstly, I would like to thank my family and friends, both near and far, for their support throughout my time here in West Virginia.

This work could not have been conducted without the help of my lab mate Matthew Boots, who helped run all the experiments conducted for this work and was instrumental during the development of the experimental set up. Thank you also to the Neural Engineering Laboratory's technicians, Sarah Freeman and Neha Lal for their support. And of course, thank you to all my participants without whom this work would not have been possible.

Thank you to Bradley Pollard, Erienne Olesh, and Russell Hardesty of the Neural Engineering Research Laboratory for their help in setting up my experiment, discussing analysis strategies, and reviewing endless figures.

Thank you to Dr. Yu Gu and the entire WVU Sample Return Robot team. Your mentorship and friendship has been irreplaceable, and working with this team is an experience I will build on throughout my career. I feel lucky to have worked with you all.

Thank you to Dr. Valeriya Gritsenko for your direction in this and the other projects I explored in this lab, your willingness to answer questions, and of course for lending your TMS expertise and equipment for the experiment.

Finally, I would like to thank my adviser, Dr. Sergiy Yakovenko. Your guidance and support has made this thesis possible! Thank you for your enthusiasm and for welcoming me into your lab, and providing me with the freedom and encouragement to pursue projects and ideas, to share my work at conferences, and to learn at every opportunity. 


\section{Table of Contents}

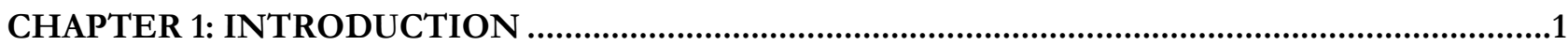

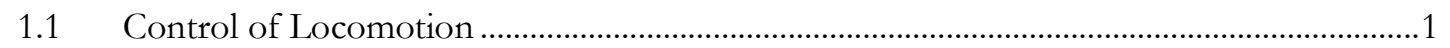

1.2 Corticospinal Excitability..................................................................................................

1.3 Thesis Outline and Contributions............................................................................................

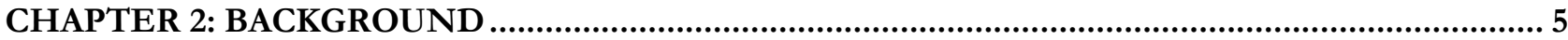

2.1 Locomotion in Humans ......................................................................................................

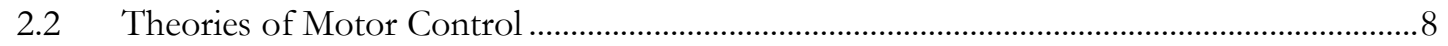

2.3 Corticospinal Excitability...................................................................................................

2.4 Signals Processing Techniques in Biomechanics ..................................................................16

CHAPTER 3: EVALUATING CORTICOSPINAL EXCITABILITY DURING LOCOMOTION ......20

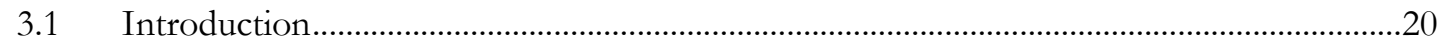

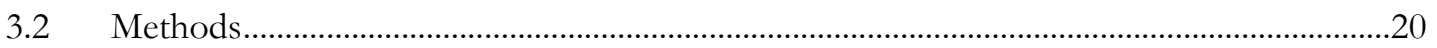

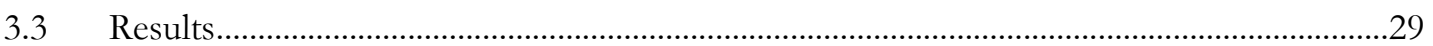

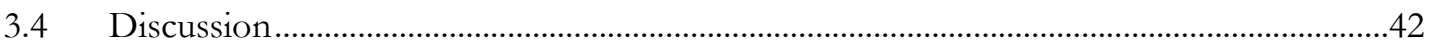

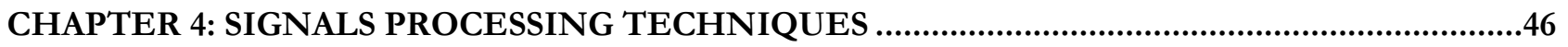

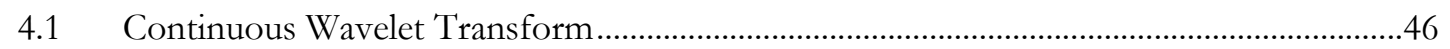

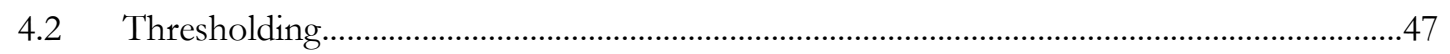

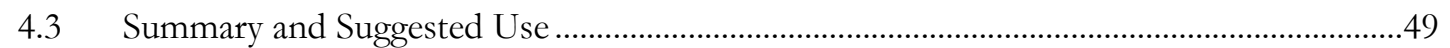

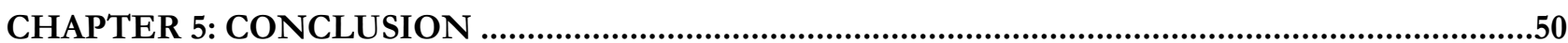

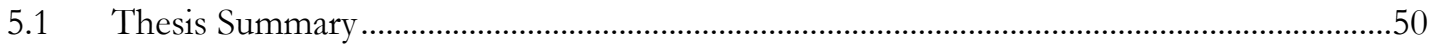

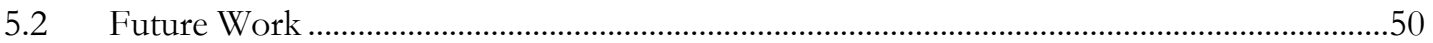

REFERENCES 


\section{List of Tables}

Table 1: Velocity conditions with left and right belt speeds defined.........................................................................20

Table 2: Velocity condition schedule for all subjects. Conditions are defined in Table 1.......................................21

Table 3: Legend for muscles instrumented with EMG sensors for each participant................................................22

Table 4: A summary of the average target and angular errors for each session. Data for subjects S02-S04 was not

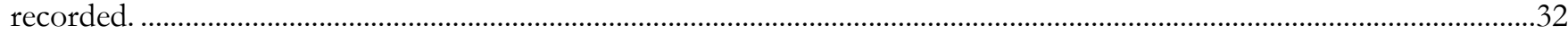

Table 5: ANOVA summary for normalized $\mathrm{MEP}_{\mathrm{pp}}$ magnitude...................................................................................34 


\section{List of Figures}

Figure 1: Simplified control diagram showing hypothesized velocity signal component $(V)$ affecting control signals from the corticospinal tract (CST) to central pattern generators (CPG). 2

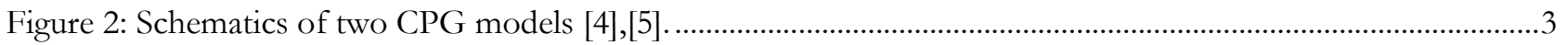

Figure 3: Left - a rendering of a Magstim $110 \mathrm{~mm}$ double cone coil. Right - the approximate region of stimulation,

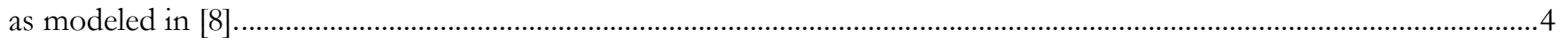

Figure 4: Schematic of locomotion in humans, with common gait terms presented, from Gage et al [50]..............6

Figure 5: Muscle activity data during treadmill walking from Buurke et al, 2016. [18] ...............................................7

Figure 6: Representative control diagram of Smith's predictor for an arbitrary system...........................................8

Figure 7: A schematic of a CPG structure producing extensor and flexor muscle group activity..............................

Figure 8: (a) The measured stance and swing times for a cat walking at increasing velocities. Middle: the CPG model used to determine the input required to produce the reciprocal muscle group activation leading to the measured cycles. (b) The velocity at which the data was required plotted against the predicted CPG input required [4]...............10

Figure 9: The results from Gilbreath et al, showing the success probably against the speed difference in interlimb velocity $[6] .$. . .11

Figure 10: A representation of cortical areas dedicated to particular function, also known as the homunculus [24].

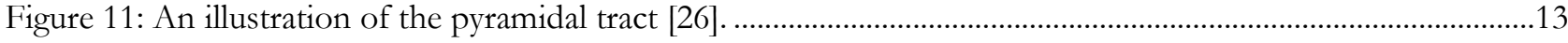

Figure 12: Schematic of experimental set-up (A) and protocol (B) [10]................................................................15

Figure 13: A. The experimental set-up used by Knikou et al [9]. E. The resulting MEP magnitude curves for the tibialis anterior.

Figure 14: Raw force plate data collected during locomotion, for ground reaction forces in the vertical direction.

Figure 15: Example of raw EMG data, collected during walking from the right tibialis anterior of a subject........17

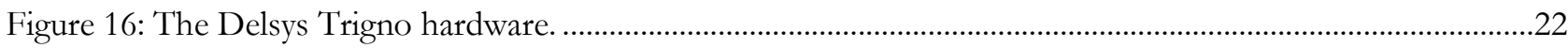

Figure 17: The Magstim double cone coil positioned over the vertex. .......................................................................23

Figure 18: A flowchart of the experimental triggering protocol................................................................................23

Figure 19: The hardware used for neuronavigation. On left, the subject array and coil array, affixed to the coil. On right, the Polaris camera and Rogue Research/Brainsight computer station....................................................................24

Figure 20: The locations used to locate the subject tracker to the MRI scan............................................................25

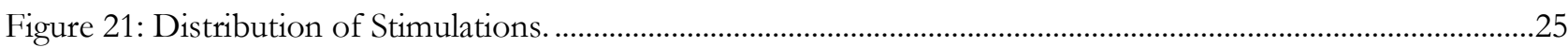

Figure 22: A flowchart describing part of the data processing pipeline, used to generate and save metadata identifying step onset and stimulation events, and separating control steps. ..............................................................26

Figure 23: MEP processing demonstrated for a single evoked response .................................................................27

Figure 24: Example ground reaction profiles for a single subject..............................................................................29 
Figure 25: Mean muscle activity during control steps, normalized to maximum EMG collected during control steps in each muscle. .30

Figure 26: Cross subject $(\mathrm{n}=12)$ primary muscle bursts for muscle activity in the left leg. .31

Figure 27: Screenshot showing a review of a Brainsight TMS session for a single participant. Stimulation locations are shown in orange in the 3D diagram, and indicated by the orange arrow in the sagittal view, demonstrating the spread of the stimulation locations. .32

Figure 28: Raw EMG data for the same MEP epoch in two different muscles. .33

Figure 29: An example of average MEP traces separated into each bin, or 20\% of the step cycle. .33

Figure 30: Average EMG traces for all MEPs for Subject 1, Trial Type 2, bin 4 of the step cycle. .34

Figure 31: MEP peak to peak amplitudes (black circles), normalized to maximum EMG amplitude within each signal. . .35

Figure 32: MEP peak to peak amplitudes (black circles), normalized to maximum EMG amplitude within each signal. . .36

Figure 33: MEP peak to peak amplitudes for all participants, normalized to muscle activity prior to stimulation.37

Figure 34: MEP peak to peak amplitudes for all participants, normalized to muscle activity prior to stimulation.38

Figure 35: Normalized MEP magnitudes in muscle groups of each leg, separated by left limb velocity. . .39

Figure 36: Normalized MEP magnitudes in muscle groups of each leg, separated by right limb velocity. .39

Figure 37: Plots of main effects of limb velocity on MEP magnitudes by muscle group for Bin 4: $21-40 \%$ of the step cycle. Inset plots show interaction between limb velocities.

Figure 38: Plots of main effects of limb velocity on MEP magnitudes by muscle group for Bin 4: 61-80\% of the step cycle. Inset plots show interaction between limb velocities . .41

Figure 39: Normalized MEP magnitude plotted at each phase of the step cycle. . .41

Figure 40: Example of implementation of automatic feature extraction method developed by Zhao.....................46

Figure 41: Example of MEP viewer, displaying detected latency and cortical silent period. .47

Figure 42: Histogram of MEP Latency values collected using the MEP viewer, for MEPs in the right tibialis anterior for subject 1 , for velocity condition 1 . 


\section{Glossary}

$\begin{array}{ll}\text { TMS } & \text { Transcranial Magnetic Stimulation } \\ \text { MEP } & \text { Motor Evoked Potential } \\ \text { CSE } & \text { Corticospinal Excitability } \\ \text { EMG } & \text { Electromyography } \\ \text { DAQ } & \text { Data Acquisition System } \\ \text { ANOVA } & \text { Analysis of Variance } \\ \text { CNS } & \text { Central Nervous System } \\ \text { CPG } & \text { Central Pattern Generator } \\ \text { M1 } & \text { Primary Motor Cortex } \\ \text { HAT } & \text { Head-Arms-Trunk }\end{array}$




\section{CHAPTER 1: INTRODUCTION}

The control of locomotion is a subfield of motor control with high relevance to human health, and has many open questions. Experimental and theoretical studies have provided detailed information on the transformations taking place in the distributed neuromusculoskeletal system of humans and lower animals; however, the control signals high in this hierarchy and contributing to coordination of muscle activity in the lower limbs across behavioral tasks are not fully defined. To understand how corticospinal inputs contribute to the behavior, the entire system between the primary motor cortex and the muscles of interest must be observed under the conditions that manipulate the parameters of interest.

The general state of the nervous system between the primary motor cortex (M1), and the motoneurons under its influence, is called the corticospinal excitability (CSE). Several methods exist to explore the behavior of this attribute, which can be thought of as a variable gain that modulates the response of a muscle to a cortical input. Transcranial magnetic stimulation (TMS) is a non-invasive method that can be used to provide a simultaneous input to a localized region of the M1 that will evoke a response in the muscles corresponding to that region. Using electromyography (EMG) recorded both with and without stimulation, and under different conditions, researchers can determine whether the gain is modulated according to the task being performed.

The objective of this work was to determine whether a velocity signal is represented within the descending input to the spinal control systems that generate locomotor patterns in humans. This task was completed by comparing the muscle responses evoked by stimulations applied to human participants during symmetrical and asymmetrical locomotor tasks imposed by a split-belt treadmill.

Two prior concepts, control of locomotion and corticospinal excitability, must be understood to complete the objective, and these are introduced here in brief. Further descriptions of these concepts and more general locomotion and signals processing techniques are presented in the Background section.

\subsection{Control of Locomotion}

The neural control of locomotion is a subfield of Systems Motor Control. Locomotion, which includes periodic tasks such as walking and running gaits, can be separated from fine motor control tasks wherein a person generates non-stereotypical movements such as during pointing with a finger, writing, or knitting. Locomotion is a stereotypical task that requires the coordination of multiple muscle groups both within each leg (intra-limb), and across both legs, trunk, and even arms (inter-limb). Such complex patterns are central for individual survival are known to rely on complex embedded and sensory feedback pathways [1]. The embedded components of this system are distributed between cortical, midbrain, and spinal neural circuits; however, the spinal rhythmogenic networks co-localized with motoneurons, which generate activation of muscles, have been shown to be sufficient for basic locomotor patterns.

These neural networks have been called Central Pattern Generators (CPGs) [2]. While cortical inputs are not essential for the generation of locomotor output, the predictive modulation of CPGs requires supraspinal inputs in behaviors such as the regulation of heading direction, or stepping over obstacles.

The control input to CPGs is clinically relevant, as locomotor function is often disrupted after neurological damage due to stroke and spinal cord injuries, among others. One of the general therapeutic 
approaches is locomotor rehabilitation based on the retraining of embedded circuits, or updating gains within the control system, to produce appropriate adaptations that compensate for missing or disrupted inputs or outputs. However, biomedical devices may soon provide improvements to these approaches. Advances in prototypes with cortical and spinal stimulating capabilities may lead to the development of implanted medical devices, similar to deep-brain stimulators, that re-animate or augment control of the lower limbs in people with locomotor impairments. These external inputs will be required to produce the stimulation patterns that are relevant to functional processing within the targeted biological system. That is, future cortical implants must be designed to address factors that affect the response to input according to different conditions.

In this thesis, I describe the experiment conducted to determine if there is a representation of limb velocity within descending pathways from the primary motor cortex. Specifically, the hypothesis was that the velocity of the lower limbs or the difference between limb velocities (inter-limb velocity), is related to corticospinal excitability during locomotion in humans.

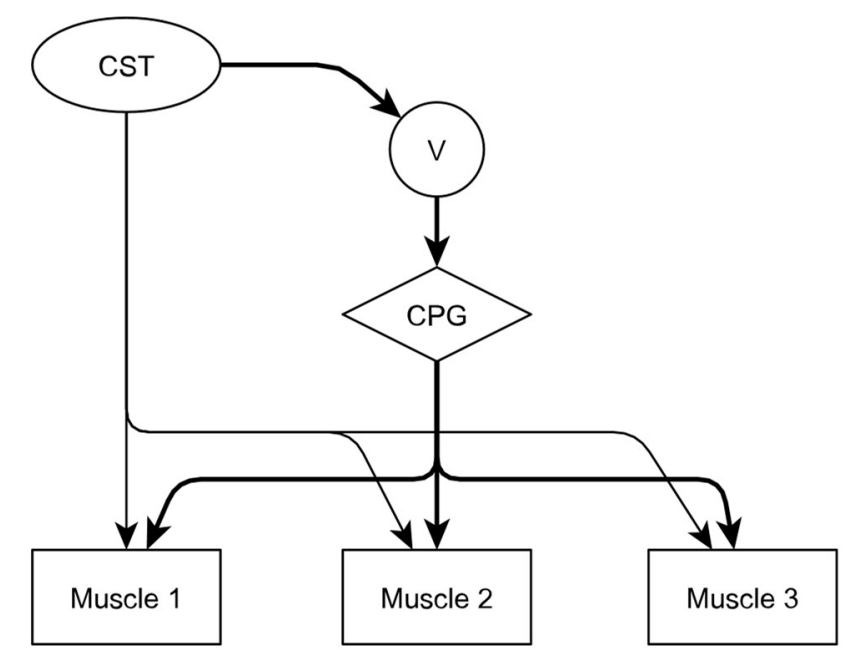

Figure 1: Simplified control diagram showing hypothesized velocity signal component (V) affecting control signals from the corticospinal tract (CST) to central pattern generators (CPG).

A schematic for a control diagram including the hypothesized velocity signal element is presented in Figure 1. The position of the velocity signal element within this control diagram is arbitrary and meant solely for illustration. Muscles receive inputs through direct pathways from the corticospinal tract (CST), and through localized biological control circuits in the spinal cord known as central pattern generators (CPGs), in addition to local reflex circuits. The signals integrated by CPGs may be modulated by limb velocity, or the overall speed of locomotion, by any group of neurons within these pathways.

\subsubsection{Central Pattern Generation}

Humans and other vertebrates such as cats and dogs have groups of cells that form biological control systems that coordinate periodic muscle activity such as locomotion. This simplifies the control of movement for the hierarchically high-level centers of the brain, as instead of commanding the various parameters regulating muscles individually, the gross control can be accomplished at a lower level. Central pattern generators (CPGs) are located in the spinal cord in humans [3]. CPGs have been modeled as leaky integrators that create the reciprocal activity between extensors and flexors leading to phasic contractions 
of the corresponding muscle groups and generating walking motion. Two examples are provided in Figure 2.
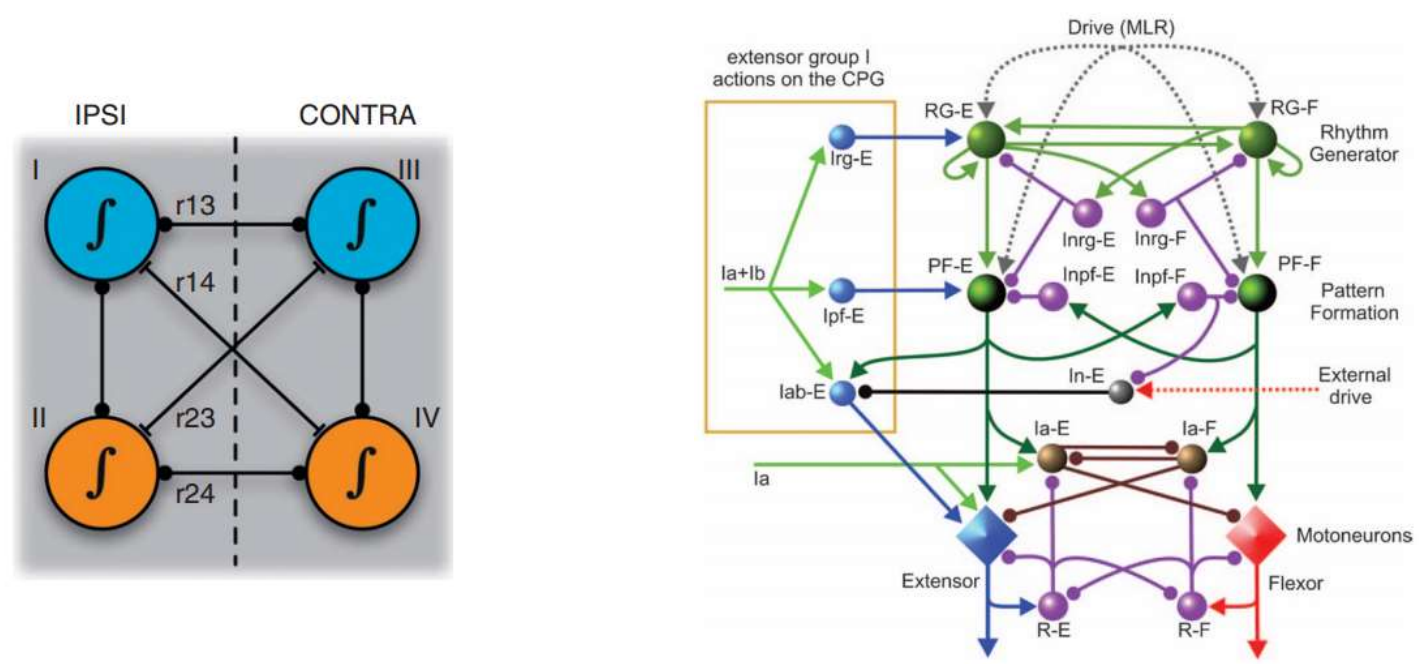

Figure 2: Schematics of two CPG models [4],[5].

While CPGs receive sensory feedback, also known as afferent signals, from biological sensors embedded into muscles, tendons, joints, and skin, these inputs are not essential for the detailed phasic output of these control elements, and existing models with varying complexity abound [4],[5].

\subsubsection{Representation of Velocity Signal}

Limb velocity may be one factor affecting the cortical input to CPGs. There are two main reasons for this hypothesis. The sensitivity of human perception to inter-limb velocity in walking has been explored using a split-belt treadmill [6]. Humans are able to discriminate between changes in inter-limb velocity (i.e. the difference between left and right leg velocity) to an accuracy of $1 \%$. The accurate detection of a physiological parameter under control is a required prerequisite to any physiological control system. Thus, the velocity of the lower limbs may be used as an input to corticospinal neural commands. In addition, the inverse solutions through the models of bilateral CPG (controlling two legs) validated by experimental data suggested that the inputs to each half-center are limb velocities. When the relative proportion of stance phase time to swing phase time was recorded for a cat walking at increasing velocities was placed into the CPG model presented in Figure 2 (on left), the resulting CPG input required to produce that motion was directly proportional to the velocity at which that data was collected [4].

\subsection{Corticospinal Excitability}

Corticospinal excitability (CSE) is the state of the central nervous system (CNS) between the primary motor cortex and the muscle being activated. The multitude of neurons making up this pathway is affected by the activity of every other connection or input to the network. Thus, if neurons that synapse onto a neuron in the pathway of interest are firing, and that connection is excitatory, that neuron is more likely to fire itself. This would represent an increased excitability. Conversely, if inhibitory connections are active, the manifestation of the decreased motor response to the same cortical input indicates a decrease in excitability. CSE is an overall measure of the level of neuronal excitability of the entire system. 
The method used to induce a cortical input for the main work of this thesis is transcranial magnetic stimulation.

\subsubsection{Transcranial Magnetic Stimulation}

Transcranial magnetic stimulation (TMS) employs a magnetic coil of specific geometry that, in conjunction with a large capacitor bank, is used to induce a transient current in a localized area of the brain. This approach is popular in human research and clinical practice because it is non-invasive and minimal risk in participants without contraindications such as a history of seizures or traumatic brain injury [7]. For TMS of the deep cortical structures associated with control of the lower limbs, a double cone coil, shown in Figure 3, should be used [8], according to its effective stimulation area.
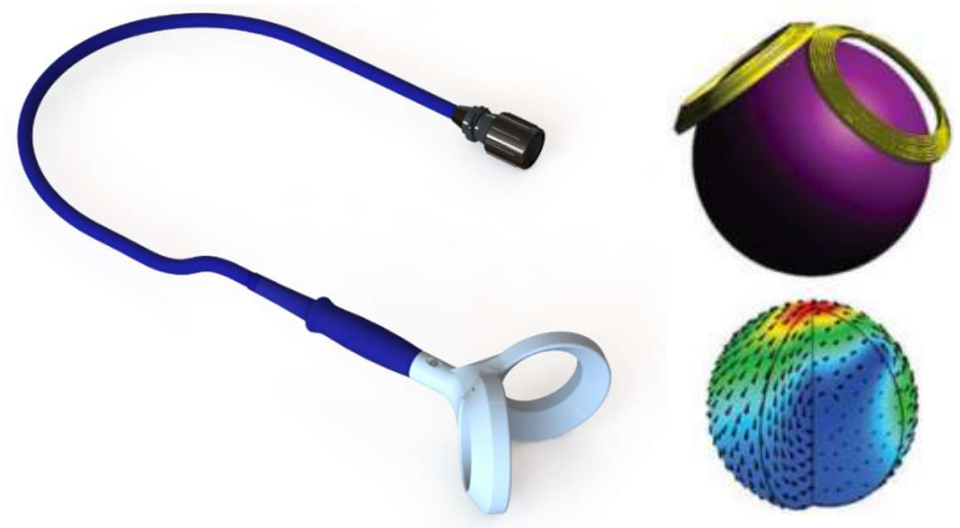

Figure 3: Left - a rendering of a Magstim $110 \mathrm{~mm}$ double cone coil. Right - the approximate region of stimulation, as modeled in [8].

Applying a stimulation in the same region consistently while a participant is walking is not a simple task, and harnesses have been used to increase precision of stimulation locations across trials [9], [10]. Improvements to the experimental protocol for TMS during locomotor tasks were explored in this study.

\subsection{Thesis Outline and Contributions}

The main contribution of this work is the evaluation of corticospinal excitability during human locomotion under varying limb velocity conditions. The hypothesis that limb velocity is represented within the descending input to the spinal control systems that generate locomotor patterns in humans was tested. A protocol for conducting transcranial magnetic stimulation studies in humans during walking on a treadmill was developed. Two methods used for processing electromyographic signals from such studies to best understand the underlying mechanisms under investigation were assessed.

Chapter 2 provides an overview of the necessary background information required to understand the methods, motivation, and results of the conducted experiment.

Chapter 3 describes the experimental design and development of the protocol for a locomotor TMS study, and presents results of this study in detail.

Chapter 4 is a brief discussion of processing techniques used to understand the electromyographic signals collected in the study.

Chapter 5 reviews the contributions of the thesis and presents a discussion of future direction for this research. 


\section{CHAPTER 2: BACKGROUND}

This chapter provides the background knowledge required to understand the major contribution of this thesis, the transcranial magnetic stimulation experiment described in Chapter 3.

To start, a primer on human locomotion is given. Basic parameters used to quantify and study walking patterns in humans are presented. Descriptions of how control models have helped improve understanding of human locomotion, and how models have been used to probe how the central nervous system (CNS) coordinates locomotor tasks, are also provided.

A general overview of motor control in humans is presented next. Corticospinal excitability is defined, and its importance in determining the relative contributions of task conditions is shown. The method used to evaluate corticospinal excitability within this thesis is described, and previous studies with similar experimental protocols are reviewed.

Finally, basic signals processing techniques used in biomechanical research are described, with focus placed on the sensor signals used within this work specifically. These signals include ground reaction forces, muscle activity via electromyography, and motion tracking signals for neuronavigation. Additional processing techniques for EMG data specific to quantifying motor evoked potentials are presented in this context as well.

\subsection{Locomotion in Humans}

Walking is a basic task that most humans are able to learn with the development of motor coordination during early childhood. Moving from location to location is a fundamental aspect of dayto-day living for most people, and mobility is a measure of quality of life [11].

\subsubsection{Basic Parameters}

While the study of locomotor dynamics can be dated back to ancient times, it is important to note the identification of neural elements involved in locomotor control by Sir Charles Sherrington [12] and his student Thomas G. Brown [2]. In a separate effort, motion analysis has been pioneered by Edward Muybridge, who was the first to collect the high-speed kinematic evidence of different locomotor phases in bipeds and quadrupeds. In 1905, Phillipson used this analysis to create an exhaustive description of locomotor phases and their modulation with the speed of locomotion[13].

In recent decades, the quantitative study of walking was greatly advanced by David A. Winter. Winter worked to define human locomotion and basic parameters to enable future study [14]. By recruiting participants with normal, pathological, and aging gait, Winter established the first datasets of basic parameters of all three types of walking, and created a benchmark with which to evaluate gait in humans. In this first work, basic instrumentation such as goniometers were used to measure kinematic variables, specifically joint angles.

These studies identify several key basic parameters of gait that systematically characterize spatiotemporal aspects of gait associated with stride, step cycle, stance, and swing. 


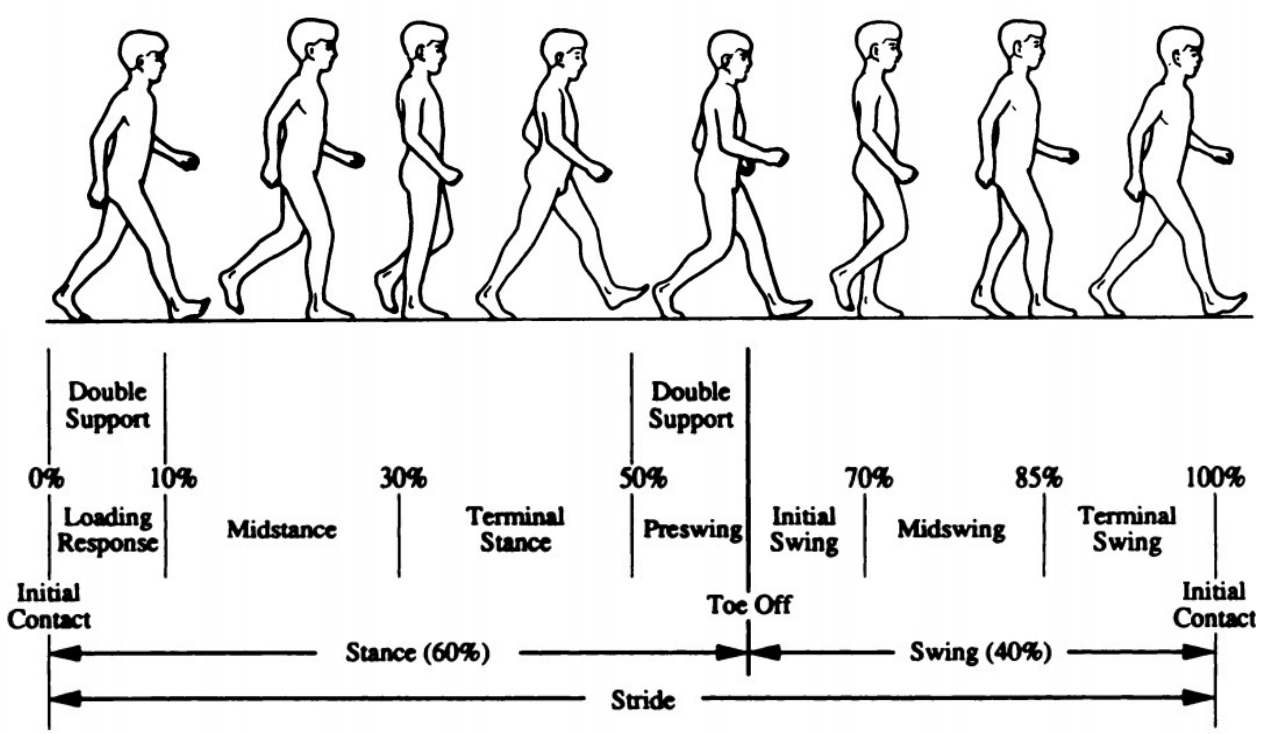

Figure 4: Schematic of locomotion in humans, with common gait terms presented, from Gage et al [50].

Symmetrical gait can be separated into the action of each individual limb, as shown in Figure 4. For regular human walking gait, stance comprises about $60 \%$ of the step cycle, and swing comprises $40 \%$ of the step cycle, for each limb. Because of this emphasis on stance, there is an overlap of the stance phases of the two limbs, that is, human locomotion has a double stance or support phase. This is used to define walking over other locomotor tasks such as running or hopping, where there is no double stance phase.

2.1.2

Kinematics

The kinematics of walking largely focus on the angles of the joints involved: the hip angles (flexion, extension, abduction/adduction, and pronation/supination), knee angles (flexion/extension), and ankle angles (plantarflexion and dorsiflexion). Most the range of motion of these joints can be described with the measures described in a $2 \mathrm{D}$ plane, however variations exist between participants for out-of-plane movements.

\subsubsection{Dynamics}

Descriptions of the dynamics of walking include joint moments and torques, and other reactive forces such as ground reaction forces (GRFs). GRFs are easily measurable and can be used to extract relevant information about balance, position of the center of mass, and step onset and offset. They are comprised of a set of six parameters including three axis force, and three-axis moments that can be measured with the use of force-plates instrumented into treadmills.

Dynamic walking can be achieved with a so-called Passive-Dynamic Walker. This means that walking is achieved without a controller, simply due to the properties of the elements comprising the walking system [15].

Note that variations exist in the way different people walk. This can be due to several factors, including different constraints during the development of gait, individual biomechanical constraints, and the expertise of walking. One of the predominant explanations for gait variance is rooted in the concept of uncontrolled manifold theory [16] and optimal feedback control [17]. The variance may arise from the lack of stabilization for parameters that are not crucial for the behavior. Instead of total control of all 
possible variables, which may be costly, only meaningful parameters are actively monitored. In a dynamic system, these unconstrained parameters may cause behaviorally insignificant deviations from the stereotypical pattern.

Thus, variations are expected even for steps taken at the same speed, and may carry additional information about the organization of locomotor control. In this study, we used multiple observations to standardize the behavior to account for these variations. In order to quantify these differences, data is typically normalized to the step cycle starting from heel onset to directly prior to the subsequent heel onset.

Winter described three tasks to one of the challenges of walking for humans as the distribution of mass vertically. The head, arms, and trunk (HAT) comprise two thirds of body weight and are two thirds above the ground, in an inverted pendulum configuration. The torques produced around the hip must therefore compensate for the position of the HAT to stabilize and keep the body upright. Another problem the CNS must solve during walking is keeping the body from collapsing.

The double inverted pendulum model has been used to calculate the resultant moments about the joints of the lower limbs involved in walking (the hip, knee, and ankle) by solving for the derived dynamic equilibrium equations. These equations can be used in conjunction with measured force plate data to estimate actual moments about these joints in an experimental setting [14]

\subsubsection{Muscle Activity}

Muscle activity during normal, symmetrical walking has also been recorded using EMG sensors to determine the temporal patterns of muscle activity at different times of the step cycle. The following traces show average muscle activity normalized to the length of individual step cycles for eight muscles representing the main flexor, extensor, and bifunctional muscle groups during treadmill walking at different walking speeds.
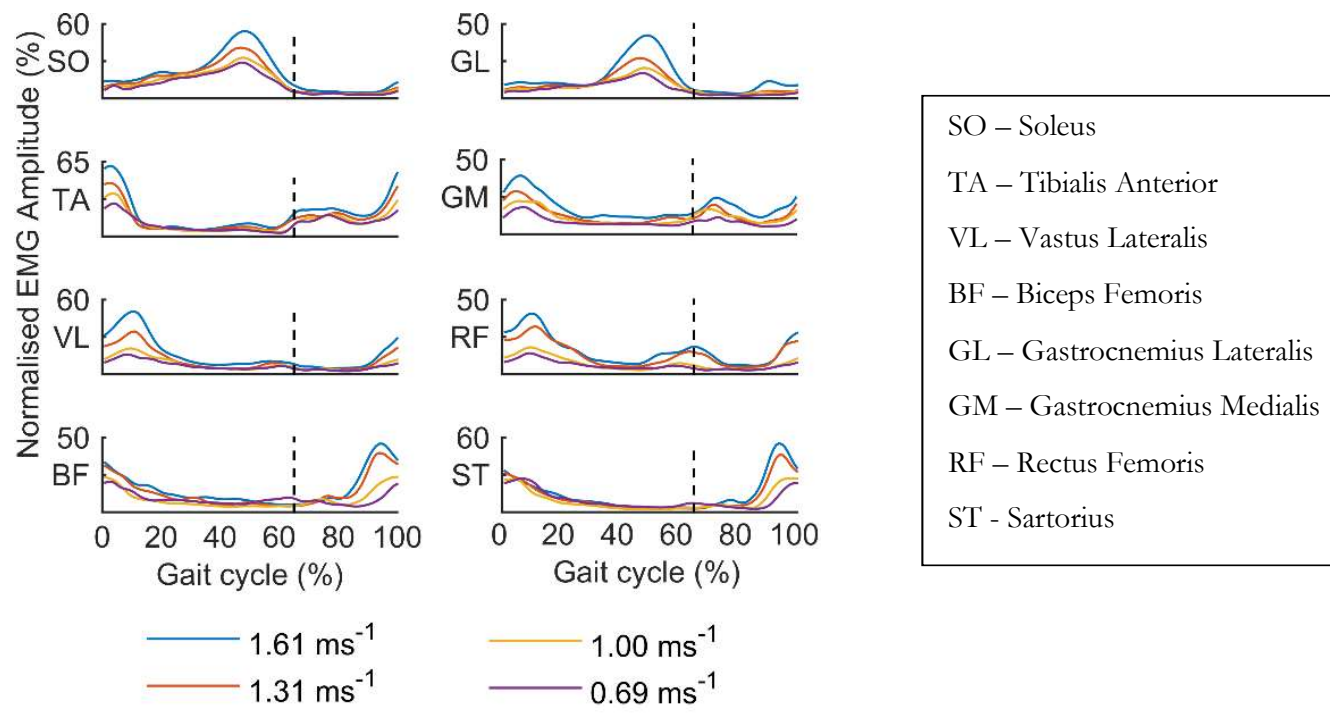

Figure 5: Muscle activity data during treadmill walking from Buurke et al, 2016. [18] 
Motor control is the control required to produce any voluntary action of the musculoskeletal system. Understanding how the human brain coordinates muscle activity to produce desired movement is as complex as the task itself. Much has been elucidated by the field of neuroscience and neural and musculoskeletal physiology in order to identify the cascading systems involved in producing motion reliably and accurately. This includes the description of both efferent (feedforward) and afferent (sensory feedback) pathways, as well as physiological properties of biological tissue which impacts musculoskeletal dynamics. However, the manner in which the brain integrates this information to create top-down control is still being investigated. The pursuit of the mechanistic level of understanding drives the creation of high-fidelity, biomimetic musculoskeletal models and the control schema required to control them in a suitable manner.

In this section, several control theories used in describing the biomechanics and control of human locomotion are introduced, and the rationale for exploring the relationship between limb velocity and corticospinal excitability is provided.

\section{Smith's Predictor \& Internal Models}

In 1957, Otto Smith introduced a method of control termed posicast, for positive cast, which has since been more commonly referred to as Smith's Predictor [19]. Smith's predictor is a method for controlling lightly-damped oscillatory systems in such a way as to minimize the number of time steps to reach the desired output signal, with a minimum rise and settling time, and less than $2 \%$ overshoot/undershoot. That is, it produces a dead-beat response in the ideal case. This is accomplished by adding a transient component to the plant input that is created to compensate for the initial input to the plant, such that the schedule of inputs to the plant will produce optimal movement.

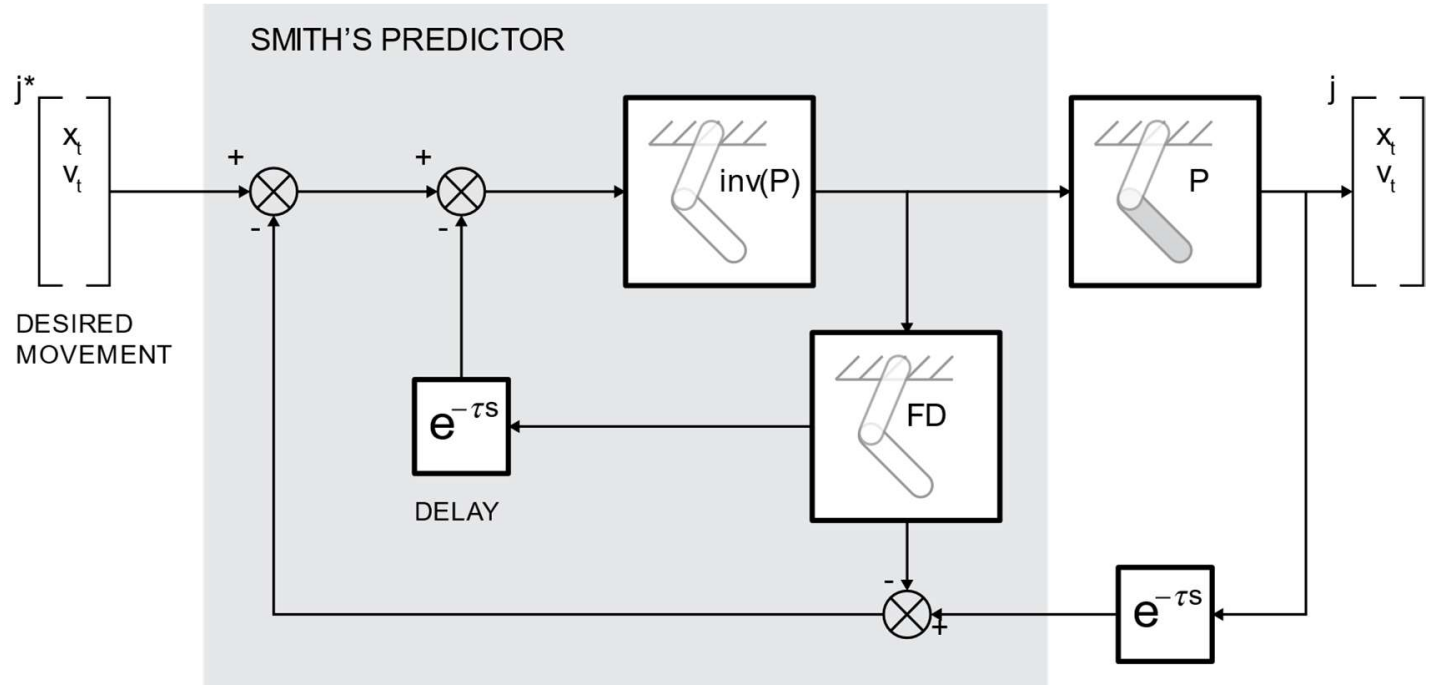

Figure 6: Representative control diagram of Smith's predictor for an arbitrary system.

In order to implement Smith's predictor to a particular plant, one must probe the plant to create a model. The more accurate the model plant is to the real plant, the more optimal, that is, the more efficient the final control of the plant will be. 
Smith's predictor provides a helpful means of controlling complex feedback systems in general, and in biomechanics, can be used to better understand motor control in mammals. The structure of Smith's predictor has been used to posit the existence of an internal model through which the primary motor cortex is able to moderate and appropriately schedule inputs to the musculoskeletal system. In the human analogue, the brain can be thought of as having an internal model which is used to apply an appropriate sequence of motor inputs, as opposed to relying purely on feedforward or feedback control.

Similar control filters relying on internal models have been shown to function successfully, as evidenced by the proliferation of Kalman filters [20].

\subsubsection{Central Pattern Generation}

Locomotion specifically provides additional complications. The act of taking one step may be coordinated directly prior to taking that step; however, for steady gait and other rhythmic motions, there are neural circuits which will produce the periodic commands necessary to produce the desired locomotor pattern. These dedicated spinal circuits are called Central Pattern Generators, or CPGs, and can function purely as feedforward components of a larger model. There are local sensory circuits, called segmental reflexes, which can also produce rhythmic motion provided the muscles are already being perturbed. The complexity of these feedback mechanisms and their pervasive action during movement have obscured the existence of CPGs even after their discovery by Thomas G. Brown in 1911 [21].

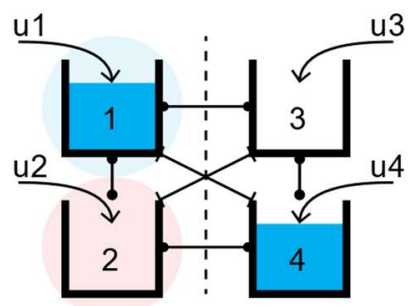

Figure 7: A schematic of a CPG structure producing extensor and flexor muscle group activity.

Arthur D. Kuo explored the contribution of both these physiological feedforward and feedback circuits to the control of rhythmic motion through the use of a damped pendulum model [20]. Since both circuits can be tuned to act identically, the relative contribution of each depends on the effect of unexpected perturbations to the system (greater effect on feedforward behavior), and the accuracy of the sensors (greater effect on feedback behavior). Kuo created a hybrid feedback/feedforward model to investigate whether the combination provided an advantage to either system independently. The results for the simple pendulum model demonstrate that for systems subject to both measurement noise and perturbations, such as human locomotion, there is an optimal combination of feedback and feedforward components that will result in better performance than either component alone.

Another aspect of motor control that must be addressed is the redundancy of the system, or Bernstein's problem [22]. The same end-point kinematics can be produced by multiple joint excursion combinations; moreover, joint movement can be achieved with different actions of muscles that span these joints. Yet humans produce the same task in without using the same temporal activation profile.

Emmanuel Todorov described a method to reconcile this redundancy and accurate task completion by suggesting a theory of motor coordination known as Optimal Feedback Control. In this theory, the 
optimal strategy for controlling such an uncertain and redundant system is to allow for variability in the task-independent dimensions of the system. The solution is then constrained by the task performance that creates system behavior that is only corrected for if it causes significant deviation from the task objective (goal-oriented corrections) [17].

\subsubsection{Velocity Signal}

A model of a CPG was developed in our laboratory to determine the nature of signals that drive this biological element using inverse solutions [4]. Stance and swing times measured from data recorded during locomotion by a cat was collected at increasing gait velocities. The relative proportion of stance and swing times was used as an input to the CPG model shown in Figure 8 in order to predict what level of CPG input (in arbitrary units) would produce the reciprocal muscle group activity necessary to produce the gait in the collected data. The original velocity at which the cat was walking was highly linearly correlated $\left(\mathrm{R}^{2}=0.9978\right)$ with the computed CPG input. This result suggest velocity may be represented within the input to CPGs.
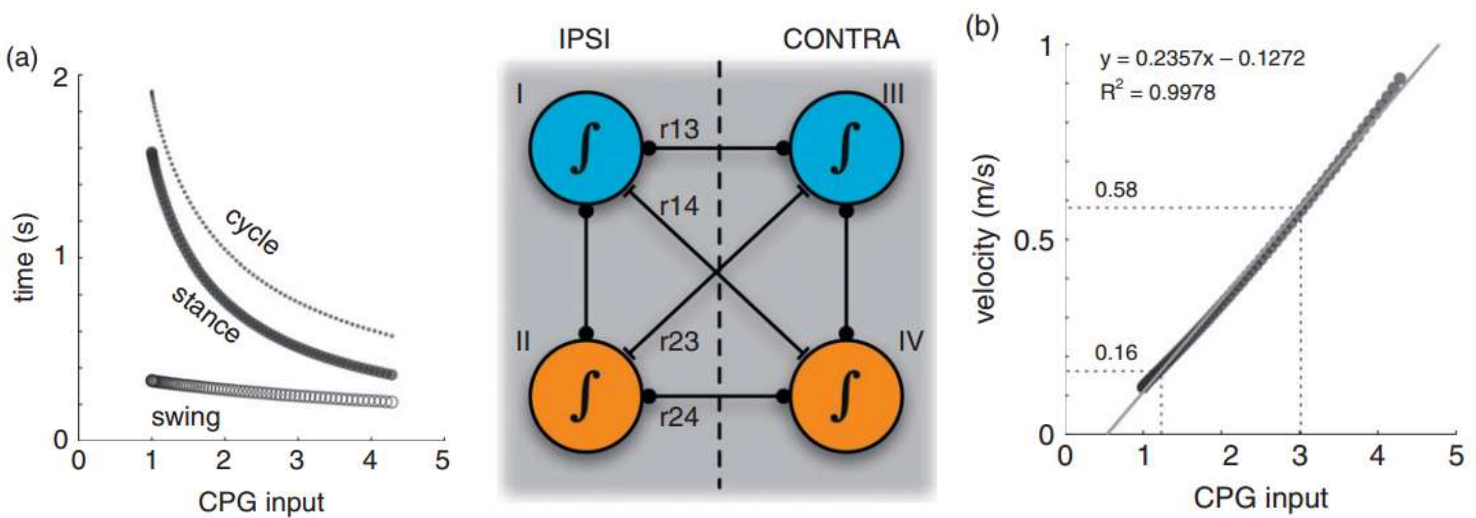

Figure 8: (a) The measured stance and swing times for a cat walking at increasing velocities. Middle: the CPG model used to determine the input required to produce the reciprocal muscle group activation leading to the measured cycles. (b) The velocity at which the data was required plotted against the predicted CPG input required [4].

Finally, the results of Gilbreath et al in 2015 demonstrated that humans are able to discern interlimb velocity with a difference of 1\% [6]. During an interlimb velocity discrimination task using a splitbelt treadmill, participants were asked to walk for $3 \mathrm{~s}$ prior to each trial, after which one of either the left or right belt speeds was changed to $1 \mathrm{~m} / \mathrm{s}+/-0.01,0.02,0.05,0.1$, or $0.2 \mathrm{~m} / \mathrm{s}$, or kept the same throughout. The participants were then asked to verbally report which belt was moving faster at the onset of a tone indicating the belt speeds had changed, whether or not they had.

This experiment was repeated under 110\% loaded and 90\% unloaded conditions using a backpack with weights, and an unweighting harness system, respectively. Under these conditions, participants were still able to identify the correct limb with a $1 \%$ difference in velocity, as shown in Figure 9. 


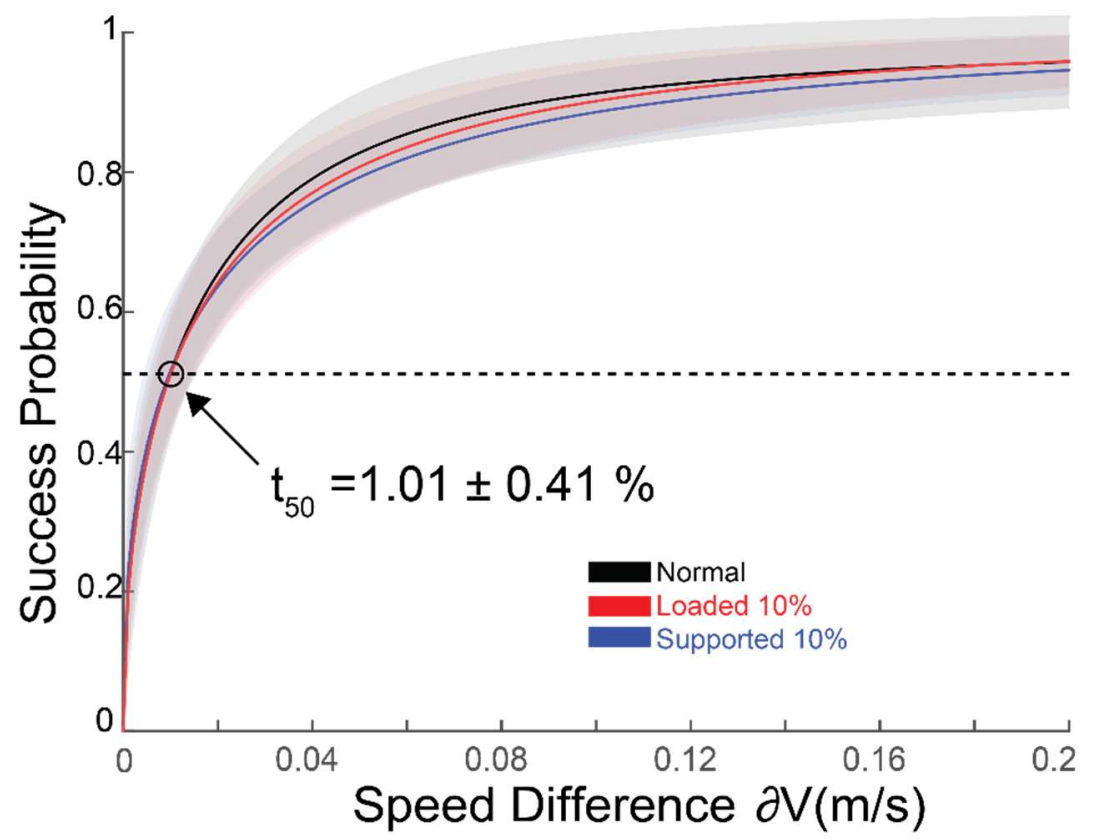

Figure 9: The results from Gilbreath et al, showing the success probably against the speed difference in interlimb velocity [6].

The conscious awareness of this variable suggests that the velocity of limbs, as captured by the speed of locomotion, may provide input to the control signal for human locomotion. Additionally, since the proprioceptive information (loading, body weight support, or lack thereof) did not influence the ability to detect interlimb velocity differences, this result also suggests velocity information may be extracted at the spinal level. The significance of such an input would mean that there is a higher order sense of velocity that is encoded in the brain for control of locomotion.

\subsubsection{Theories of Motor Control Summary}

Theories of motor control suggest that there is an internal model for each task humans complete. For locomotion, such an internal model will include the contribution of biological control circuits such as CPGs, but the various inputs to this internal model are not known. An inverse solution for input using recorded data and a CPG model has suggested that velocity may influence the input to CPGs in cats. The ability of humans to detect small differences in inter-limb velocity suggests that the higher-level measures of the velocity of locomotion may be encoded for in the control of locomotion.

\subsection{Corticospinal Excitability}

Corticospinal excitability (CSE) refers to the gross measurement of gain between the motor cortex and the muscles being contracted. As described above, there are many systems the signal must pass through before reaching the muscle. The gain can be further split up into two sequential transformations: cortical gain, and spinal gain.

For an identical cortical input, corticospinal excitability dictates the resulting level of activation of a muscle. This input-output relationship between cortical input and resulting motor activity has been explored by using such a set-up, and applying a simultaneous stimulation to the M1, and measuring the muscle activity that is evoked due to that stimulation. The measured signal is termed a motor evoked potential, or MEP, and can be evoked using corticomedullary stimulation, electrical stimulation, and most relevantly, through the use of transcranial magnetic stimulation. 
CSE has been shown to be task dependent, and can even change due in stationary participants watching videos of actors carrying out tasks which involve the muscles being investigated [23]. This means care must be taken to control the visual input provided to participants during TMS studies, so as not to bias the data by showing videos of people completing motor tasks.

\subsubsection{Neurophysiology}

Neurophysiology encompasses the physiology, or functional aspects, of the entire nervous system. The basic unit of the nervous system is the neuron. Neurons are specialized cells in the body that transmit signals to other cells. Neurons are connected to each other and other types of cells by synapses to form a network. A neuron integrates the signals received from these various connections, and if a membrane potential is reached, ion gates will open and cause a depolarization of that neuron, which causes the neuron to "fire". In turn, the neurons onto which that neuron synapses will receive an input in the form of a change in electrical potential, and the cycle repeats [24].

\subsubsection{Central Nervous System (CNS)}

The CNS is the entire collection of nervous tissue that controls the activity of the body in animals. In humans, this complex includes the brain, spinal cord, and all other nervous tissue where neurons are found. Their function can be simplified (for explanatory purposes) as being digital, either they transmit a signal, or "fire", or they do not, and they do so at a certain frequency dependent on the surrounding conditions and inputs [24]. Since neurons will produce an output based on a combination of inputs, they can be described as biological signal integrators. Different types of neurons may synapse onto one or more other neurons, or different kinds of cells entirely. Additionally, some connections may be inhibitory, that is they will decrease the likelihood of a neuron firing, or excitatory, increasing the likelihood of a neuron firing.

Neurons are interconnected, and the networks that these simple units form are responsible for the complex behaviors and activities that humans exhibit. In the context of motor control, a series of connections that results in a certain behavior is called a pathway.

\subsubsection{Motor Cortex \& Muscle Activity}

The primary motor cortex is the region of the brain responsible for voluntary movement in animals. A motor homunculus, or representation of the regions of the body controlled by the regions of the brain, is provided in Figure 10 below.

Muscles are tissues, or collections of cells, which contract when electrically stimulated. Muscle activity is the result of the integration of descending commands from the motor cortex, and the activity of local circuits, such as reflexes. In some cases, a descending command may override these reflexes, for example as in the case of another person hitting your arm while you are holding a cup of coffee or an unstable load [25]. The final neuron that provides stimulation to a group of muscle cells is called a motor neuron, and the collection of a motor neuron and the muscle cells it activates is called a motor unit [24].

Muscle activity can be measured non-invasively using surface electromyography (EMG) sensors. EMG sensors measure the voltage potential difference between two to four pads, and are effectively recording the sum of motor unit action potentials. The polarity of the recorded data is arbitrary, and depends on the orientation of the sensor electrodes relative to the longitudinal direction of the muscle. 


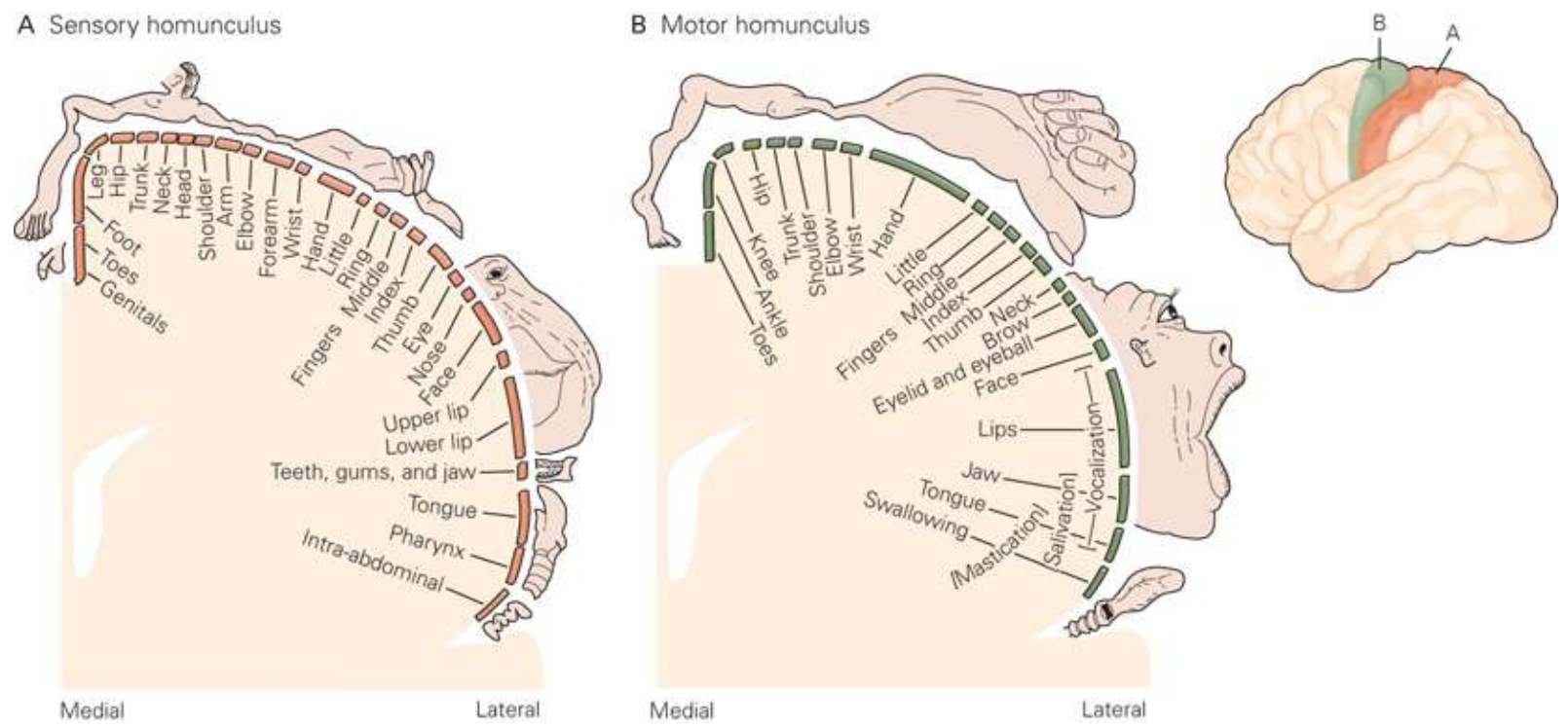

Figure 10: A representation of cortical areas dedicated to particular function, also known as the homunculus [24].

An input to, and therefore from, the region associated with the lower limbs will evoke a motor response in the muscles of the lower limbs. Figure 11 is an illustration of the pyramidal tract, and demonstrates the complexity and structure of the pathway through cortical signals must pass to activate a muscle or group of motor units. The state of the network comprising this pathway is the CSE.

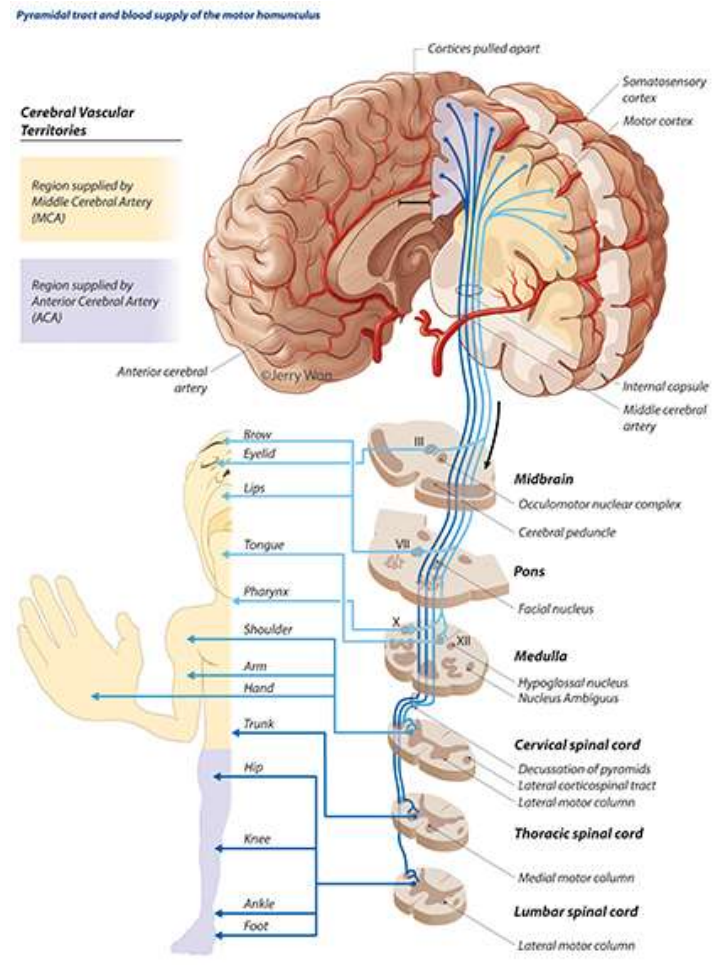

Figure 11: An illustration of the pyramidal tract [26]. 
Transcranial magnetic stimulation (TMS) is a method of applying a simultaneous input to a small area in the brain in a non-invasive and safe fashion. TMS works on the principle of electromagnetic induction. A basic TMS system includes control apparatus to select stimulation intensity and trigger stimulation, a capacitor bank to store charge, and a magnetic coil of specific geometry. When a stimulation is triggered, the capacitor bank is discharged into the magnetic coil, and an electrical current is induced in the area relative to the coil surface determined by the geometry of the coil. The direction of current produced depends on the coil configuration, and to the shape of the current discharged through the coil.

Using a specific coil that induces a current further away from the coil surface, experimenters can stimulate areas of the brain further from the skull. This is useful for stimulation the M1 area responsible for controlling the lower limbs. The coil used for conducting TMS experiments in the lower limbs is a double cone coil, $110 \mathrm{~mm}[8]$.

In other applications, repetitive TMS has been used to encourage a temporary or virtual lesion of a region of the brain in order to study brain-behavioral relationships [27]. Repetitive TMS has also been explored for use in treatment of depression [28].

\subsubsection{2}

\section{Locomotor Studies}

TMS has been used to study corticospinal excitability in humans during locomotion with different tasks as early as 1999 [29]. Experiments from that time relied on a set metronome beat to normalize participant gait, with varying treadmill velocity depending on the preferred stride length for each participant. Schubert et al conducted an experiment to determine the behavior of TMS induced MEPs during a precision stepping task, and their results supported the hypothesis that volition has a conditioning effect on the MEPs during stepping [29]. The background EMG activity was used to normalize MEP magnitudes, which have been shown known to correlate to muscle activity [10]. Significant differences between means of these normalized magnitudes were attributed to the conditions under which the responses were evoked. The authors found that depending on the phase of the step cycle, the effect of the visually guided stepping task on corticospinal excitability was either facilitatory or inhibitory, and that this effect was different for the two muscles evaluated (the tibialis anterior, and the gastrocnemius medialis).

A 2001 rapid report by Petersen et al aimed to determine whether increased CSE in humans during walking correlates directly to the activity of spinal motoneurons [30]. Petersen later published a review and discussed how the understanding of human motor control has been impacted by TMS [31].

Another study (2012) compared the effect of assistive and resistive force fields on MEPs in the tibialis anterior (TA) from stimulations delivered exclusively during the swing phase [10]. The experimental protocol is presented below. The assistive or resistive force fields were applied for ten minutes of adaptation. 


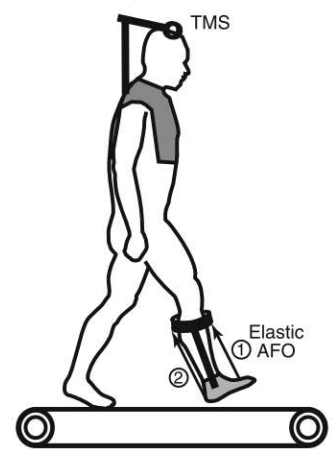

\section{B Experimental protocol}

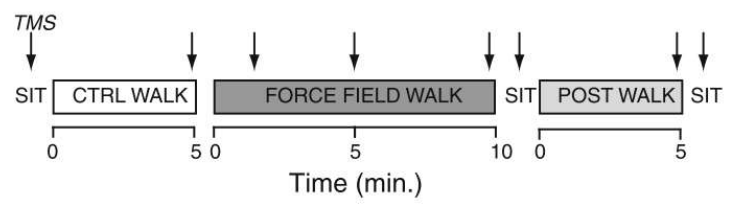

Figure 12: Schematic of experimental set-up (A) and protocol (B) [10].

Using this protocol, Barthèlemy et al divided their MEPs into five groups: control, adaptation 1, 5, and 10 minutes, and recovery. The normalized TA MEP amplitudes were found to be larger when the force field resisted dorsiflexion, and smaller when the force field assisted dorsiflexion. These changes were not present when the MEPs were evoked under the same force fields and with similar muscle activity due to voluntary contraction during sitting. This result suggests that the changes in CSE cannot be solely accounted for by task-demand, i.e. muscle activity. The changes were also present as soon as 1 minute into adaptation, suggesting MEPs adapt quickly to force fields applied while walking.

A locomotor task TMS study has also been conducted with partial and absent body weight support with the use a harness. In their 2013 paper, Knikou et al sought to understand the relationship between ground reaction force and CSE [9]. The muscle activity from control trials was subtracted from MEP magnitude in order to quantify the contribution of the stimulus. This difference was then normalized using the maximum signal, and the subtracted, normalized MEPs were compared across the step cycle. The results showed that the strength of the corticomotoneuronal drive was not altered by partial body weight support as compared to absent body weight support. This result was also replicated in participants across two sessions, task-dependent MEP modulation does not change significantly in the same participant.
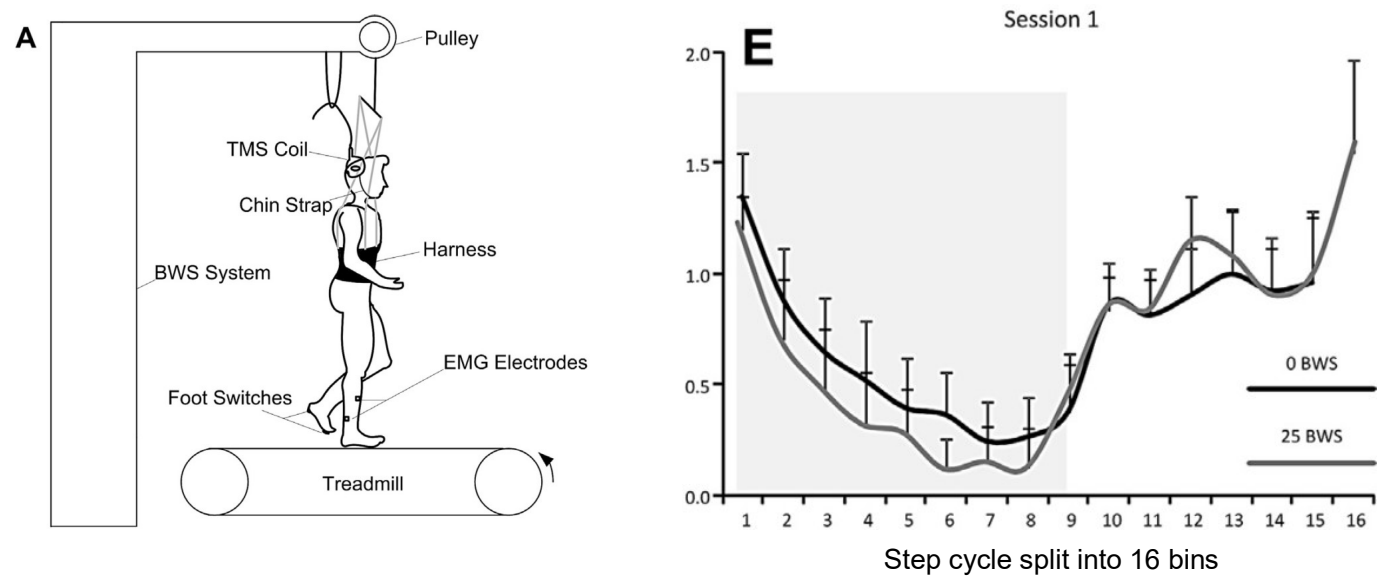

Figure 13: A. The experimental set-up used by Knikou et al [9]. E. The resulting MEP magnitude curves for the tibialis anterior. 
These TMS studies have also been reviewed to provide a wider explanation for the individual phenomena observed. In 2015, Davranche et al conducted a review of TMS studies involving whole body exercise during cognitive tasks, and suggested that while increases in CSE are typically reported, there may also be an accompanying reduction in intracortical inhibition that provides further insight into the mechanisms leading to the increased CSE [32]. This hypothesis is largely fueled by the observed reduction of cortical silent period durations in measured MEPs. The authors suggest that as muscles become more fatigued peripherally, inhibition in the motor cortex is reduced such that cortical control becomes more responsive to inputs, and thus more efficient. Finally, Bestmann and Duque reviewed TMS studies used to decompose action preparation [33]. In this review, Bestmann and Duque concluded the MEPs can provide insight into dynamic aspects of motor preparation during tasks. MEPs are sensitive to control mechanisms such as decision making and high-level cognition (thought), and they also provide a temporal specificity that is otherwise difficult to discern through non-invasive methods.

A brief summary of current signals processing techniques used in biomechanics is provided in the following section.

\subsection{Signals Processing Techniques in Biomechanics}

Different types of sensors have been used in the field of biomechanics to measure experimental variables associated with movement. Commonly used signals in locomotor research are collected from force plates, electromyography (EMG) sensors, and motion capture systems.

\subsubsection{Force Plate Data}

In locomotion, force sensors within larger force plates or instrumented treadmills have been used to detect step onset and offset. For this purpose, force plates work well, as the signal to noise ratio required to detect a step programmatically are high; a participant weighing $50 \mathrm{~kg}$ will produce roughly $500 \mathrm{~N}$ of force when standing, and while the noise will depend on the specific force plate [provide range here], A $50 \mathrm{~N}$ threshold can be used to mark step onsets, with minimal additional quality assurance required.

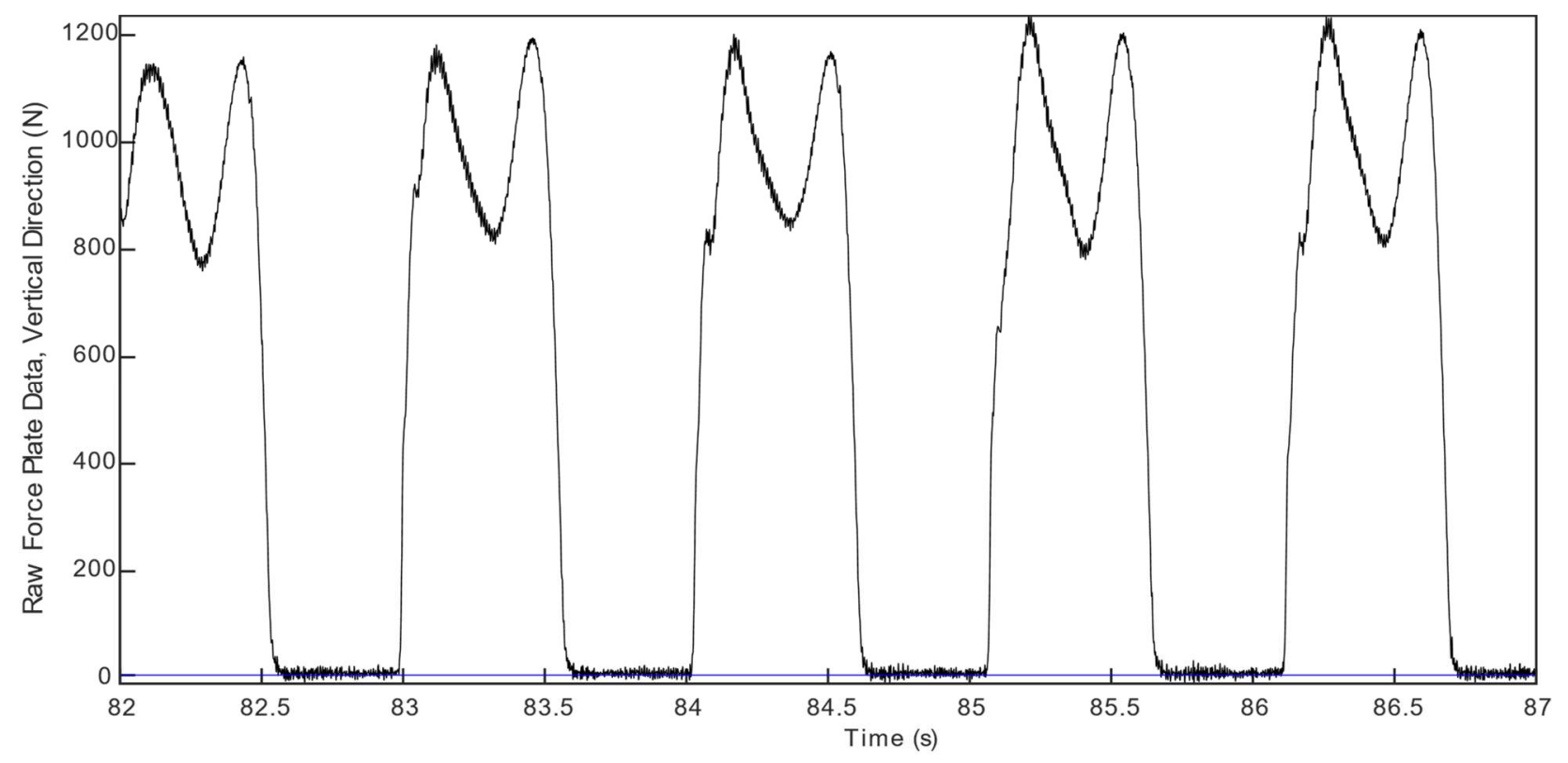

Figure 14: Raw force plate data collected during locomotion, for ground reaction forces in the vertical direction. 
This force plate data can be smoothed and simply thresholded to determine the onset of a step. Once onsets are detected, secondary assessments, such as minimum time between steps during treadmill walking, can be implemented in order to improve the accuracy of the automated step detection.

Force plate data with high resolution has also been used to determine the movement of the center of pressure across the foot during stance. The vertical projection of the center of mass can also be calculated using force and moment data, with results following a sinusoidal pattern similar to the figure shown below.

\subsubsection{EMG Data}

Electromyography (EMG) is used in biomechanics research to measure muscle activity. The noninvasive form of electromyography sensors is comprised of a set of contacts with a ground that measure the electrical potential difference (voltage) across the skin. These measured voltages are the sum of motor unit action potentials, or the current discharged by the motor unit providing stimulation to the muscles to contract. This type of EMG sensor uses multiple references to extract the differentiated signal associated with the propagation of action potential along the sarcolemma and causing contraction of muscle tissue. The recorded signal is mean-zero and noisy, and substantial filtering must occur in order to discriminate regions of muscle activity, or bursts, from non-active states. An example of the resulting EMG data collected from these sensors is plotted in Figure 15.

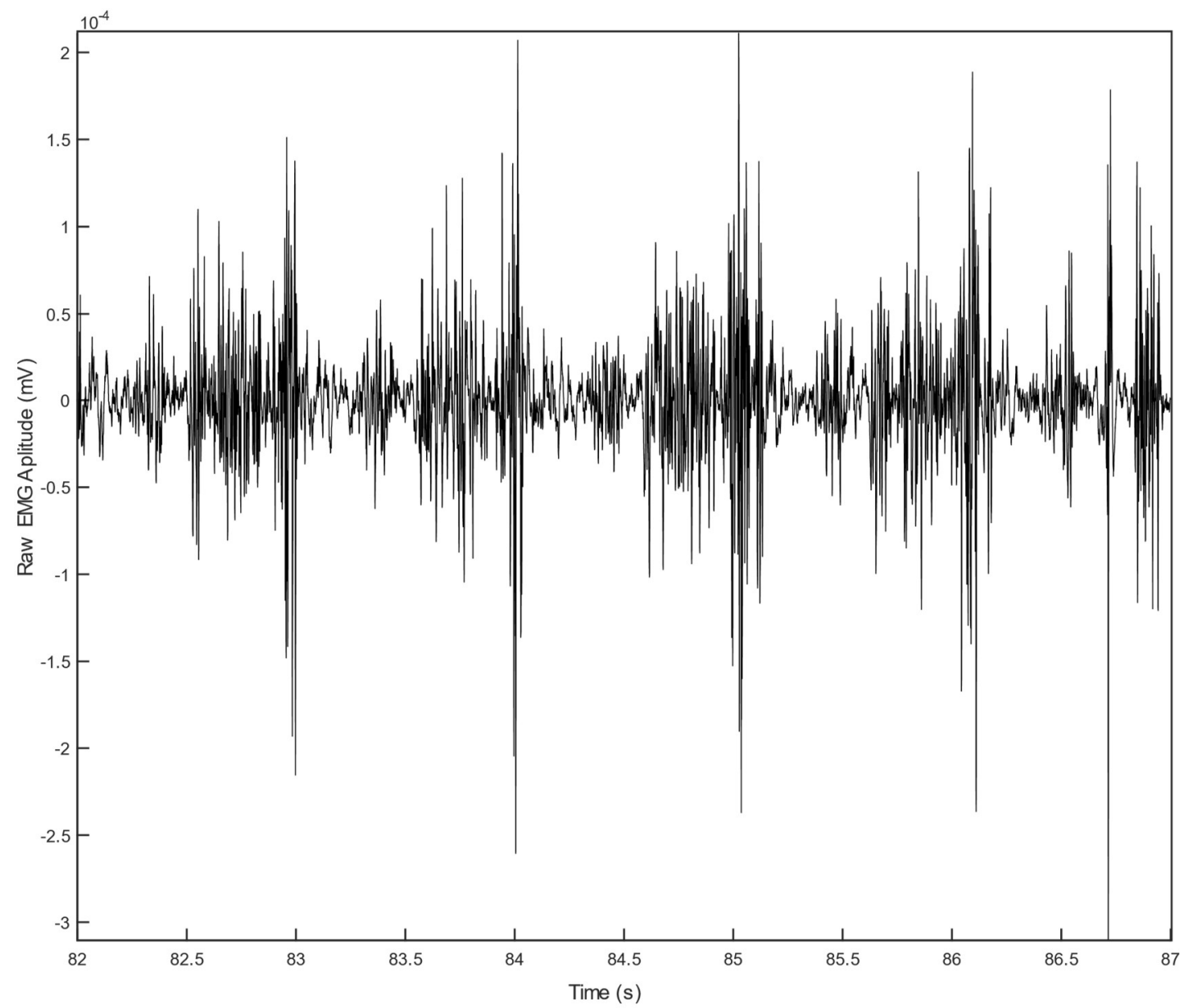

Figure 15: Example of raw EMG data, collected during walking from the right tibialis anterior of a subject. 
The post-processing of EMG signals depends on the variable of interest in the particular study. Usually, EMGs are first high-pass filtered to remove any movement-related biasing and artifacts. Then the signal is rectified and low-pass filtered. The resulting profile is generally related to the recruitment level in the muscle [34].

\subsubsection{Motion Tracking for Neuronavigation}

There are many forms of motion tracking systems used today, with varying levels of involvement for set up or post-processing. The ideal system requires minimal effort on the part of experimenter to set up, and consistently reliable results. For estimating planar angular changes, or relative position between two rigid bodies, tracking arrays of a fixed geometry provide a good compromise to measuring exact position and minimizing set up time and effort.

For TMS studies, the variable of interest is not the absolute position of the coil or the brain, but the relative position of the coil to the head throughout the experiment. Neuronavigation software such as Brainsight, in combination with the hardware required, can be used to monitor this relative position, and display the value in real time. Using additional triggering, the position of the coil relative to the head at the instant of stimulation can also be recorded. This is accomplished by tracking the position and orientation of two fixed geometry tracking arrays, and using two additional tracking arrays for calibration of each array.

The set up required to take advantage of neuronavigation software is simple. Two marker arrays of varying dimensions are programmed into the software (one for the coil, and one for the head). The first tracker is attached to the coil, and the coil is calibrated using a calibration block, which also holds a unique tracking array. This calibration is saved and updated prior to each experiment.

The second array is attached to the head of the participant. A pointer with yet another unique tracking array is used to calibrate the subject tracking array to the head of the subject, such that magnetic resonance imaging (MRI) data can be used to target specific areas of the brain.

\subsubsection{Processing Data for Measurement of Motor Evoked Potentials}

Force plate, EMG, and neuronavigation data are used in combination to calculate response variables used for conducting statistical tests and determining whether effects are significant. The process starts by synchronizing the force plate signals with a common synchronization pulse associated with the triggering of TMS, and the initiation of recording of EMG data. The TMS trigger pulses are read into the same data acquisition system, and are thereby synchronized to the force plate data to within the recording rate of the DAQ, which can be tested by comparing the difference between the trigger input and output, both of which are streamed into the DAQ. To synchronize the EMG data, a pulse is sent from the EMG data acquisition system (DAQ) to the force plate DAQ, and this start pulse is used to measure the delay between the force plate and TMS pulse signals, and the EMG system.

The first signal processed is the force in the vertical direction $(\mathrm{Fz})$ from the force plates under the treadmill belts. This data was smoothed and thresholded to $50 \mathrm{~N}$ to identify step onsets. Next the TMS pulses are identified by thresholding the signal. Using these pulses, the step cycles are separated into control steps, those without stimulation, and stimulation steps, those containing a stimulation within the duration of the step cycle. 
Next, the difference between the time of step onset and TMS pulse is calculated for each TMS pulse. This time is divided by the duration of that step to determine the percentage of step cycle when the stimulation was administered. This percentage can be used to group MEPs occurring due to stimulations in the same phases of the step cycle in statistical analysis.

Processing of EMG data to create response variables for conducting statistical tests can be arduous. The features of interest are typically the MEP latency, MEP peak-to-peak amplitude, the integrated MEP area, and the length of the cortical silent period (CSP). Many techniques have been applied to EMG data to pull out, including brute-force manual identification by human operators. Two specific methodologies have been tested in this work, and are described in further detail in Chapter 4. 


\section{CHAPTER 3: EVALUATING CORTICOSPINAL EXCITABILITY DURING LOCOMOTION}

\subsection{Introduction}

This chapter describes the main contribution of this work: the TMS experiment conducted to evaluate corticospinal excitability during locomotion in healthy adult humans. Detailed descriptions of the methods and equipment used in this experiment are provided in the Background section.

The purpose of this experiment was to test the hypothesis that limb velocity has an effect on corticospinal excitability in healthy humans, during normal walking tasks.

\subsection{Methods}

Fifteen subjects were recruited to walk on a split-belt, force-plate instrumented Bertec treadmill. Subjects were instrumented with the Delsys Trigno wireless EMG sensors, and wore a harness that was secured to a rigid frame to remove the possibility of participants tripping or otherwise falling onto the treadmill during the experiment. There were four conditions for each participant, with a randomly generated schedule for each participant. Subjects walked with either symmetrical velocity conditions (1 $\mathrm{m} / \mathrm{s}$ or $1.25 \mathrm{~m} / \mathrm{s}$ for both legs), or asymmetrical velocity conditions $(1 \mathrm{~m} / \mathrm{s}$ and $1.25 \mathrm{~m} / \mathrm{s}$ for left leg and right leg, respectively, or vice versa). Prior to stimulation during each trial, each participant took ten steps to establish an average step time for stimulation scheduling purposes. Two incomplete datasets were excluded due to errors in data collection, and one participant was excluded due to irregularities in their gait patterns.

\subsubsection{Experimental Design \& Factor Selection}

The experimental design of this study tested the representation of limb velocity within descending motor commands, as measured indirectly through CSE. The experiment was designed to yield comparisons for two factors: left and right limb velocity, with a test available for interaction between the two velocities. Each limb had two slow velocity $(1 \mathrm{~m} / \mathrm{s})$ trials, and two fast velocity $(1.25 \mathrm{~m} / \mathrm{s})$ condition trials. Likewise, there were two symmetrical velocity trials, and two asymmetrical velocity trials. This design ensures adequate data to indicate whether trends exist in the behavior of corticospinal excitability according to these factors.

The final velocity conditions selected are given in Table 1. These belt speeds were selected to be comfortable, but still provide enough of a difference to induce a detectable change in CSE.

Table 1: Velocity conditions with left and right belt speeds defined.

\begin{tabular}{c|cc}
$\begin{array}{c}\text { Velocity } \\
\text { Condition }\end{array}$ & $\begin{array}{c}\text { Left Belt Speed } \\
(\mathrm{m} / \mathrm{s})\end{array}$ & $\begin{array}{c}\text { Right Belt Speed } \\
(\mathrm{m} / \mathrm{s})\end{array}$ \\
\hline 1 & 1 & 1 \\
2 & 1.25 & 1.25 \\
3 & 1 & 1.25 \\
4 & 1.25 & 1
\end{tabular}

Each experiment lasted two hours including consenting, set-up, and data collection. 
A National Instruments (NI) DAQ was used to collect force data from the Bertec instrumented treadmill, as well as a sync pulse from the EMG wireless DAQ $(1 \mathrm{kHz})$. EMG signals were collected with the Delsys Trigno wireless EMG system $(2 \mathrm{kHz})$.

\subsubsection{Split-belt Treadmill}

The split-belt treadmill used for this experiment was a Bertec force plate instrumented treadmill. The force plate data was collected at $1000 \mathrm{~Hz}$. This data was used to detect onset events for separating data into steps post experiment, and to arm the TMS machine for a scheduled stimulation in real-time during each experiment. Due to the regularity and low signal to noise ratio of this signal, the force in the upwards direction was window averaged to 25 samples (or $25 \mathrm{~ms}$ of data), and simply thresholded using a $50 \mathrm{~N}$ limit to detect when a participant had begun loading one side of the treadmill and begun stance phase on that limb.

Subsequent onset events on the same side were used to define step cycles throughout the trial. Using this method, the force data was separated into individual onset to onset events to produce average ground reaction force profiles for all subjects. Sufficiently irregular gait patterns (from other participants) were a criterion for excluding participant data, however all participants exhibited regular gait patterns during control steps based on the force plate data. One participant was excluded due to irregularities in their gait patterns.

The use of a split-belt treadmill enabled the implementation of asymmetric velocity conditions described in 3.2.1. The velocity condition and delay schedules for each experiment was randomly generated during initialization for each experiment during set-up, and the final schedules for all participants is presented in Table 2 below.

Table 2: Velocity condition schedule for all subjects. Conditions are defined in Table 1.

S01 S02 S03 S04 S05 S06 S07 S08 S09 S10 S11 S12 S13 S14 S15

\begin{tabular}{|l|l|l|l|l|l|l|l|l|l|l|l|l|l|l|l|}
\hline Trial 1 & 3 & 3 & 1 & 3 & 2 & 3 & 3 & 2 & 2 & 1 & 1 & 2 & 4 & 1 & 3 \\
\hline Trial 2 & 1 & 1 & 3 & 1 & 1 & 1 & 1 & 1 & 1 & 3 & 3 & 4 & 1 & 3 & 1 \\
\hline Trial 3 & 2 & 2 & 2 & 2 & 4 & 2 & 2 & 4 & 3 & 2 & 2 & 1 & 2 & 2 & 2 \\
\hline Trial 4 & 4 & 4 & 4 & 4 & 3 & 4 & 4 & 3 & 4 & 4 & 4 & 3 & 3 & 4 & 4 \\
\hline
\end{tabular}

\subsubsection{EMG Sensors}

Electromyography (EMG) sensors were used to collect muscle activity signals. EMG data was recorded from 10 muscles in the right leg, and 5 muscles in the left leg using wireless surface electrodes, aligned with the muscle fibers of interest. The muscles for which muscle activity data was collected are listed in Table 3 below. 
Table 3: Legend for muscles instrumented with EMG sensors for each participant.

\begin{tabular}{|r|l|r|l|}
\hline \multicolumn{2}{|c|}{ Muscles Observed in Both Legs } & \multicolumn{2}{c|}{ Muscles Observed in Right Leg Only } \\
\hline TA & Tibialis Anterior & SOL & Soleus \\
\hline GASL & Gastrocnemius Lateralis & VASL & Vastus Lateralis \\
\hline GASM & Gastrocnemius Medialis & VASM & Vastus Medialis \\
\hline BICF & Biceps Femoris & SART & Sartorius \\
\hline RECF & Rectus Femoris & SMTD & Semitendinosus \\
\hline
\end{tabular}

The area below each sensor was shaved and cleaned with an alcohol swab to reduce noise and ensure good adhesion of the sensor pad. Raw EMG signals were demeaned and stored in a MATLAB database for post processing. The force plate data was marked for onsets, and TMS pulse data was used to identify step cycles during which a stimulation occurred.

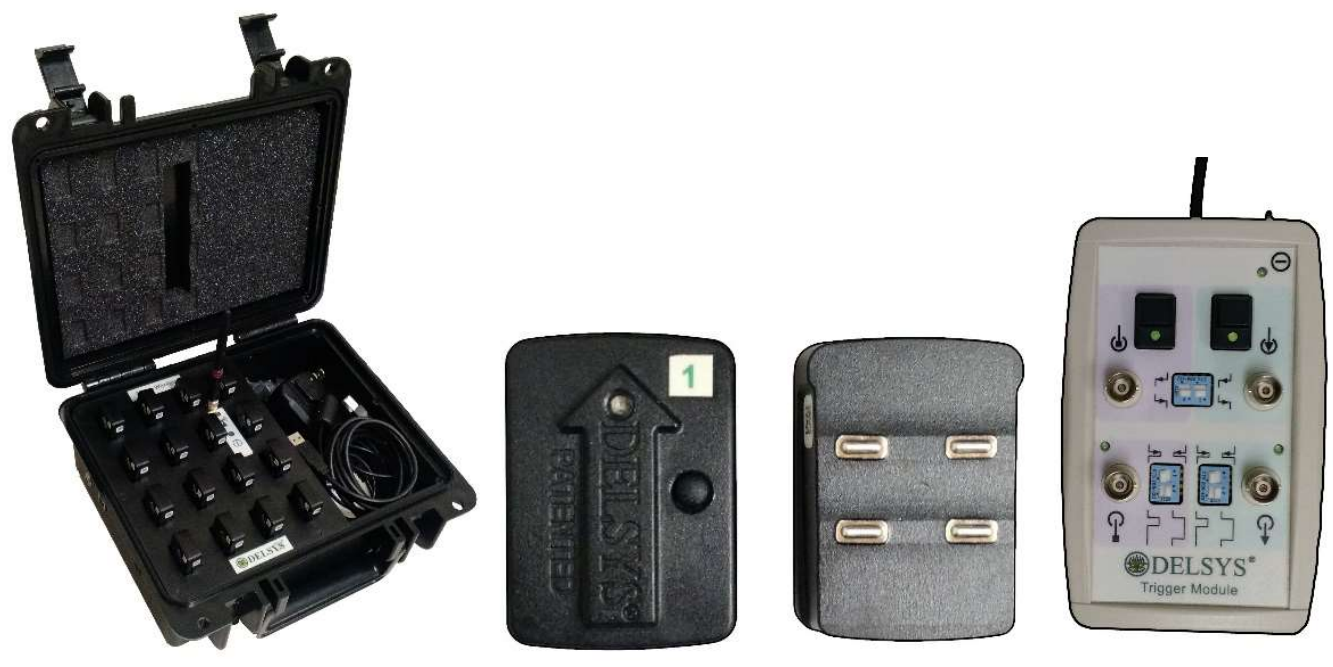

Figure 16: The Delsys Trigno hardware.

EMGs were used to identify regions of muscle bursts for each muscle using the supervised adaptive threshold crossing method developed by by Yakovenko et al [35]. The onsets and offsets of each active phase in EMG signals was used to segment the muscles into two distinct muscle groups, flexors and extensors, for additional statistical testing.

\subsubsection{Transcranial Magnetic Stimulation}

A Magstim $200^{2}$ stimulator was used to deliver pulses through a $110 \mathrm{~mm}, 1^{\text {st }}$ generation double cone coil, also from Magstim. The geometry of this coil is such that current can be induced in the cortical area associated with the lower limbs. 


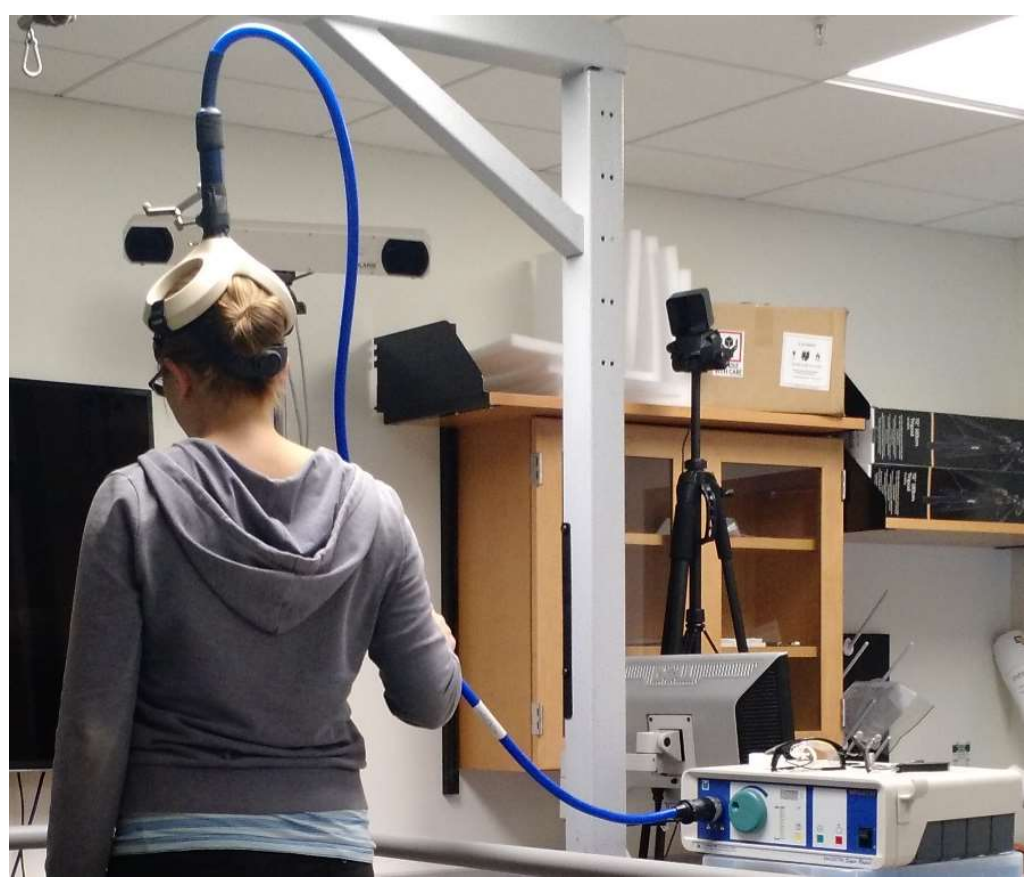

Figure 17: The Magstim double cone coil positioned over the vertex.

Single pulse TMS was used to evoke responses, or motor evoked potentials (MEPs) in the leg muscles of each participant. The stimulations were externally triggered by custom MATLAB software through an analog output port in the National Instruments data acquisition system. The stimulations were spaced with at least $5 \mathrm{~s}$ intervals according to the diagram below. This type of stimulation corresponds to single-pulse TMS, which is generally excitatory to the M1. Repeated stimulations with shorter than $5 \mathrm{~s}$ intervals correspond to repetitive TMS, which is generally inhibitory to the M1. The implementation was completed using two flags: one to ensure five seconds have passed since the previous stimulation, and another to send the stimulation pulse following the scheduled delay of time after right leg step onset. The estimated average step cycle time for the right leg, calculated prior to beginning stimulation, was used to initialize the delay schedule. A trigger for stimulation was sent only when both these flags were true.

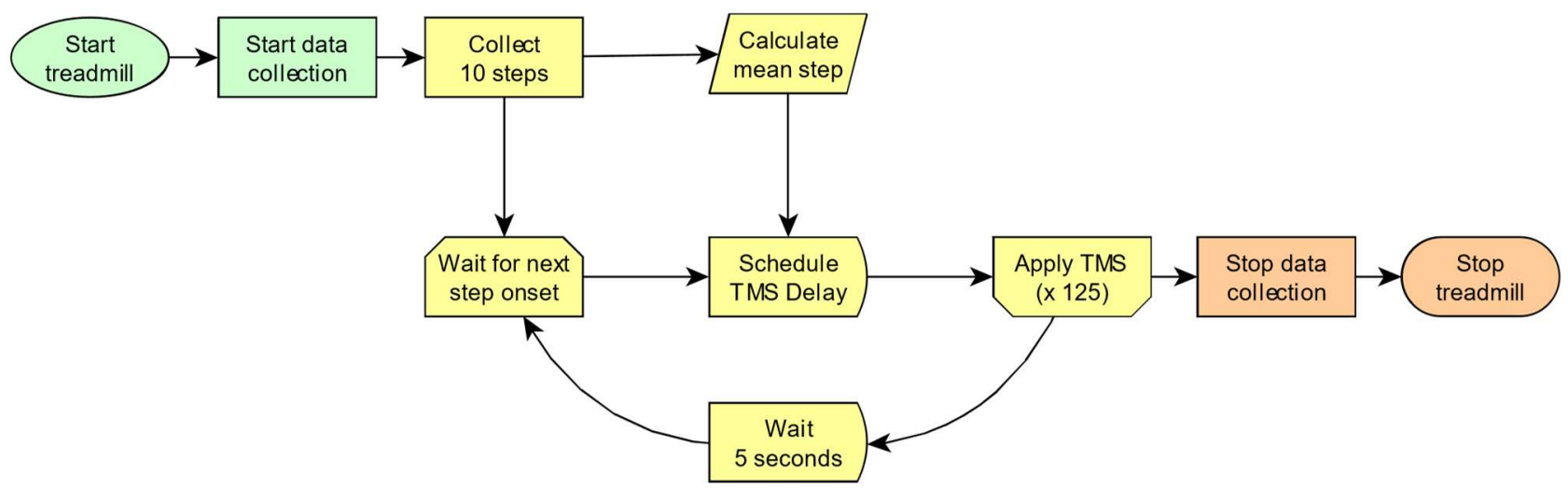

Figure 18: A flowchart of the experimental triggering protocol.

In addition to triggering the stimulation, the NI DAQ was also used to collect a signal output from the Magstim stimulator. The stimulator was set up to output a high signal (0-5 v) of $750 \mathrm{~ms}$ at the time 
of each stimulation. The leading edge of this signal was used define the stimulation time for each MEP, and to identify stimulation and control steps for analysis.

\subsubsection{Neuronavigation}

Brainsight hardware and accompanying software was used in order to determine the relative position of the coil to each participant's head in real time, and save this data at the time of each stimulation. A tracking array was affixed to the TMS coil. A collar was designed, 3d printed, and attached to the Magstim coil to depress the stimulation button and allow for external triggering. This collar had a position to affix the coil tracking array provided with the Brainsight system. A second array for subject tracking was attached to the face of each participant, on the right cheekbone and below the right eye.
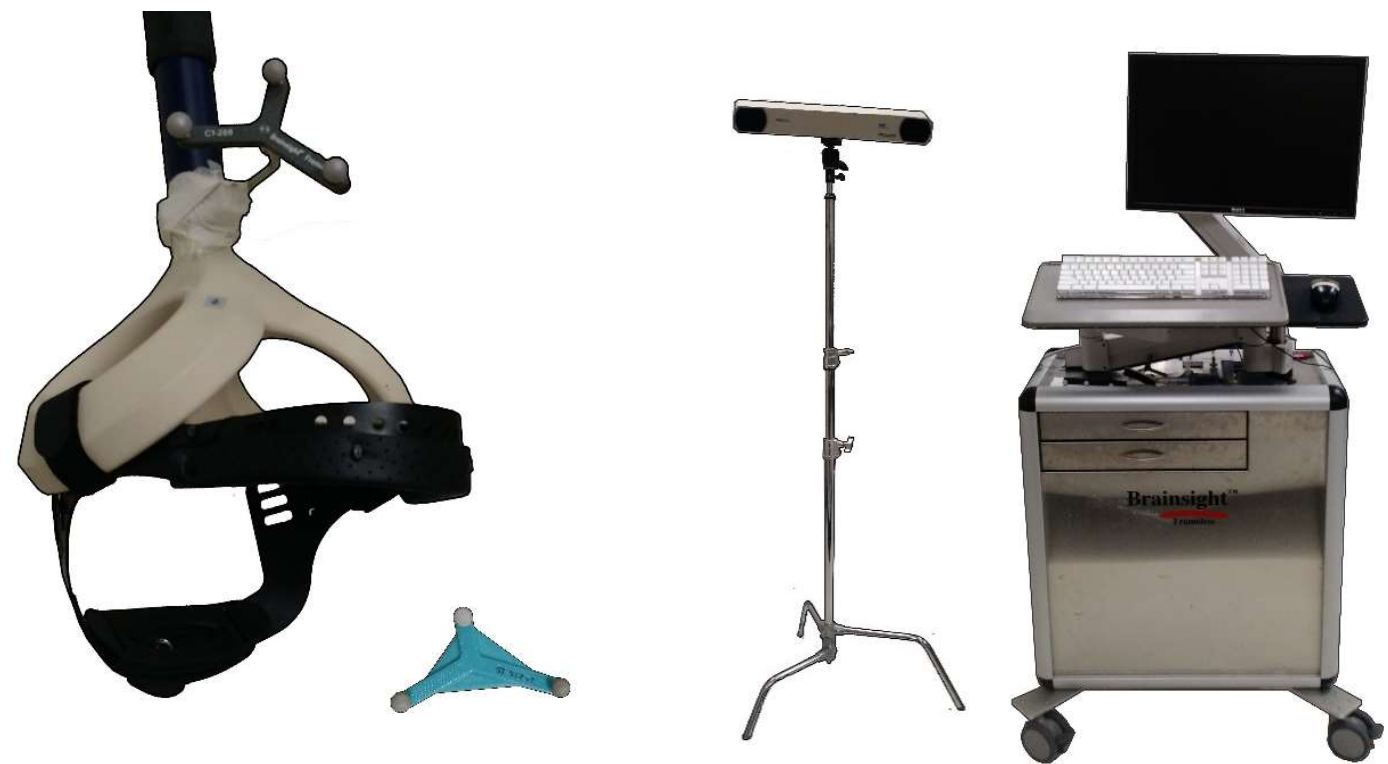

Figure 19: The hardware used for neuronavigation. On left, the subject array and coil array, affixed to the coil. On right, the Polaris camera and Rogue Research/Brainsight computer station.

These arrays were used in conjunction with a Polaris motion tracking camera set up to the right of the participant, and the camera was placed such that marker drop out was eliminated. Prior to the start of each series of experiments, the TMS coil was calibrated to the tracking array using the Brainsight system calibration block.

A previously collected magnetic resonance image (MRI) scan of a human brain was used for targeting purposes. Four locations easily identifiable points on the scan were defined in the software for calibration purposes, and are shown in Figure 20. This scan was calibrated to the size and proportion of each subject by bringing a pointer array to each point defined in the scan. The process described automatically scales the scan to the subject's head, and combined with the coil calibration, will display the approximate region of stimulation of the coil throughout the experiment. As long as the coil and subject tracking arrays remain in the same position, an identified target location can be consistently stimulated with high accuracy.

The average target error during experiments is presented in the results. 


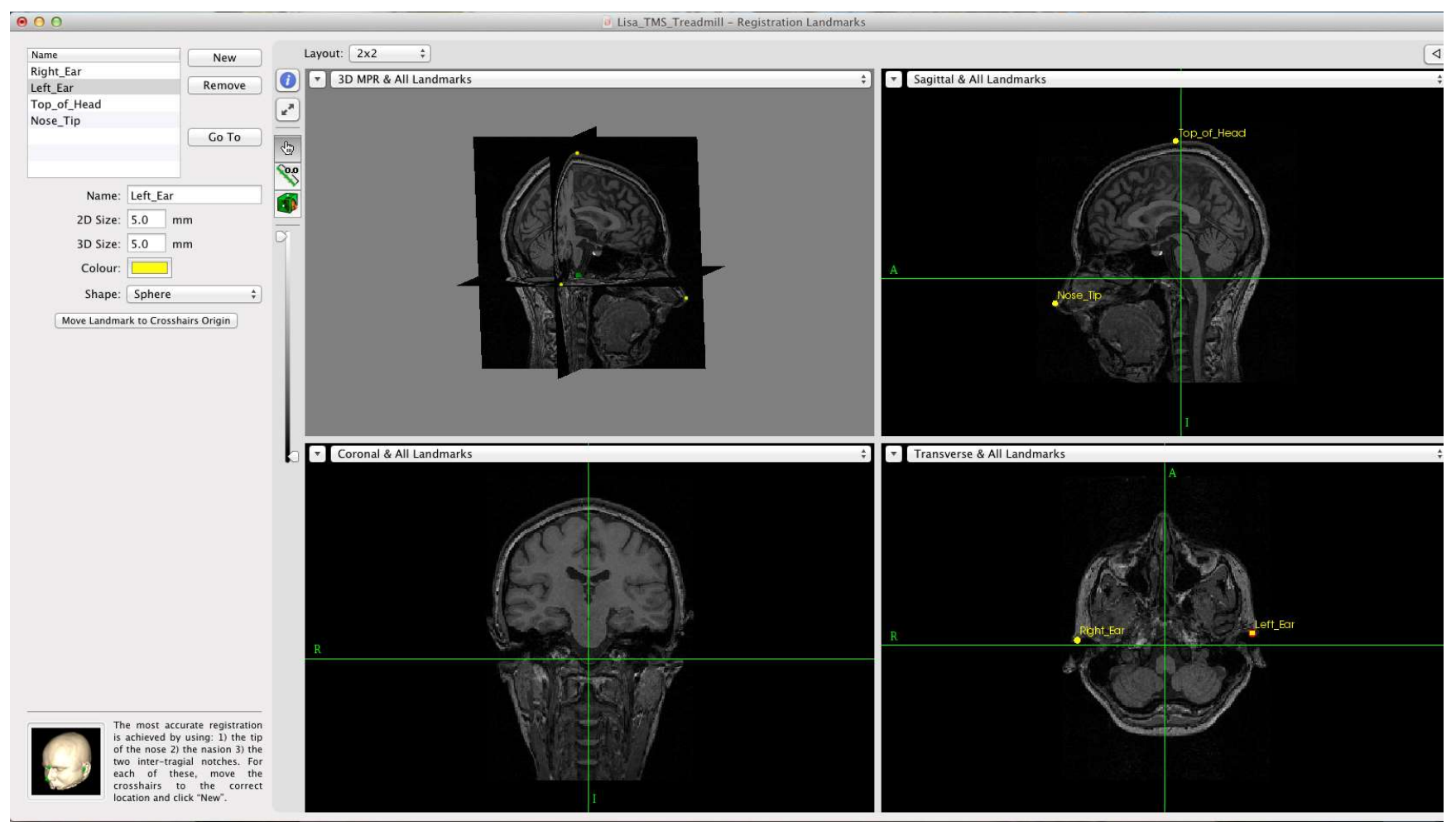

Figure 20: The locations used to locate the subject tracker to the MRI scan.

\subsubsection{Experiment triggering}

TMS pulses delivered from a Magstim stimulator at the time of each pulse. Step onset and offsets were detected in real time by thresholding incoming packets of force data. A threshold of $50 \mathrm{~N}$ was used to ensure noise was not counted as a step.

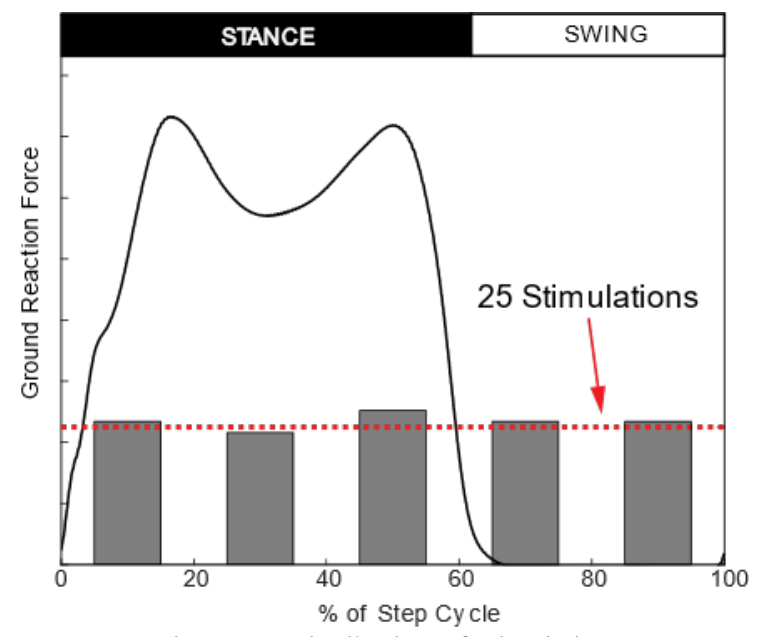

Figure 21: Distribution of Stimulations.

An example of the distribution of stimulations throughout the step cycle for one participant, for one trial. With 125 stimulations, there are roughly 25 stimulations in each $20 \%$ of the step cycle. These constitute the bins that are used for analysis, since each stimulation will not occur at exactly in the middle of the bin. 
All collected data, with the exception of neuronavigation software output, was imported into the custom MATLAB database software, Boxsci. Custom MATLAB scripts were used to identify step onset events using force plate signals, to allow for segmentation and time-normalization of the EMG and force plate data. The TMS synchronization pulse was also used to identify stimulation time and select the appropriate time window for MEP quantification, which is described in further detail below.

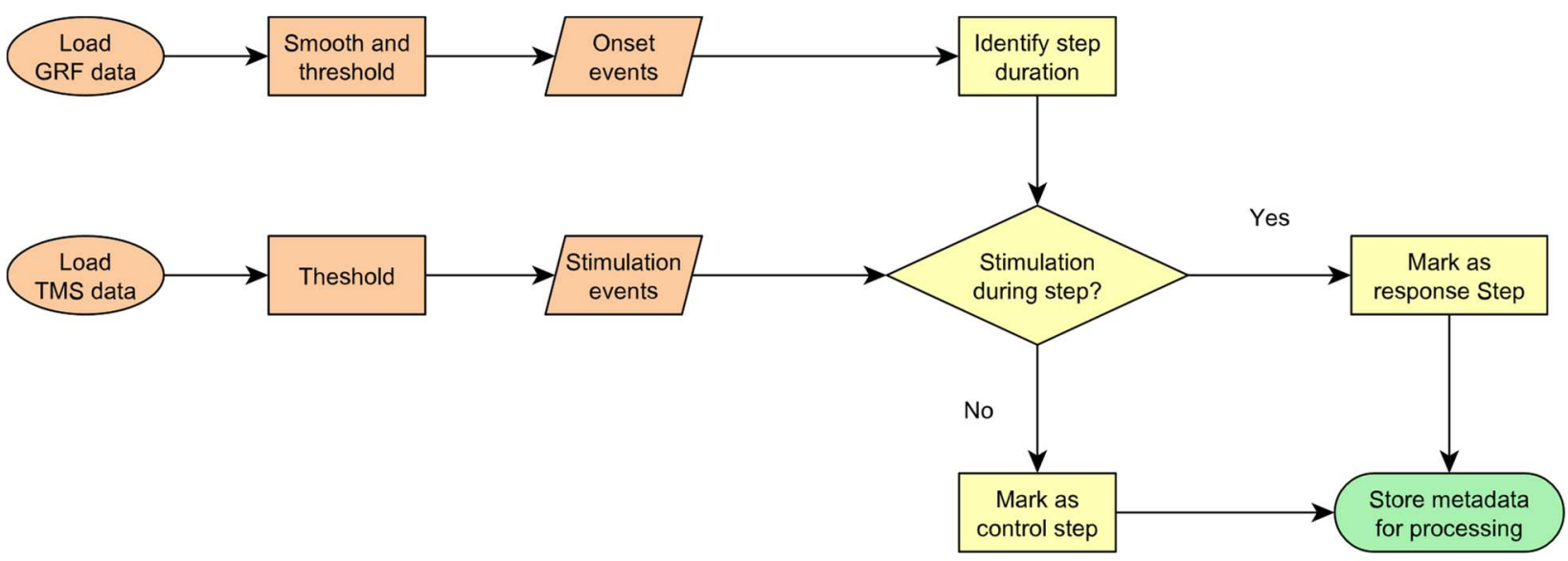

Figure 22: A flowchart describing part of the data processing pipeline, used to generate and save metadata identifying step onset and stimulation events, and separating control steps.

\subsubsection{Quantifying MEP magnitude}

The data collected was processed to measure the response variables of interest. For this analysis, the response variables include the peak to peak magnitude, the integrated area, and the duration of the cortical silent period for each MEP. Additionally, the root-mean-square muscle activity directly prior to each stimulation was saved in order to normalize MEP peak to peak magnitude.

The peak-to-peak value of each MEP was calculated by subtracting the minimum value in each 75 $\mathrm{ms}$ following stimulation from the maximum value in that same time period. This period was selected by inspection of when MEP peak amplitudes were occurring for most participants.

The integrated area of the MEP was also used to quantify MEP magnitude, however this analysis has not been presented herein. The onset of MEP was different between participants according to their height, as dictated by the conduction velocity and length of pathways from the M1 to each muscle. A period of time that contained the entirety of most MEPs as determined from average MEP signals for each participant was used to define the area of interest. The duration of each period was kept constant for this analysis $(40 \mathrm{~ms})$. Integrated control step EMG was used to normalize the corresponding integrated MEP areas for that muscle.

Both methods are illustrated in Figure 23. 


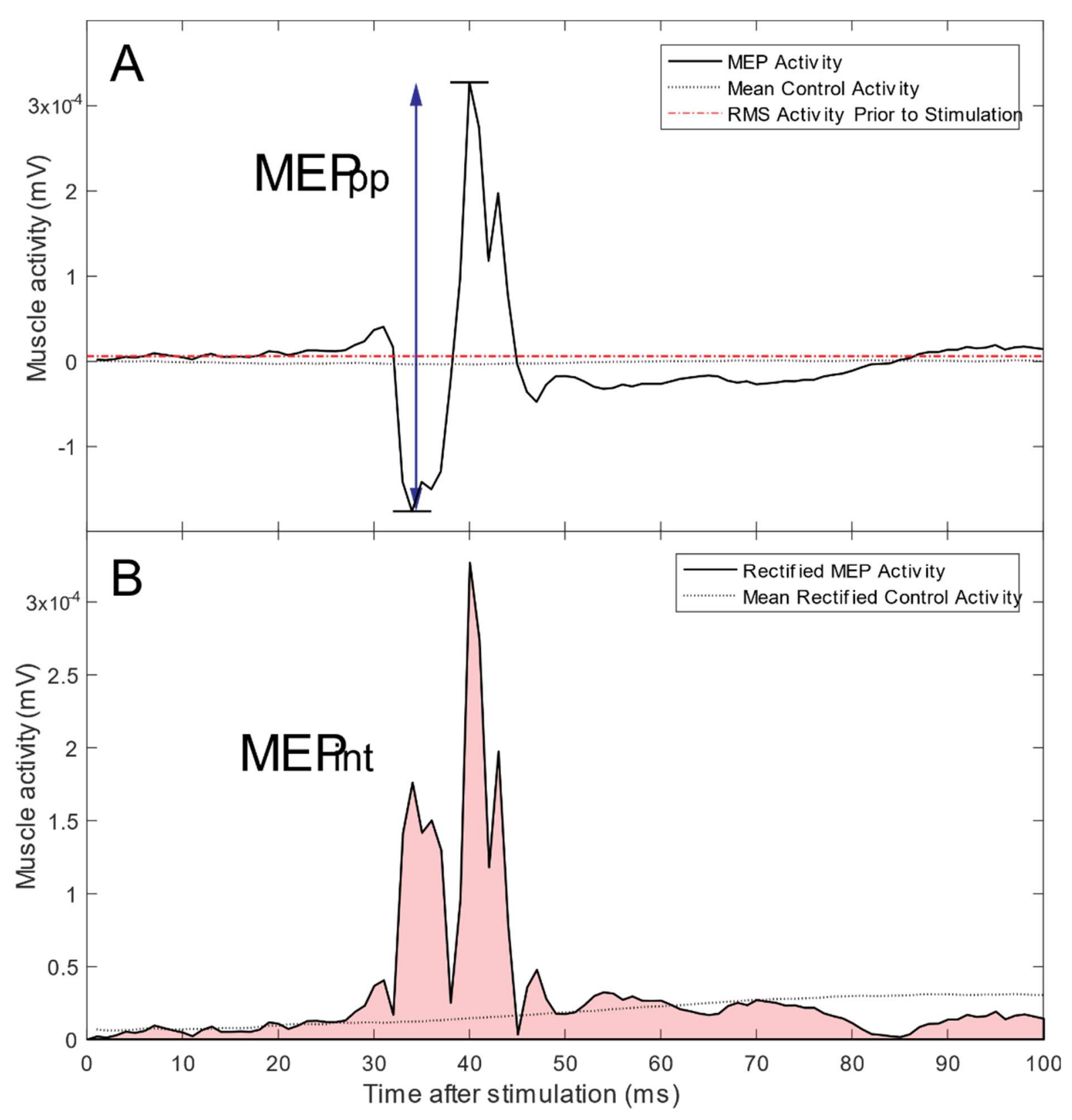

Figure 23: MEP processing demonstrated for a single evoked response.

MEPs were excluded according to the following criteria, adapted from van de Ruit et al [36].

1. (Remove noisy data) Calculate RMS of EMG prior to stimulation. Calculate mean of all MEP peak to peak values for that trial. Exclude that stimulus if the background RMS is within two standard deviations of the mean of all MEP peak to peak values.

2. (Remove outliers) Calculate MEP peak to peak values for all stimuli in trial. Calculate mean of all MEPpp values in trial. If a single MEPpp value is not within mean +/- two standard deviations of all MEPpp values in that trial, exclude the MEP. 


\subsubsection{Statistical Analysis}

The result of the post-processing is a flat table containing factors and response variables for each MEP in each muscle signal measured, in each participant, for every trial. This is a total of at least $125 * 4 * 15 * 15=112500$ MEPs for the data set, since additional stimulations were provided when technical difficulties were encountered to ensure roughly 20 MEPs, or repetitions, were available for statistical tests for each bin, for each condition. Three participants were excluded due to technical implementation during data collection - the rate at which muscle activity data was collected was different from other trials.

Difference between means of response variables from different conditions were tested for significance using multiway analysis of variance (ANOVA). 


\subsection{Results}

The remaining participant data ( $\mathrm{n}=12$ participants, 6 male, 6 female, mean 24.9 years of age) was included in the final analysis. In this section, ground force reaction profiles are used to discuss the difference between the locomotor tasks. Filtered, rectified, muscle activity signals are also provided for discussion. Muscle burst summaries are provided and the grouping of muscles into extensors and flexors is provided. Finally, the results of statistical tests are presented.

\subsubsection{Gait Patterns}

All participants exhibited similar gait patterns during control steps. Although individual steps varied, the average control steps for each participant were similar to those of other participants when forcenormalized to participant mass, and time-normalized to step cycle. The mean, time-normalized ground reaction profile for all participants is presented in Figure 24.
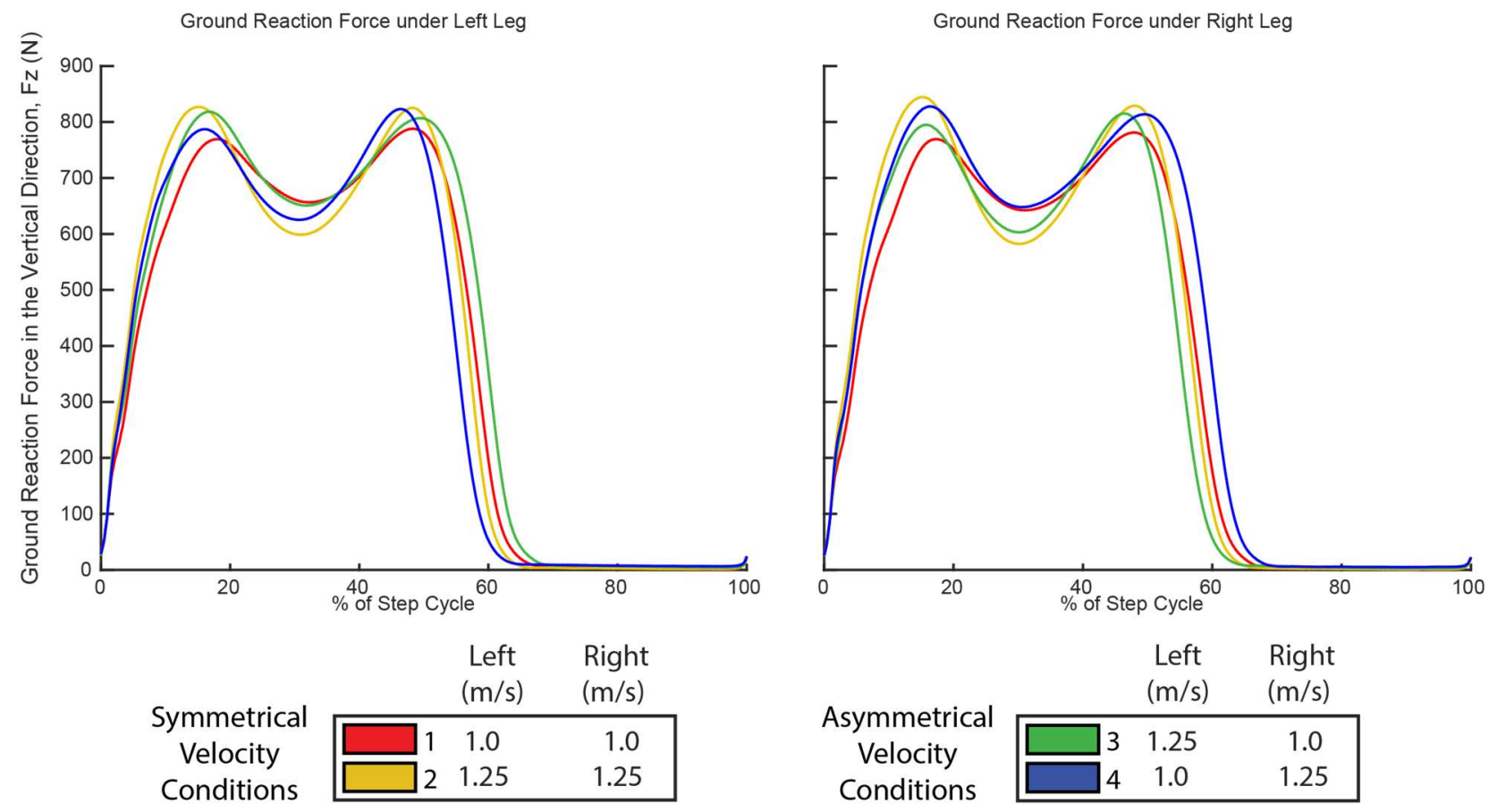

Figure 24: Example ground reaction profiles for a single subject.

The velocity of the limb most greatly affects the peak ground reaction force on weight acceptance during the first stage of stance. The push off force is more stable across velocity conditions. For conditions in which the limb is moving faster, the difference between maximum ground reaction force the valley during stance is greater than it is when the limb is moving slower, whether the overall condition is symmetrical or asymmetrical.

The percentage of the step cycle spent in stance also changes according to the velocity condition. For the symmetrical conditions, the percentage spent in stance for the faster condition is slightly less [quantify], however it is roughly as expected at about $60 \%$ of the step cycle. For asymmetrical conditions, the stance percentage for each limb is greatest when it is the faster limb. That is, the left leg will spend a greater proportion of the step cycle in contact with the ground when the left belt velocity is greater than the right belt velocity, and vice versa. 
The muscle activity scales in time in the same manner as the gait patterns; that is, according to whether the limb was in the stance or swing phase of the step cycle. Mean EMG traces normalized to maximum EMG for control steps taken by all participants are shown in Figure 25. The magnitude of the muscle activity did not have patterns that were as clear, however for some velocity conditions, muscle bursts present in the other conditions disappeared.
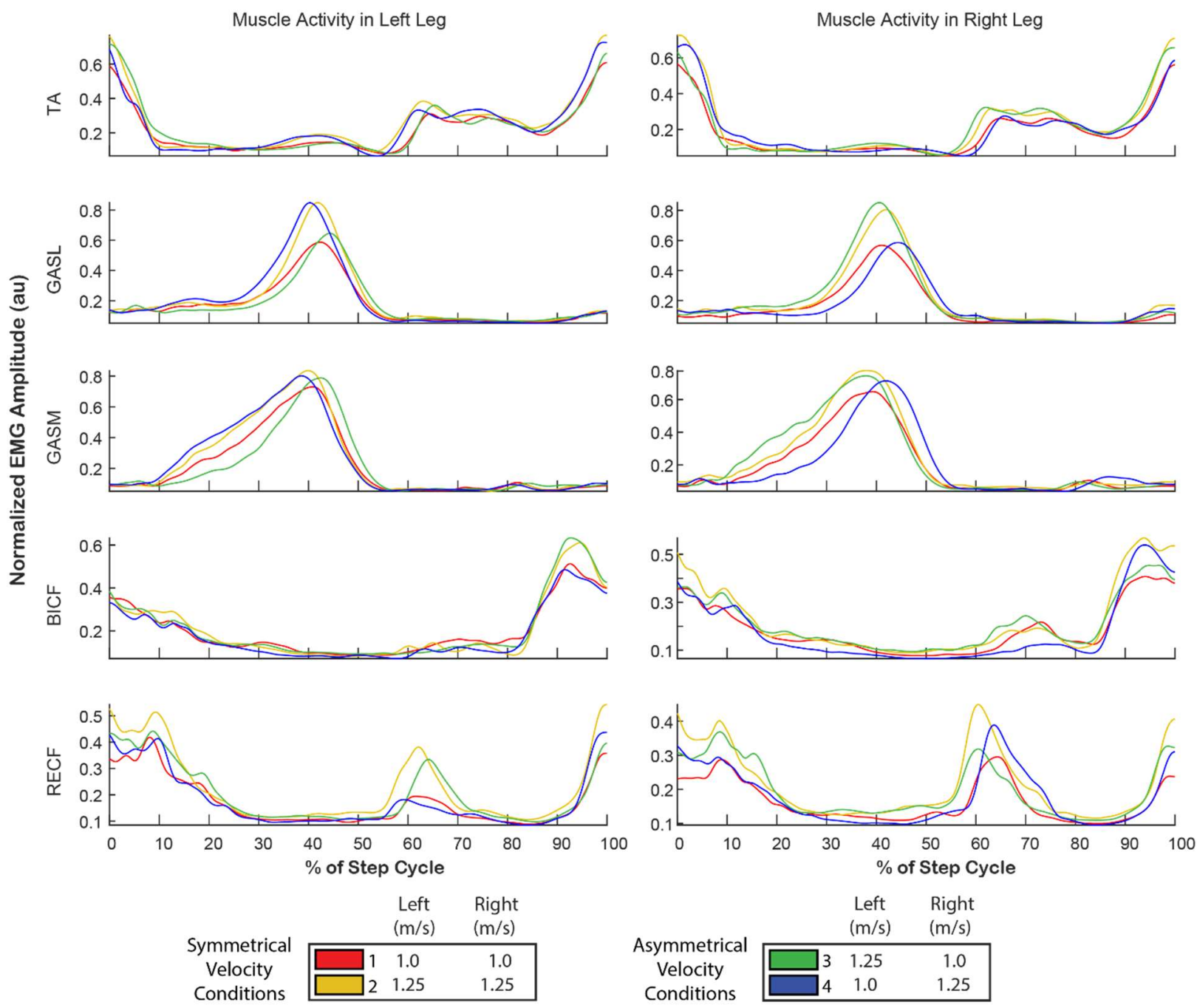

Figure 25: Mean muscle activity during control steps, normalized to maximum EMG collected during control steps in each muscle.

There is a symmetricity in the behavior of the muscles of the left and right legs according to the condition. For example, there was a larger peak activity at the end of swing when the left belt was moving faster, either as part of a symmetrical or asymmetrical belt condition. This behavior was also evident for the right leg, albeit for the opposite asymmetrical condition. Likewise, the activity of the asymmetrical condition in which the belts were moving slower is more similar to the slow symmetrical condition for muscles in both legs.

Among the common muscles (those measured on both legs), the tibialis anterior was always active during swing, with a peak activity at the end of swing and beginning of stance. The gastrocnemius lateralis and medialis were most active during stance, with peak activity at toe off. The biceps femoris and rectus 
femoris were active at the start of stance, and during swing to varying degrees. The peak activity for both muscles was toward the end of swing and into early stance.

The filtered muscle activity was used with a burst detection algorithm to identify primary bursts of activity for each muscle. This was used to separate the muscles into flexors, or muscles most active during swing, and extensors, or muscles most active during stance.

\subsubsection{Muscle Groups - Flexors and Extensors}

The following diagram, Figure 26, illustrates the division of muscles into flexors and extensors as dictated by onset and offset of primary muscle bursts for each muscle.

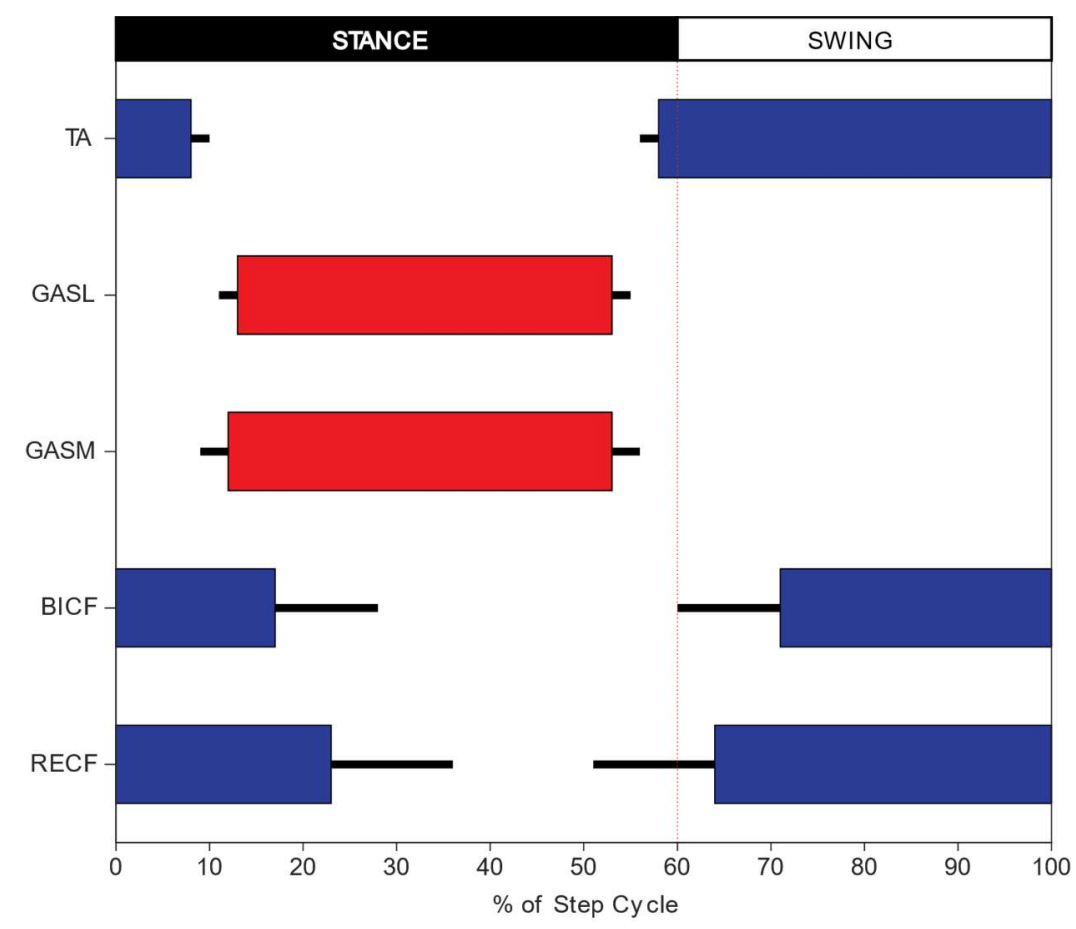

Figure 26: Cross subject $(n=12)$ primary muscle bursts for muscle activity in the left leg.

This figure shows mean and standard deviation of burst regions for muscle activity.

The muscles within the flexor group used for analysis, which contained the muscles most active during swing, were the TA, SART, SMTD, BICF, and RECF. The muscles within the extensor group used for analysis, which was most active during stance, were the SOL, GASL, GASM, VASL, and VASM.

\subsubsection{Neuronavigation with target tracking}

The output of the Brainsight neuronavigation software from Rogue Research was evaluated to determine the consistency of stimulation for each trial. Overall, the precision of the location of stimulation provided by the support technique was within $2 \mathrm{~mm}$, and the average error across all trials and stimulations was $3 \mathrm{~mm}$ as presented in Table 4. The error for each stimulation was the distance from each stimulation location (at least 500 per participant) to the stimulation target area identified and saved during experimental set-up. The average angular error was 2.3 degrees, with a standard deviation of 1.2 degrees. Note that the technique helped apply the stimulations most precisely for the slow, symmetrical condition. The utility of this method also improved with the number of participants. 
A screenshot of the stimulation locations superimposed on the MRI data used for neuronavigation is provided in Figure 27, using the locations saved during a session for a single participant. This illustrates the consistency in stimulation location achieved across four trials with breaks in between, comprising data collection for the four velocity conditions.

Table 4: A summary of the average target and angular errors for each session. Data for subjects S02-S04 was not recorded.

\begin{tabular}{r|rc|cc}
$\begin{array}{r}\text { Subject } \\
(\mathbf{n}=\mathbf{1 0})\end{array}$ & \multicolumn{2}{|c|}{$\begin{array}{c}\text { Target Error } \\
(\boldsymbol{\mu} \pm \boldsymbol{\mathbf { m m }})\end{array}$} & \multicolumn{2}{|c}{$\begin{array}{c}\text { Angular } \\
\text { Error }\left(\boldsymbol{\mu} \pm \boldsymbol{\sigma}^{\circ}\right)\end{array}$} \\
S01 & 2.4 & \pm 1.8 & 2.4 & \pm 1.0 \\
S05 & 3.0 & \pm 2.4 & 4.9 & \pm 1.6 \\
S06 & 4.6 & \pm 3.1 & 3.6 & \pm 1.8 \\
S07 & 3.6 & \pm 3.5 & 2.1 & \pm 1.9 \\
S08 & 3.5 & \pm 2.1 & 2.5 & \pm 1.2 \\
S09 & 2.7 & \pm 1.6 & 2.1 & \pm 0.8 \\
S10 & 3.1 & \pm 1.1 & 1.2 & \pm 0.7 \\
S11 & 2.8 & \pm 1.4 & 1.8 & \pm 1.0 \\
S12 & 3.8 & \pm 2.7 & 2.4 & \pm 1.5 \\
S13 & 1.9 & \pm 1.2 & 1.2 & \pm 0.8 \\
S14 & 2.2 & \pm 1.4 & 1.2 & \pm 0.8 \\
S15 & 2.4 & \pm 1.6 & 2.5 & \pm 0.9 \\
\hline Summary & $\mathbf{3 . 0}$ & $\mathbf{\pm 2 . 0}$ & $\mathbf{2 . 3}$ & $\mathbf{\pm 1 . 2}$
\end{tabular}
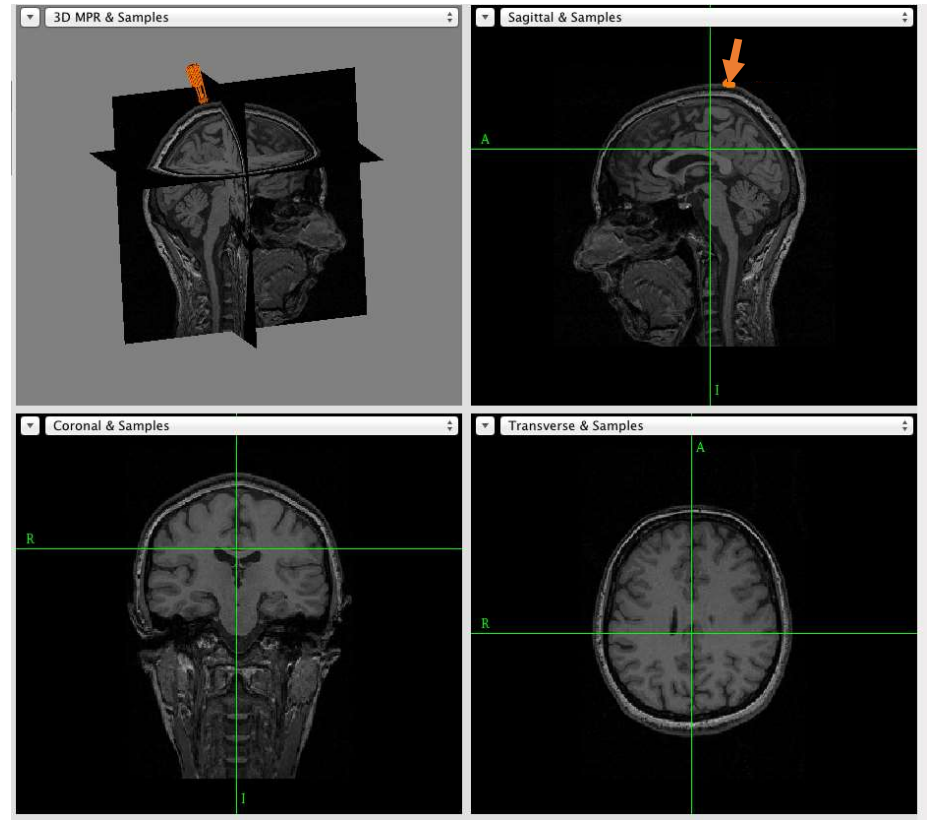

Figure 27: Screenshot showing a review of a Brainsight TMS session for a single participant. Stimulation locations are shown in orange in the $3 \mathrm{D}$ diagram, and indicated by the orange arrow in the sagittal view, demonstrating the spread of the stimulation locations. 
Within the data, the MEPs identified exhibited a variety of shapes, and the shape of the MEP curves for each muscle being stimulated was consistent for stimulations within the same phase of the step cycle. This is likely due to the specific population of neurons being activated, but may be indicative of specific pathways [37]. Likewise, the same stimulation evoked different responses in different muscles, as demonstrated by the MEPs due to the same stimulation shown in Figure 28.
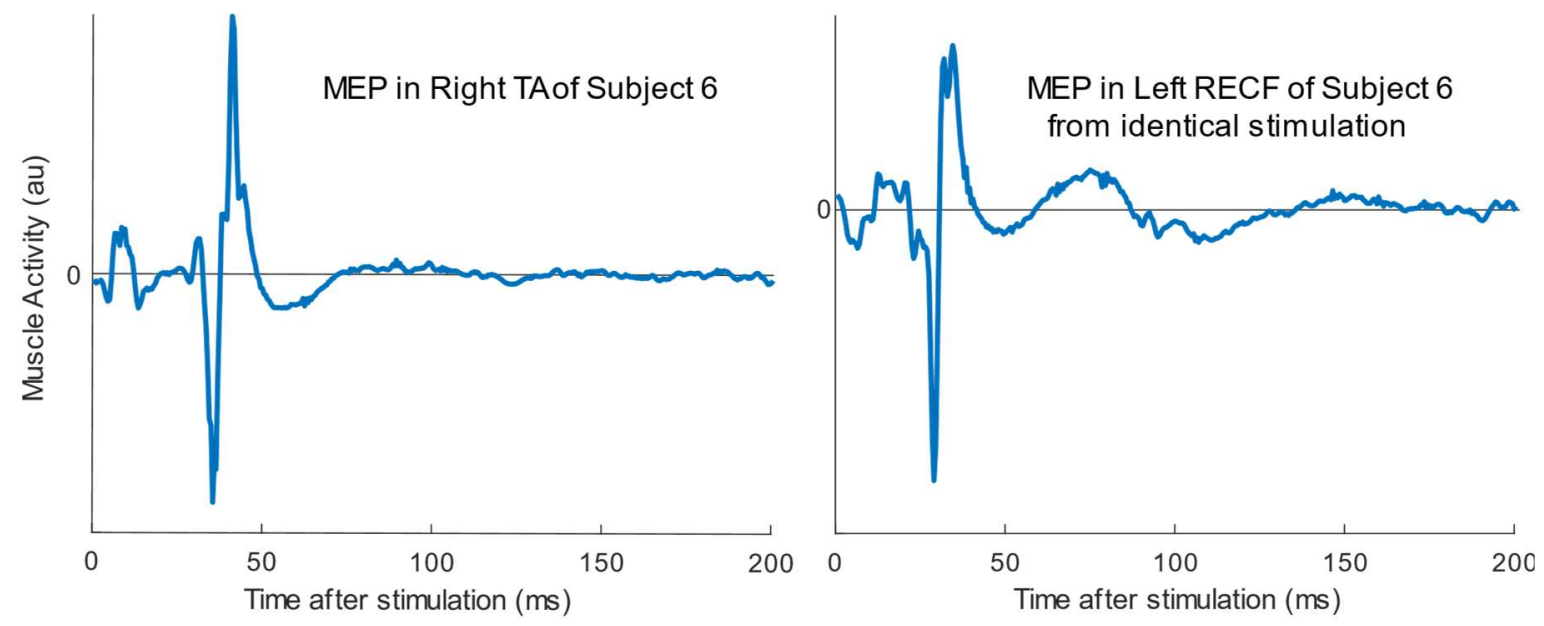

Figure 28: Raw EMG data for the same MEP epoch in two different muscles.

MEPs were also larger when the muscles being stimulated were more active, as shown below in Figure 29. For relatively larger MEPs, a cortical silent period (CSP) usually followed. This period has been interpreted as evidence of activation of inhibitory pathways [31].

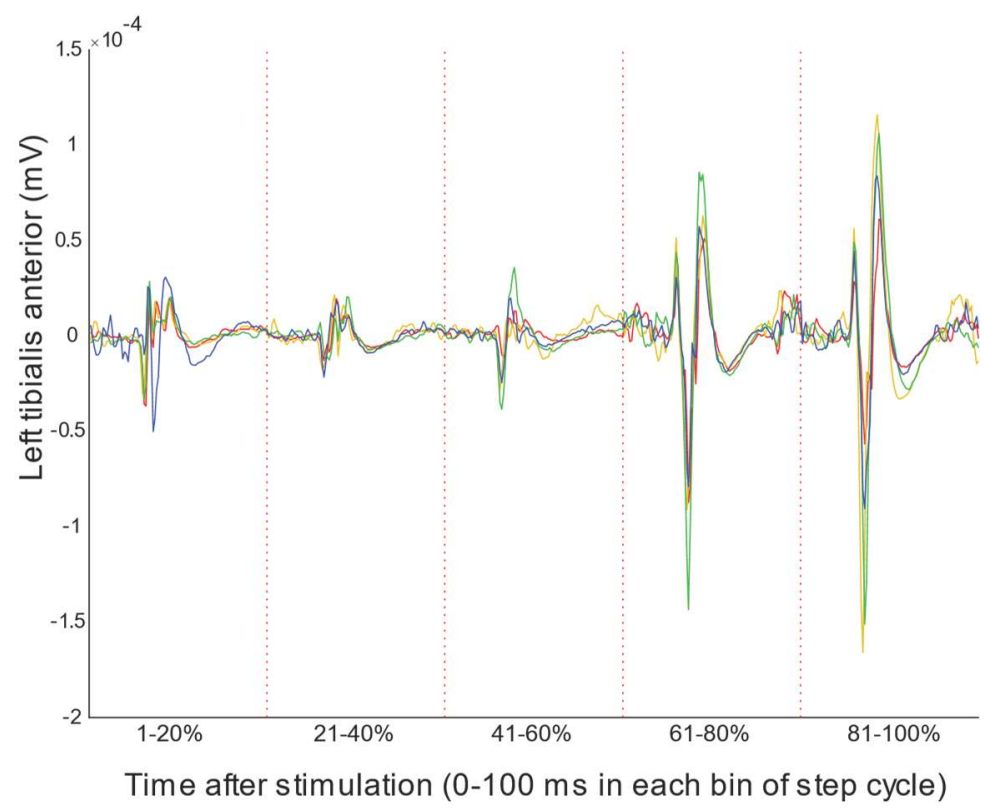

Figure 29: An example of average MEP traces separated into each bin, or 20\% of the step cycle.

The latency (or onset) of the MEPs propagated upwards from proximal to distal, as dictated by the conduction velocity unique to each participant. That is, the muscle activity evoked by the stimulation started earlier for muscles innervated closer to the brain. Figure 30 below shows the average EMG traces 
for MEPs in the five muscle signals recorded for the left leg. The signals are ordered from most proximal, to most distal. Note that the onset of the response increases the further the muscle is innervated from the brain, at a delay proportional to the conduction velocity of signals from the brain.

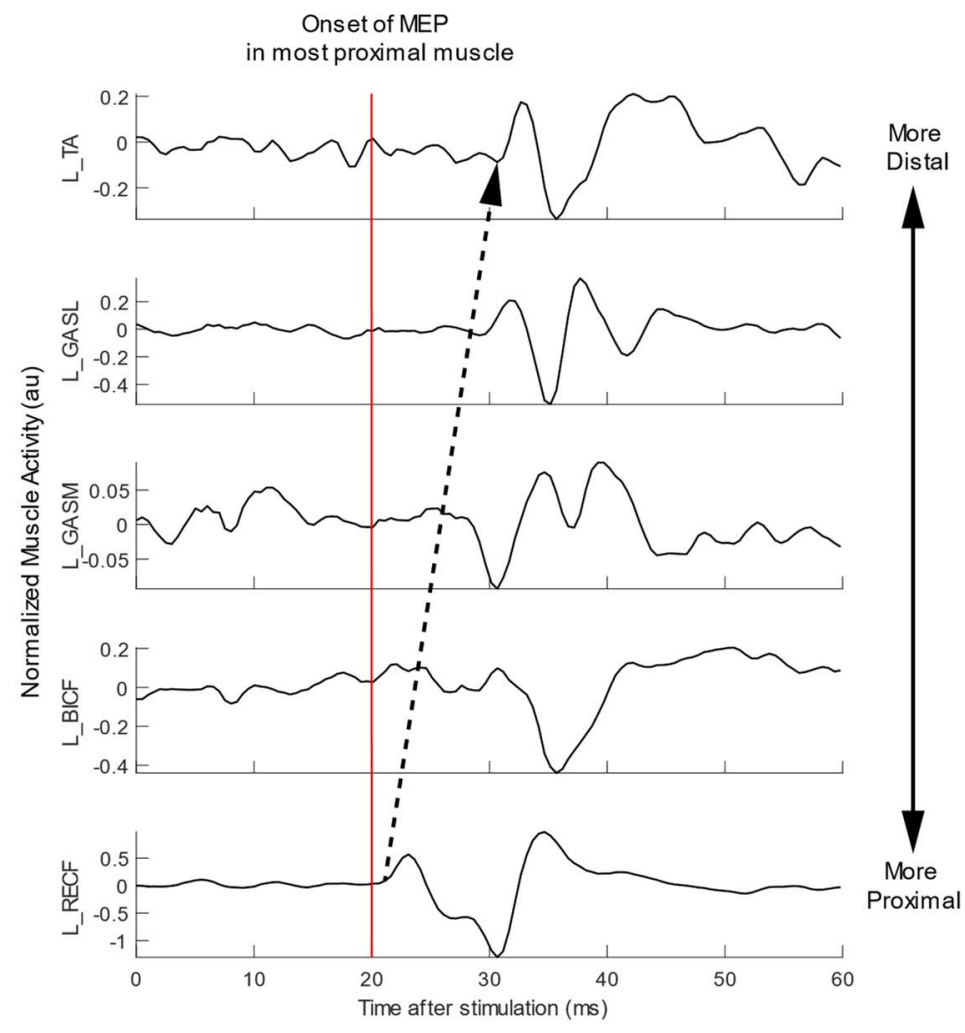

Figure 30: Average EMG traces for all MEPs for Subject 1, Trial Type 2, bin 4 of the step cycle.

The selected measure MEP magnitude, peak-to-peak amplitude, was compared to the muscle activity prior to stimulation for each MEP.

The remaining results presented are aggregated measures of MEP peak-to-peak magnitude.

\subsubsection{Cross-Subject Trends}

Normalized MEP magnitudes were summarized across participants to conduct statistical tests of significance for difference between means of responses from different conditions, and phases of the step cycle. Table 5 shows the ANOVA results for the normalized $\mathrm{MEP}_{\mathrm{pp}}$ magnitude for all included MEPs, for all participants. Note that there is a significant difference between means of MEPs evoked from stimulations in different phases, or bins, of the step cycle for all five common muscles. For this reason, subsequent analyses were separated by phase of the step cycle prior to running statistical tests.

Table 5: ANOVA summary for normalized $\mathrm{MEP}_{\mathrm{pP}}$ magnitude.

\begin{tabular}{|c|c|c|c|c|c|c|c|c|c|c|}
\hline \multirow[t]{2}{*}{ p-values } & \multicolumn{2}{|c|}{$\begin{array}{c}\text { Tibialis } \\
\text { Anterior }\end{array}$} & \multicolumn{2}{|c|}{$\begin{array}{c}\text { Lateral } \\
\text { Gastrocnemius }\end{array}$} & \multicolumn{2}{|c|}{$\begin{array}{c}\text { Medial } \\
\text { Gastrocnemius }\end{array}$} & \multicolumn{2}{|c|}{$\begin{array}{l}\text { Biceps } \\
\text { Femoris }\end{array}$} & \multicolumn{2}{|c|}{$\begin{array}{l}\text { Rectus } \\
\text { Femoris }\end{array}$} \\
\hline & Left & Right & Left & Right & Left & Right & Left & Right & Left & Right \\
\hline Left Limb Velocity & 0.00 & 0.92 & 0.97 & 0.00 & 0.00 & 0.54 & $\overline{0.02}$ & 0.18 & 0.35 & 0.00 \\
\hline Right Limb Velocity & 0.58 & 0.00 & 0.72 & 0.00 & 0.01 & 0.32 & 0.00 & 0.25 & 0.11 & 0.00 \\
\hline Binof St ep Cycle & 0.00 & 0.00 & 0.00 & 0.00 & 0.00 & 0.00 & 0.00 & 0.00 & 0.00 & 0.00 \\
\hline
\end{tabular}


Muscles were also split into muscle groups according to the bursting activity identified in 3.3.2.1 to test whether CSE to the muscle groups is modulated differentially. The cross-subject MEP magnitude trends are presented for the five common muscle signals collected in both legs first.

\subsubsection{Cross-subject Trends by Muscle}

For these analyses, the five common muscles monitored for each limb were compared. The MEPs normalized to the maximum muscle activity recorded for each participant for that muscle are presented in Figure 31.
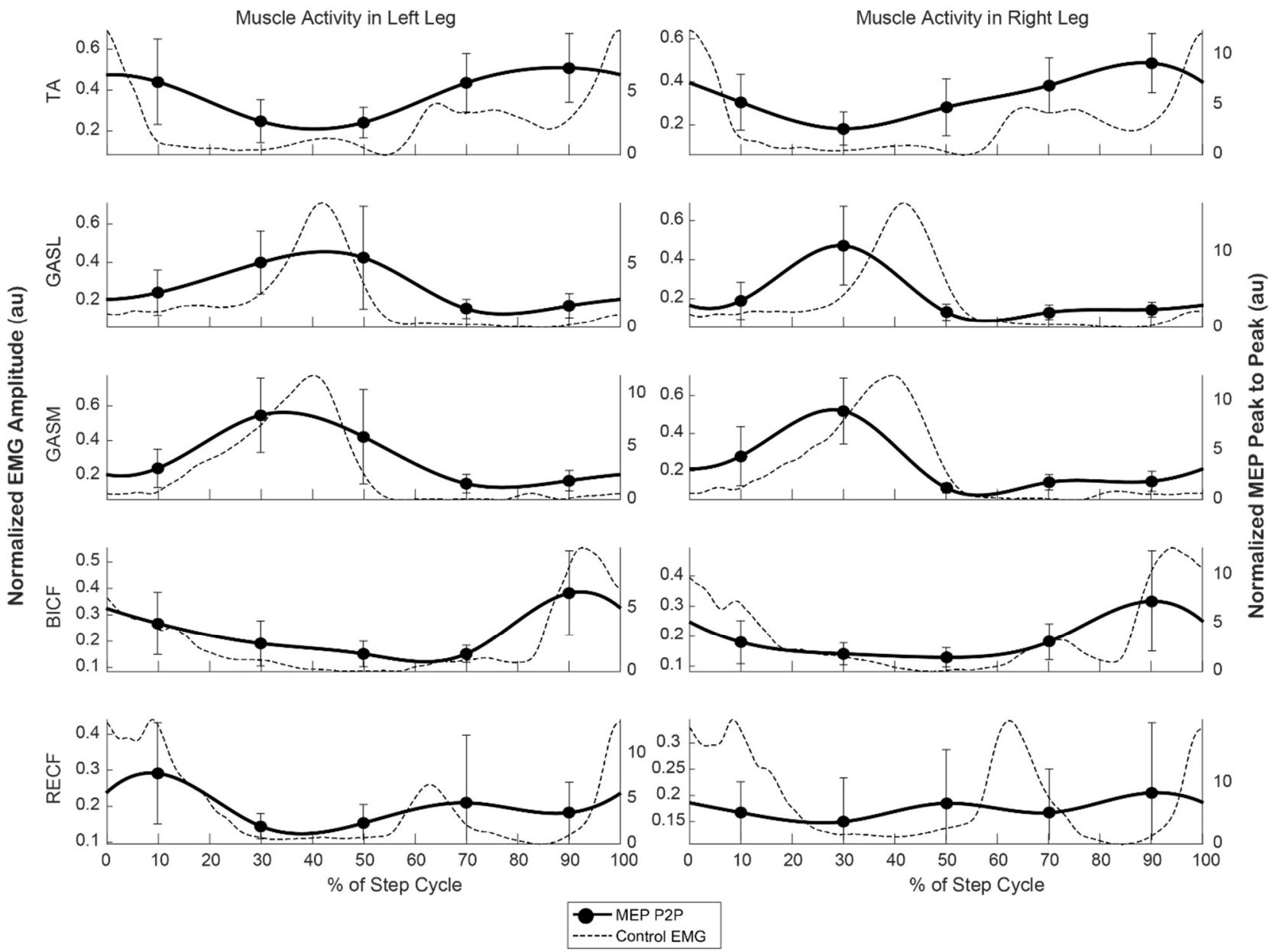

Figure 31: MEP peak to peak amplitudes (black circles), normalized to maximum EMG amplitude within each signal.

Mean EMG activity across all participants is plotted in black.

The average MEP magnitude in each muscle was correlated with the mean rectified muscle activity for each muscle, and exhibits similar trends in both legs. The standard deviation of MEP magnitude positively correlates with the peak-to-peak magnitude. 

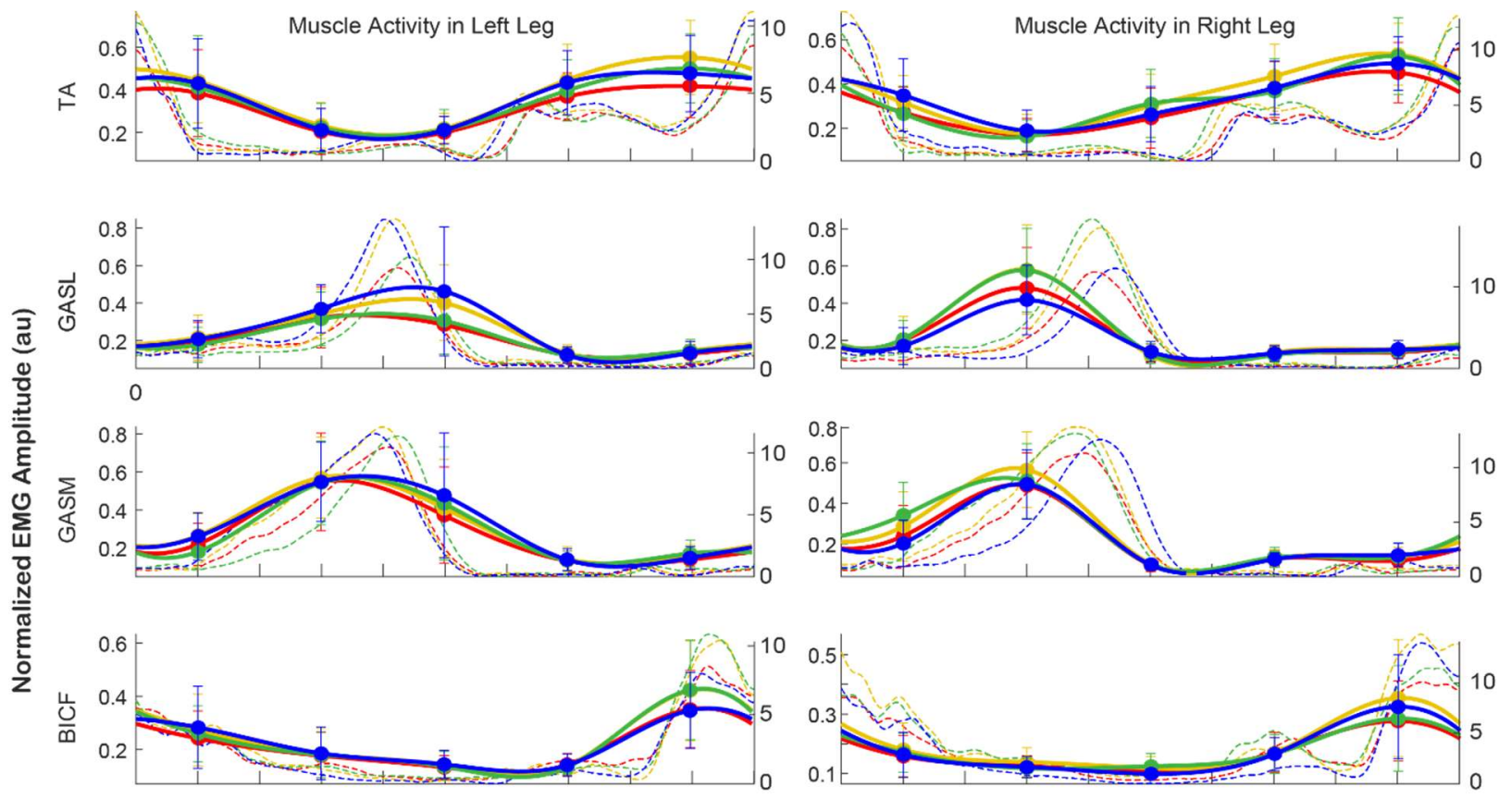

10

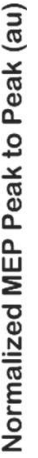
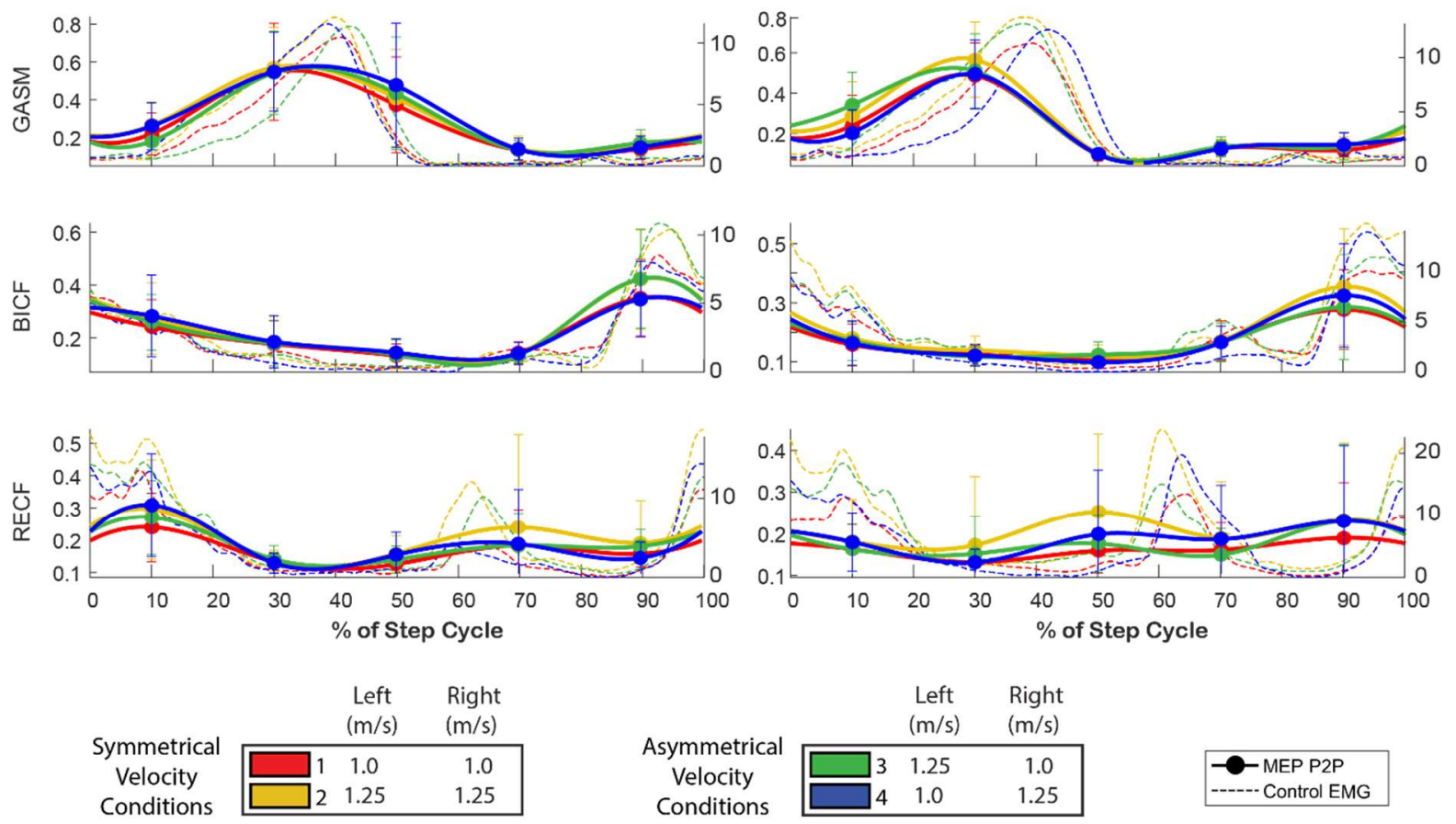

Figure 32: MEP peak to peak amplitudes (black circles), normalized to maximum EMG amplitude within each signal.

Mean EMG activity across all participants is plotted in black.

These same MEP curves are plotted according to velocity condition in Figure 32 above. Although there are differences at some phases of the step cycle, the relationship is not clear due to the spread of MEP magnitudes. 
The MEPs normalized to the muscle activity directly prior to stimulation are presented Figure 33. The trend here is not as clear, although the MEP magnitude appears to lead muscle activity. This may be due purely to dividing by larger muscle activity. Additionally, the standard deviation of this MEP magnitude is also larger than it is for the maximum EMG signal normalized MEP magnitudes.
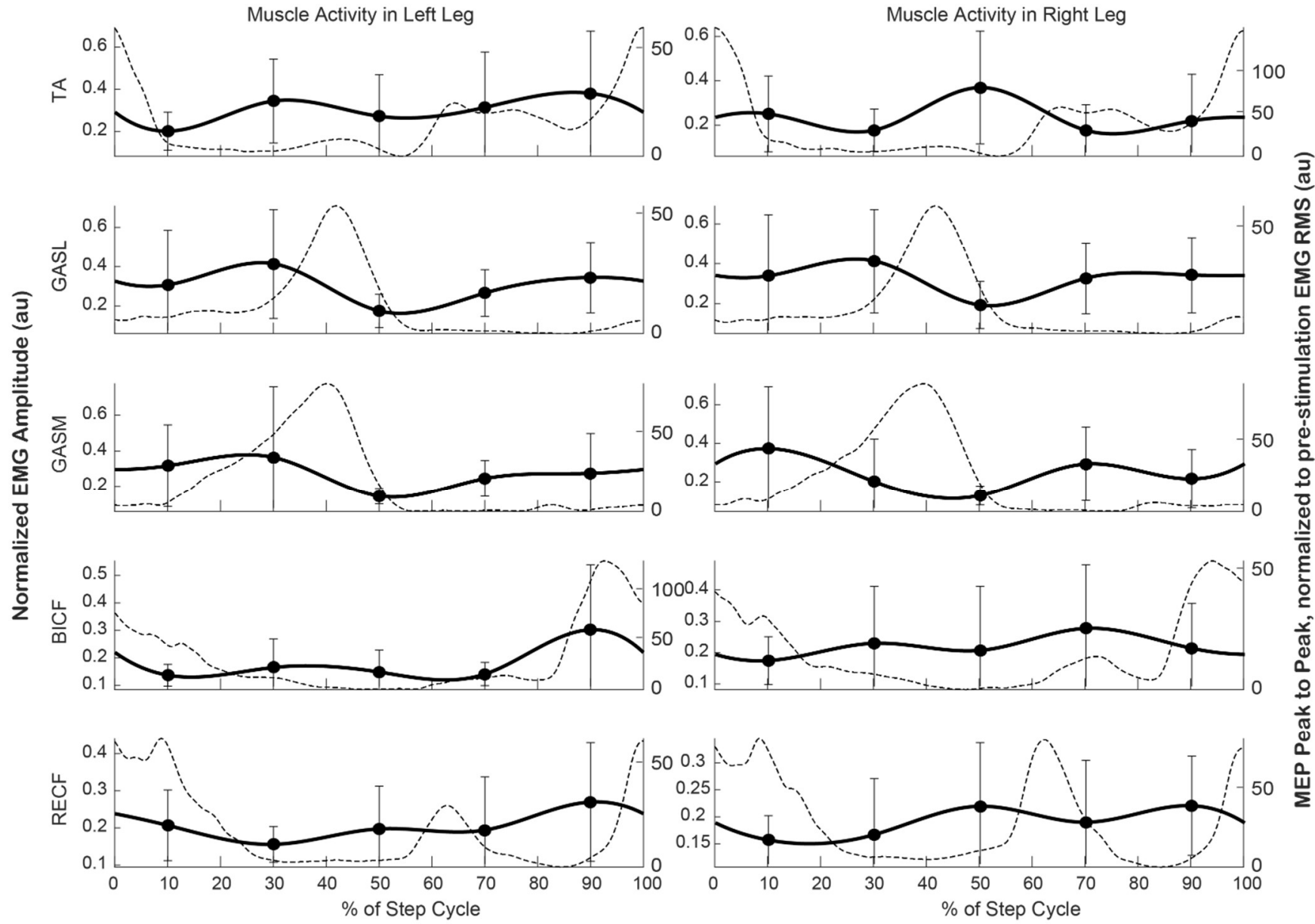

MEP P2P

Control EMG

Figure 33: MEP peak to peak amplitudes for all participants, normalized to muscle activity prior to stimulation.

Mean EMG activity across all participants is plotted in black. 

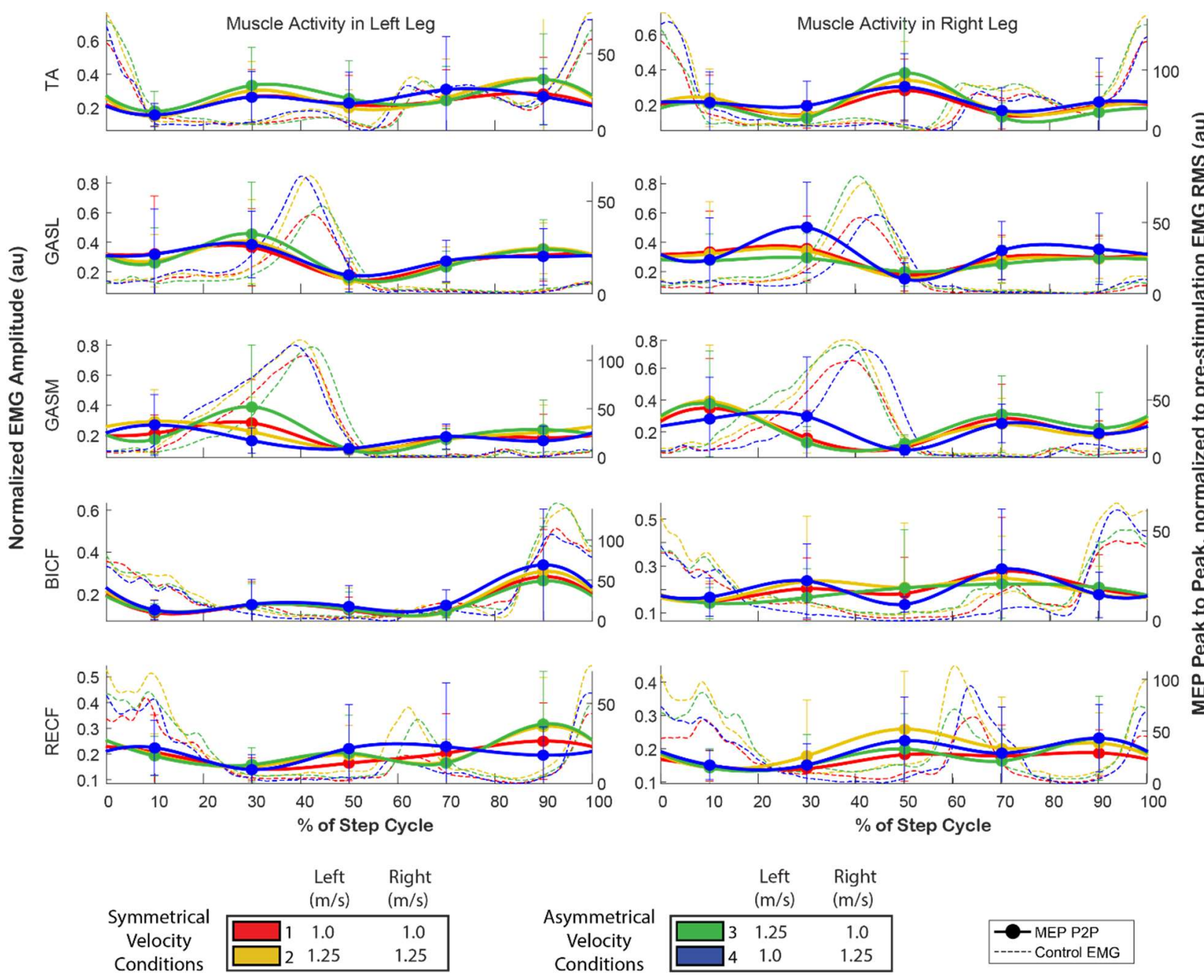

MEP P2P

Control EMG

Figure 34: MEP peak to peak amplitudes for all participants, normalized to muscle activity prior to stimulation.

Series are separated by velocity condition.

The MEP peak to peak magnitude separated by velocity condition is presented for the five common muscle signals recorded in both legs, for all participants, in Figure 34. Again, differences between velocity conditions are not clear from the plots.

\subsubsection{Cross-subject Trends by Limb Velocity in Muscle Groups}

The normalized MEP magnitudes were also plotted across the step cycle according to muscle groups, and limb velocity. The muscle groups were determined using the adaptive threshold crossing method for muscle burst detection [35] and were used to determine if the effect on corticospinal excitability was different between flexors and extensors.

The resulting series for left limb velocity are presented in Figure 35. The resulting series for right limb velocity are presented in Figure 36. 

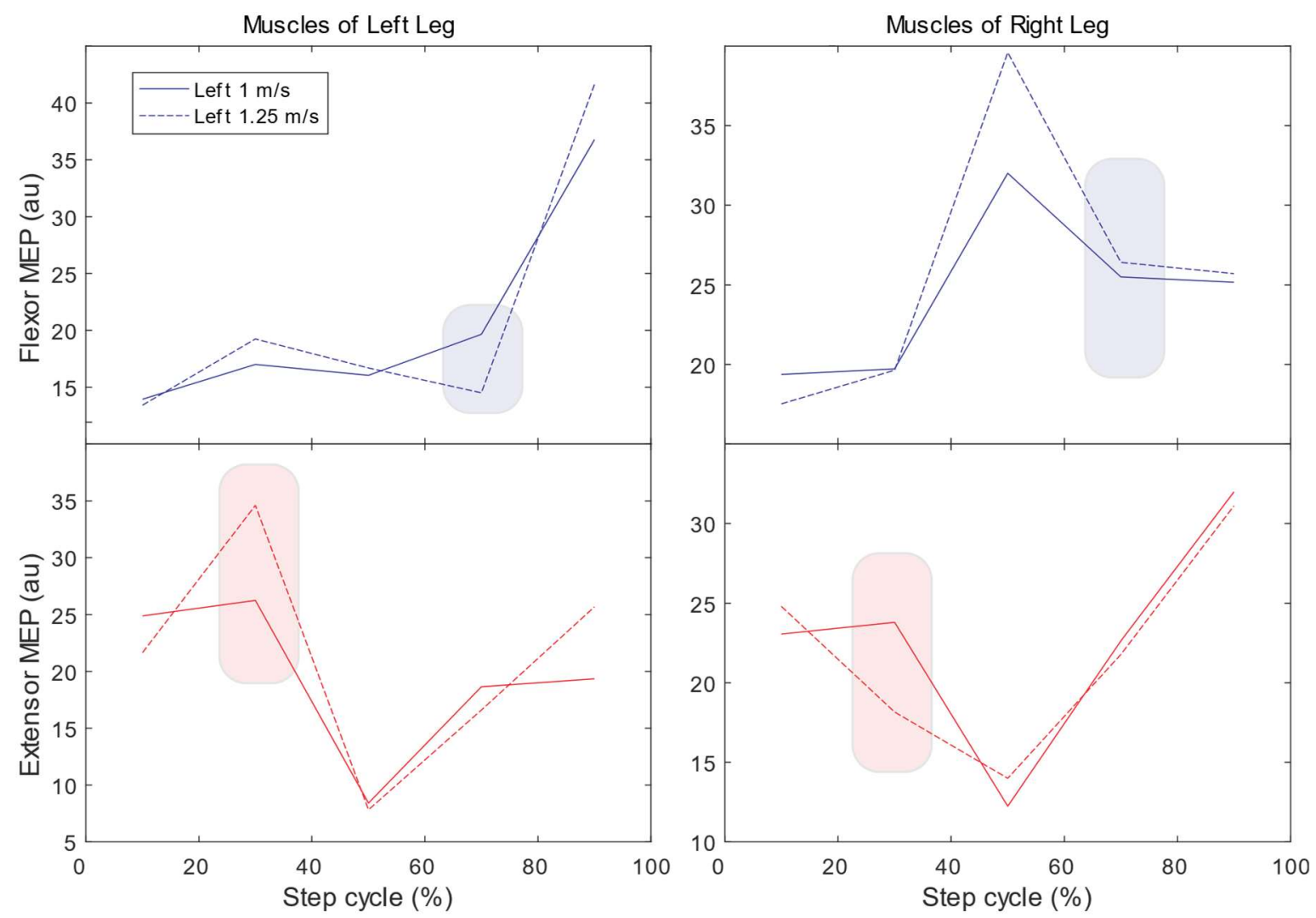

Figure 35: Normalized MEP magnitudes in muscle groups of each leg, separated by left limb velocity.
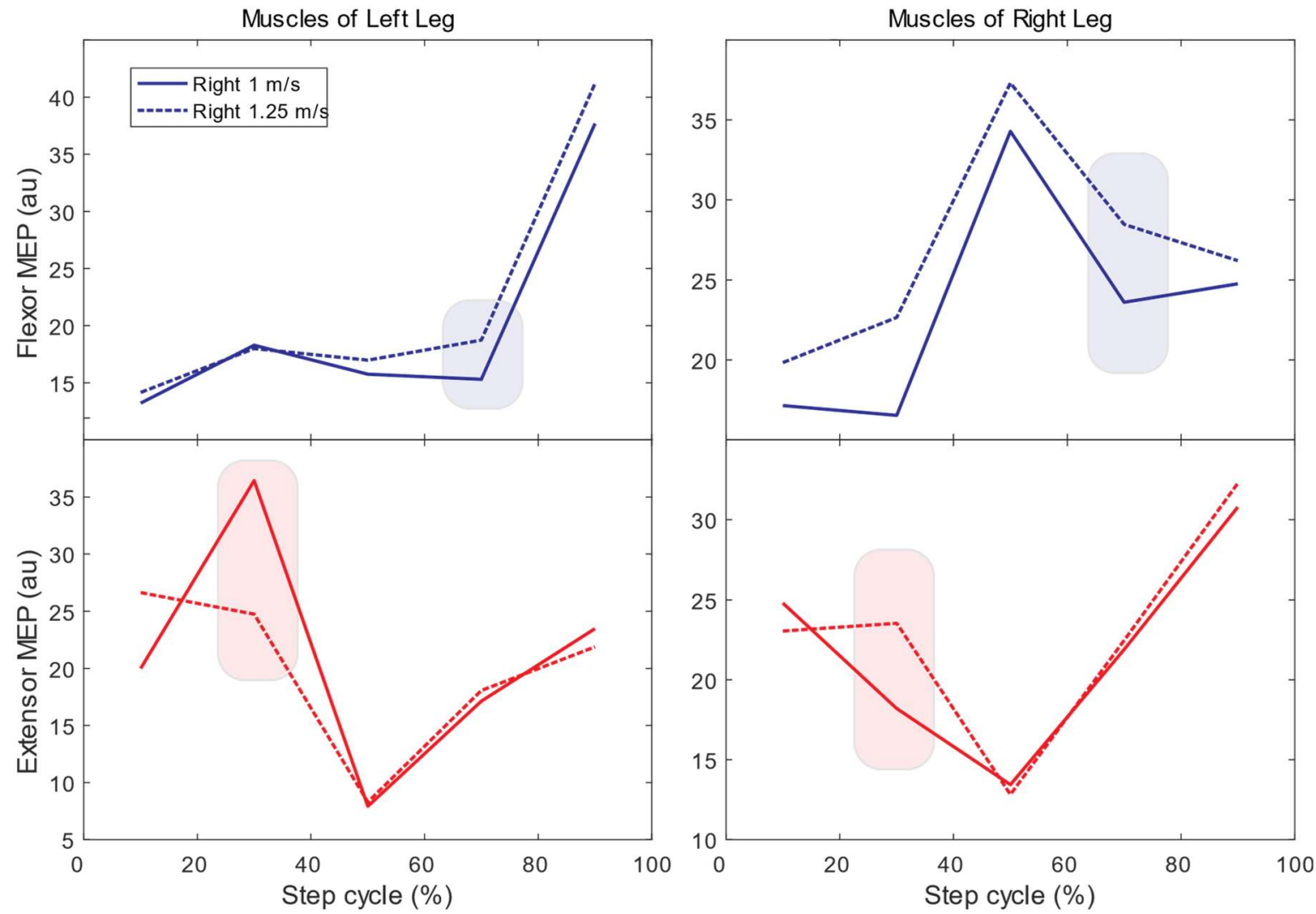

Figure 36: Normalized MEP magnitudes in muscle groups of each leg, separated by right limb velocity. 
For this analysis, a more prominent effect was found in extensors when they were stimulated between $20-40 \%$ of the step cycle, when they are most active. An effect also seemed plausible for flexors between $60-80 \%$ of the step cycle, when that group is most active. Additional statistical tests were conducted to determine if the effects of individual limb velocity were significant for these phases of the step cycle, for both muscle groups.

The main effects were significant for stimulations between $20-40 \%$ of the step cycle, and exhibit a symmetrical behavior, as seen in Figure 37. That is, increased velocity for the left belt resulted in significantly larger responses in the left leg, and significantly smaller responses in the right leg, and vice versa.
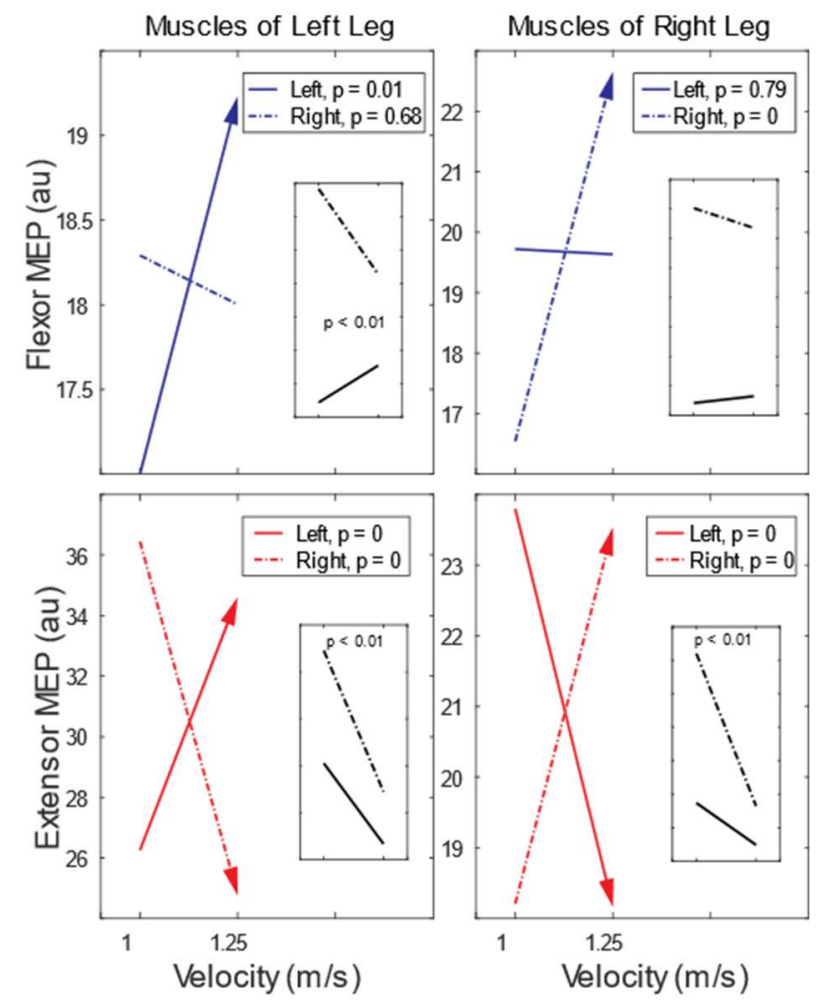

Figure 37: Plots of main effects of limb velocity on MEP magnitudes by muscle group for Bin 4: $21-40 \%$ of the step cycle. Inset plots show interaction between limb velocities.

There is also evidence of interaction between the right and left limb velocities. For example, the magnitude of the effect of the left belt velocity on left leg muscle responses depended on the right belt velocity.

Belt velocity also had a significant effect on the flexors of the same limb during this stage of the step cycle. In both cases, the response evoked in the muscles of each limb increased with increasing belt velocity of that limb. There was no effect for the contralateral limb in both cases.

The main effects of limb velocity on MEP magnitude were significant for stimulations between 61 $80 \%$ of the step cycle, as shown in Figure 38. When the right belt velocity is higher, the average MEP magnitude for flexors is significantly higher in both the left and right legs. When the left belt velocity is higher, there is no significant effect in the MEPs of the right leg, but the MEPs in the flexors of the left leg are significantly smaller. 

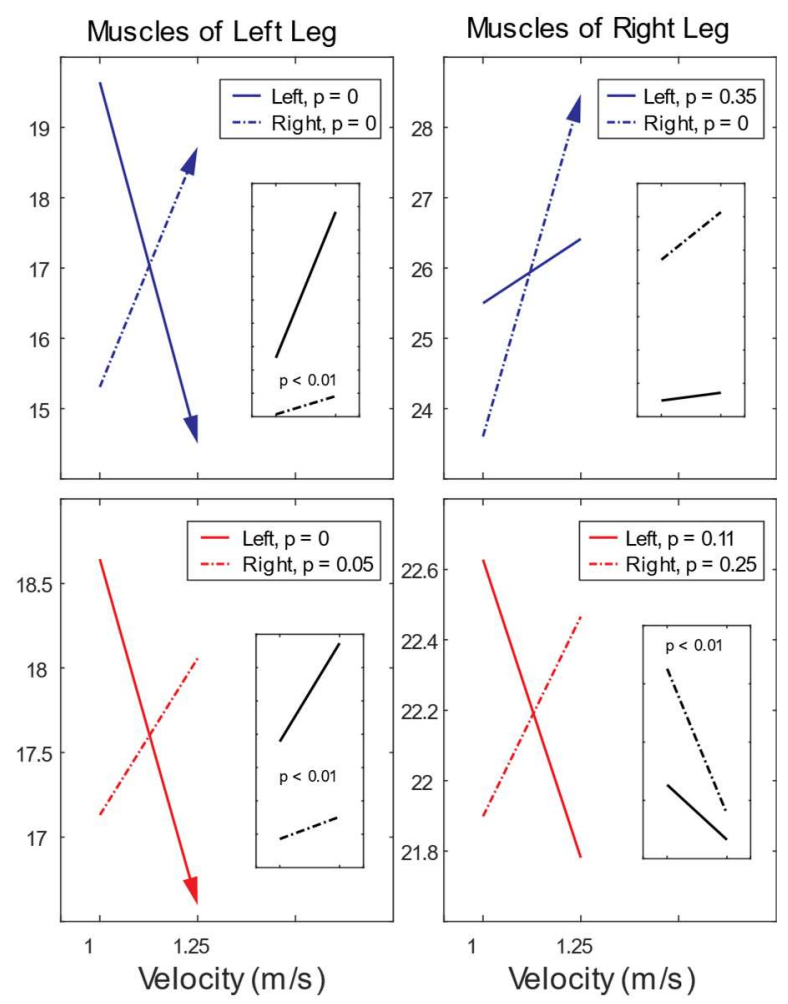

Figure 38: Plots of main effects of limb velocity on MEP magnitudes by muscle group for Bin 4: 61-80\% of the step cycle. Inset plots show interaction between limb velocities.

Combining these series demonstrates the overall symmetricity of the effect of limb velocity on extensors during stance, as shown in Figure 39.
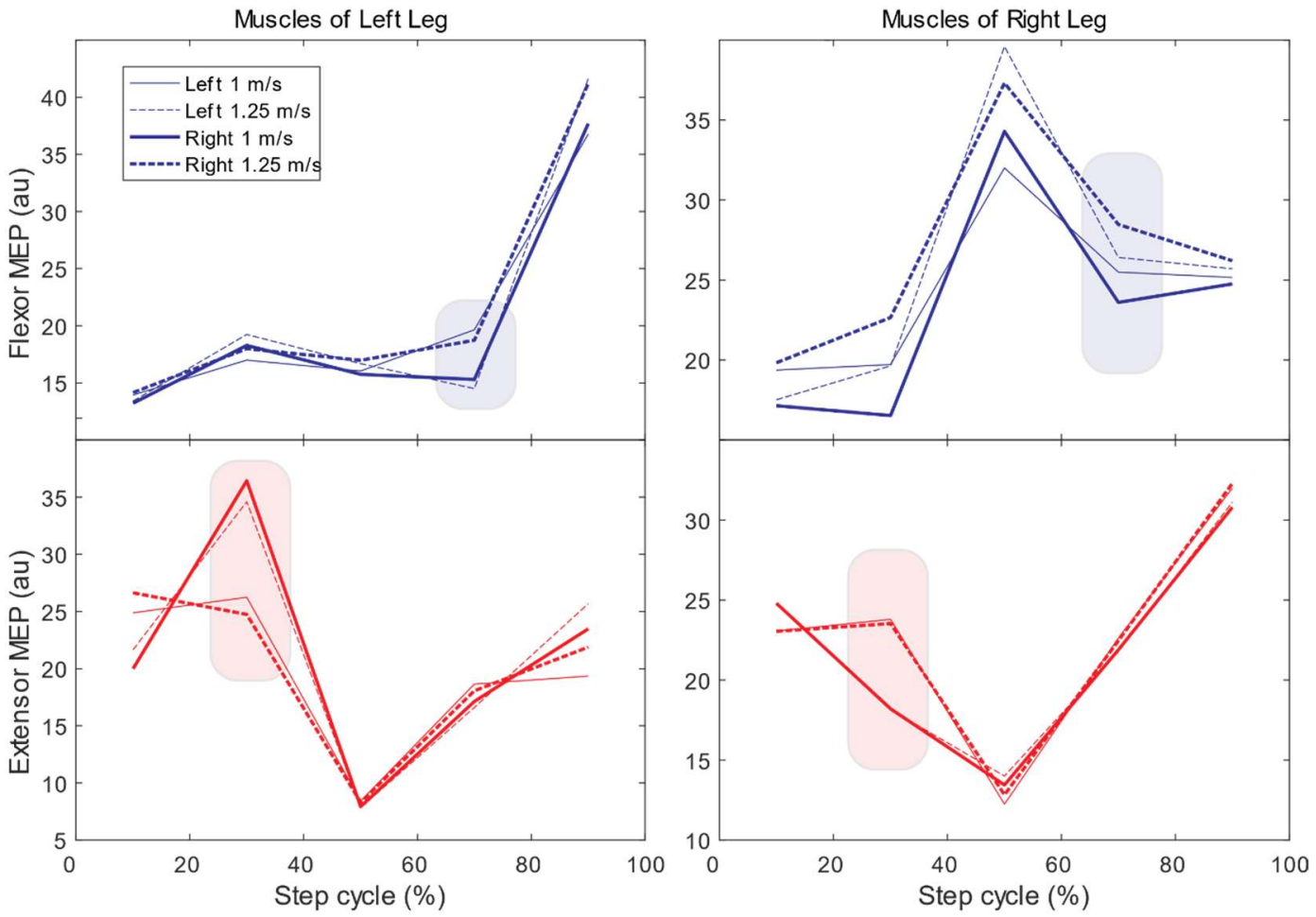

Figure 39: Normalized MEP magnitude plotted at each phase of the step cycle. 


\subsection{Discussion}

The main result of this study is there is evidence that limb velocity influences corticospinal excitability in humans during locomotion, for specific phases of the step cycle. The results for muscle activity and ground reaction forces were expected, and indicate that the data is consistent with prior locomotor studies [38]. There is evidence supporting to a decision reject the null hypothesis in certain muscles. The means of MEP magnitude between different velocity conditions have a statistically significant difference. The ANOVA conducted for pre-stimulation RMS EMG normalized MEPs that

were not excluded according to the criteria described in 3.2.8.1, yielded significant differences $(\mathrm{p}<0.001)$ between means for MEPs due to stimulations at different phases of the step cycle (see Table 5 on page 34).

This result provides converging evidence with the study of Galbreath et al [6], that limb velocity is expressed within the processing of visuomotor pathways in humans. The velocity command is then transformed into the appropriate oscillatory activity of muscle groups, as predicted by simulations using CPG models [4], [39]. To reproduce the relative proportion of stance phase to swing phase, a CPG model creating phasic muscle activity requires a larger input with increasing velocity. Additionally, although the relative proportion of time spent in swing increase in proportion to velocity, the absolute time spent in swing is weakly correlated with velocity [4]. This may explain why effects of increasing limb velocity are not prominent in flexors generating swing.

The alternative explanation is that individual muscle groups are directly controlled at the high levels of the CNS. The CSE reflecting this control would also then be velocity-dependent, reflecting the dependence on position, velocity, or force of muscles generating movement. However, this alternative nonhierarchical system is not supported by known anatomy and would suggest an even more complicated control mechanism extending into the from execution pathways to other pre-motor planning pathways within CNS. The principle of Occam's razor would dictate the proposed velocity hypothesis to be true.

\subsubsection{Velocity Signal}

The effect of the belt velocity on MEP magnitude indicates that velocity condition may influence the corticospinal excitability depending on the phase of the step cycle. This result is in accordance with the results of CPG modeling solutions for recorded data in cats, and the limb velocity discrimination task in humans introduced in the Background section. The CPG modeling result of Yakovenko et al can also reasonably explain why the effect of limb velocity is observed in during stance, but not during swing. The swing phase time of the step cycle does not exhibit as large an absolute change with increasing velocity, but the stance phase time changes substantially [4].

The differential effects observed in mean MEP magnitude of extensors and flexors throughout the step cycle agree with a prior study that measured muscle activity modulation at different velocities, conducted by Buurke et al. The results of this study suggested that there is a synergistic structure to speed dependent muscle activity modulation during locomotion in humans [18]. That is, when the activity of multiple muscles is modified according to the speed of locomotion, the gain and activation of all muscles can be represented by the combined activity of a smaller number of activations. CSE may correlate to this modular control of gait, however further study would be required to test this hypothesis. 
Visual feedback can affect CSE or even be a driving signal in the M1 as evident from the modulation of MEPs by appropriate visual feedback [23]. Visual information is known to be a driving signal of motor actions [40] and the hypothesis of this study is not contradictory to idea that the velocity command is a part of the heading direction control system. The curved path resulting from a change in heading direction can be produced by a difference in leg speeds. For example, the gentle change in the direction causing turning to the left is produced by the left limb stepping with shorter strides than the right limb in the same step cycle duration. Thus, the left limb has a slower speed than the right limb. This speed differential encoding is supported by the finding of alternative modulation of left and right extensors. In simpler animals like a common fly, the optical flow and, specifically, the velocity of optical flow can directly control the heading direction [41]. This high-level control principle is also consistent with our findings and may point to the conservation of homologous control structures across different species; however, there is an evolutionary distance of 250 to 300 million years between these species [42].

Cognitive function of any kind can also affect CSE [43]. Again, this is unlikely to create a measurable effect within this experimental protocol, because the effect would have to be persistent for the length of a trial, and happen to have no affected the remained of trials, for all participants in the same way. This is therefore unlikely to have been a contributing factor.

Neuromodulatory drugs are unlikely to have affected the CSE of participants, as the use of drugs was part of the medical exclusion criteria to remove compounding factors.

The location of stimulation between trials may have influenced the CSE in such a way as to account for the change in CSE as measured by the difference in MEP amplitude across trials. The standard deviation of the target error for all stimulations across all participants indicates that the precision of stimulation location was less than $2.0 \mathrm{~mm}$.

These effects may also be specific to treadmill walking. The following section comprises a discussion of treadmill versus above ground walking, and whether the results are specific to treadmill walking.

\subsubsection{Effect of treadmill vs. above ground locomotion}

Treadmills are used in studying biomechanics due to their size and ability to maintain subject position while completing locomotor tasks such as walking or running. Treadmills also allow for velocity to be controlled accurately, and artificially asymmetrical conditions can be applied with the use of split-belt treadmill, as was done for this study. For the current state of TMS technology, it is especially important that the participant remain in roughly the same position, and so it is not possible yet to conduct these sorts of studies using above ground walking. However, this may not be necessary. The following discussion explains why it is unlikely that the experimental set-up affected the CSE in a way as to confound the effect of the factors of interest, the forward limb velocity of each limb.

An early article by Schenau explored the biomechanics of treadmill versus overground walking by calculating energy usage for a few theoretical examples. The results of this paper suggest that as long as the belt speed is constant, a coordinate system that moves with the belt can be used, and in that frame there are no mechanical differences as compared to overground locomotion with respect to a fixed frame. Thereby, differences between treadmill and above ground locomotion cannot solely be attributed to 
varying mechanics [44]. Schenau suggested that the visual feedback was more likely to create a difference between the two types of walking, due to the importance of visual information in the regulation of posture.

Lee et al, among other groups, measured the biomechanics of overgound and treadmill gait directly to quantify the size of differences for symmetrical walking [45]. Their results indicated that while limb kinematics and spatiotemporal gait parameters remain the same, muscle activation patters and thus joint moments and powers are modified in treadmill walking as opposed to overground walking. In discussing the factors that lead to these differences, Schenau's calculations were cited, and suggested that part of the variance in muscle activity during stance can be the slight drop in belt speed at heel contact, although this would not explain variance observed later in the step cycle. The authors again suggest that the optic flow pattern is the factor with the strongest effect, as humans will try to preserve kinematic patterns during locomotion, and the influence of visual information on stride length during human locomotion has been demonstrated [46]. This factor may certainly affect the results in this study, since no optical flow was provided for the participants during locomotion.

As to the effects of asymmetrical walking on a treadmill versus overground, there are not as many articles describing the differences. One study explored how individual gait asymmetry manifested as a function of age. Nagano et al showed that effects of treadmill walking were more prominent in older participants as opposed to young participants (mean 74.0 versus 21.9 years of age). Specifically, the proportion of time spent in double support increased when treadmill walking, and this effect was not observed in young participants. The preferred speed for overground and treadmill walking speed was within $0.03 \mathrm{~m} / \mathrm{s}$ for young participants as well, at $1.23 \mathrm{~m} / \mathrm{s}$ and $1.26 \mathrm{~m} / \mathrm{s}$, respectively [47]. As a secondary conclusion in one of the studies discussed above, Lee et al found there were no differences for participants at mean age 56.0 years of age [45]. Because all participants in this study were under the age of 30 , this result suggests that the treadmill walking of participants was not significantly different from their overground walking, although this was not directly measured in this study.

\subsubsection{Improvements and Future Work}

There were methodological issues that made this experiment difficult to conduct. Despite improvements made to the stability of the coil with the apparatus described, this study relied on the skill and attention of the operator. A fully supported coil harness system could provide even greater consistency. The compromise would be for the comfort of the participant, as a greater amount of force would need to be applied to the scalp to maintain the coil position without operator oversight and continual adjustment throughout each trial.

The analytical pipeline for this study was well coordinated for the basic segmentation of data signals, however the processing of EMG data for MEP specifically was underdeveloped for the amount of data being processed. The measurement of response variables for MEPs, such as MEP $\mathrm{pp}_{\text {magnitude and }}$ integrated MEP area can be greatly improved by either sourcing technicians to help with manual feature extraction, or to perform semi-automatic feature extraction with the tools described in Chapter 4:. This semi-automatic method can include selecting appropriate features for a sample of the MEPs collected for a certain treatment (that is, each combination of muscle, velocity condition, and phase of the step cycle

for each participant) and extract the features for the remaining MEPs. Validation can be conducted using 
the mean and standard deviation of the extracted features, especially for MEP latency which has been shown to be more consistent. The trends of the MEPs were also difficult to interpret due to the sheer quantity of comparisons available.

In future studies, the collected neuronavigation data can also be used to exclude MEPs and perhaps provide a more in depths cortical mapping for analytical purposes and explanation of differences in MEP magnitude that are not explained by the treatments. 


\section{CHAPTER 4: SIGNALS PROCESSING TECHNIQUES}

In this chapter, two techniques are evaluated for use in future analyses of this and other TMS data sets.

The first technique was introduced by Zhao in his 2016 doctoral dissertation [48], and involves the use of continuous wavelet transform to identify the location of each motor evoked response within a specified time range, allowing for more accurate measurement of response variables such as the integrated area of the MEP, or the length of the cortical silent period.

The second technique focuses instead on identification of the cortical silent period using the first derivative of the filtered MEP epoch to detect onset and offset of irregular activity.

\subsection{Continuous Wavelet Transform}

This method was published in a dissertation in partial fulfillment of a doctorate in electrical engineering [48].
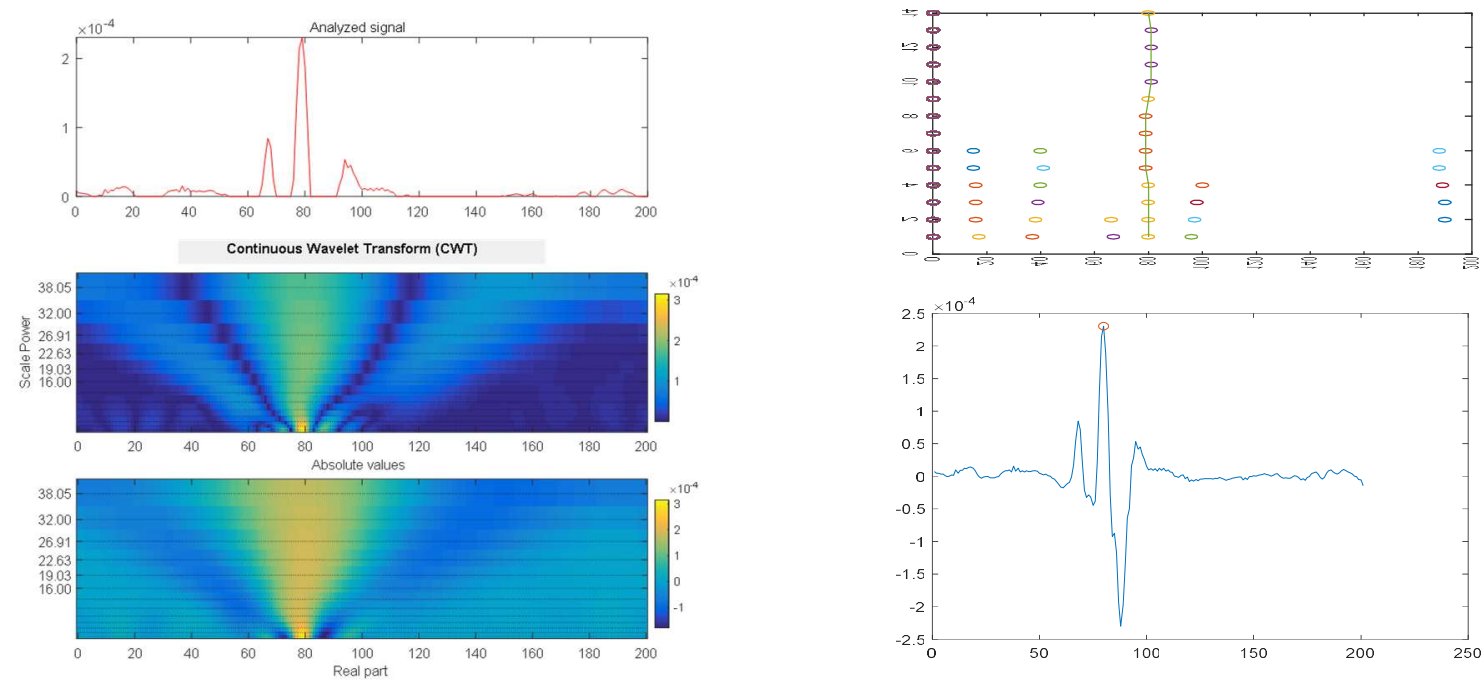

Figure 40: Example of implementation of automatic feature extraction method developed by Zhao.

The strength of this methodology is in the characterization of MEPs by attempting to determine the shape of the MEP, which can give some information as to whether and MEP is monosynaptic, or polysynaptic, for example [48].

However, some steps of this algorithm recreate existing functionality with additional steps. The builtin MATLAB peak finder can be used to achieve the same accuracy of peak finding, without running a continuous wavelet transform. Instead, the threshold for peak detection can be set based on the RMS value of the EMG data prior to stimulation.

The remaining parts of the algorithm can then be used to determine the onset of the wavelet as described. 


\subsection{Thresholding}

The cortical silent period, herein defined as the time between stimulation to return of regular muscle activity, was measured using the recorded stimulation time, and the method published by Julkunen et al in 2013 [49]:

1. The demeaned EMG data was filtered to $10-500 \mathrm{~Hz}$, and the first derivative was calculated.

2. At each time step, a logical index was calculated based on whether the value of the first derivative was greater or less than a defined threshold.

3. At each time step, the center-windowed mode of the logical indexes including and around the time step was calculated.

4. The longest period of no activity was defined as the cortical silent period for each MEP epoch.

Choose threshold based on average pre-stimulation RMS value for each signal for each participant.

This technique was used to add information to an MEP viewer created to better understand the data. An example of MEP data loaded and processed in the viewer is presented in Figure 41 below. Instead of using a fixed threshold, the RMS of the muscle activity directly prior to stimulation was used to determine the presence of a cortical silent period.

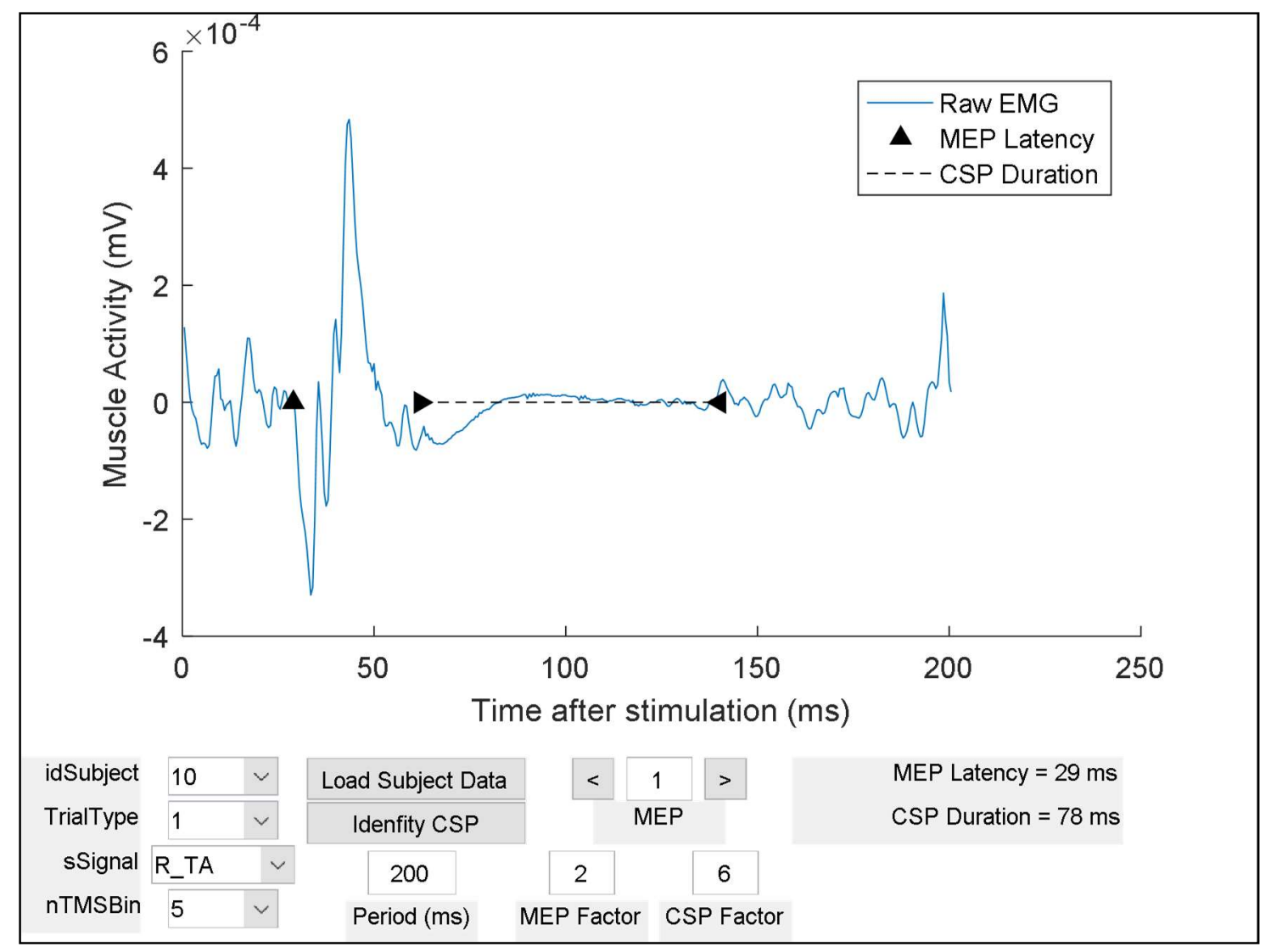

Figure 41: Example of MEP viewer, displaying detected latency and cortical silent period. 
The utility of this technique comes in its simplicity and ease of implementation. Additionally, this technique can be modified by technicians easily to aid in semi-automated feature extraction. A resulting data set can then be used to train a machine learning algorithm to vary parameters to achieve the highest accuracy for the supervised set. As with the first method evaluated, this technique works best when the signal to noise ratio is high, however it is much better for supervised feature extraction as the parameters are easier to understand.

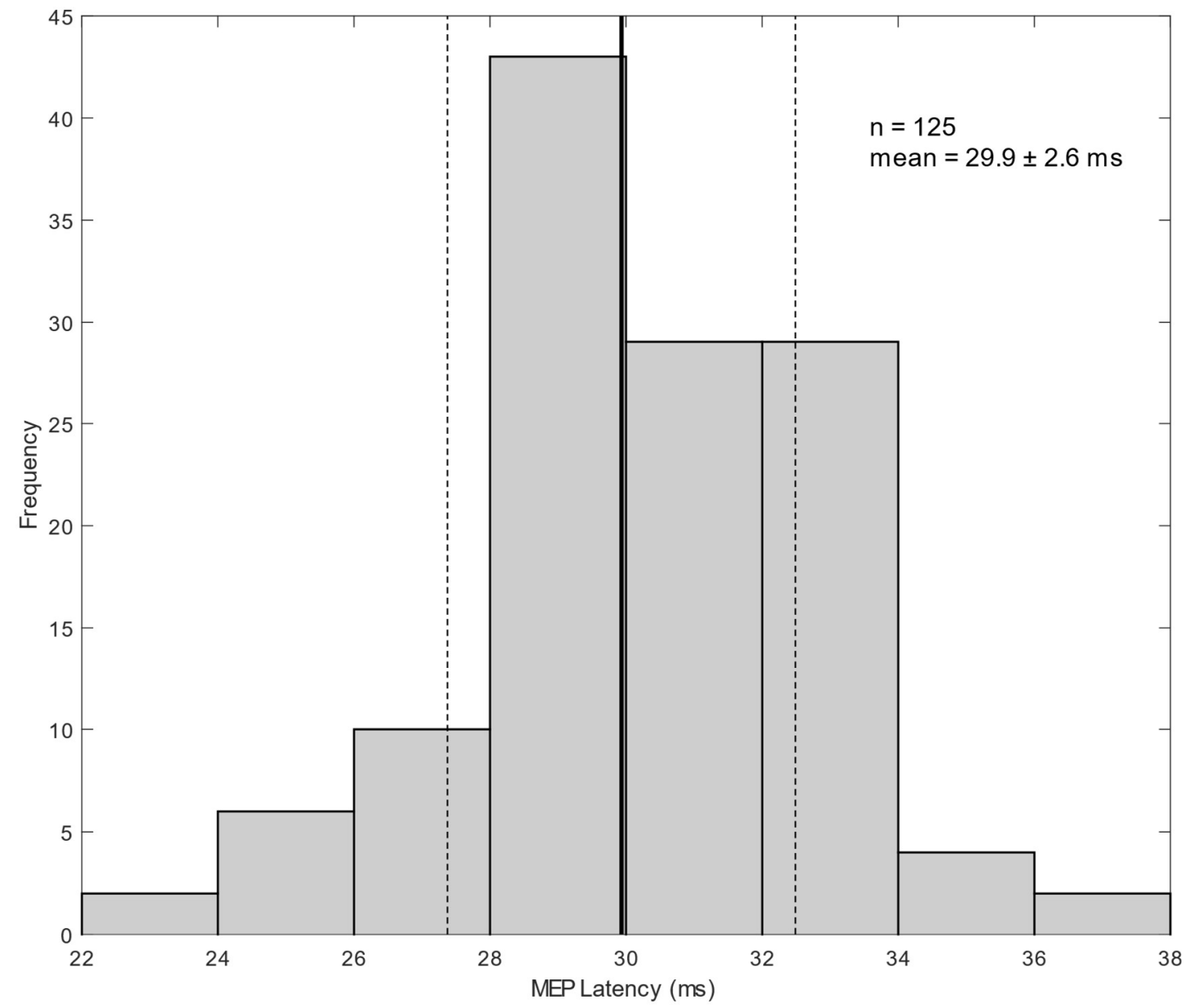

Figure 42: Histogram of MEP Latency values collected using the MEP viewer, for MEPs in the right tibialis anterior for subject 1, for velocity condition 1.

Figure 42 shows the distribution of MEP latency (the time after stimulation at which an MEP can be detected) values collected using the semi-automated method described above, and implemented in a MATLAB MEP viewer. Information about the cortical silent period can also be collected, and used to test whether the cortical silent period changes according to the velocity condition. Additionally, the MEP latency and CSP start time can be used to define the period for MEP quantification in order to compare MEP magnitude more accurately. PCA can also then be used to attempt to draw further information from the dominant waveforms found throughout MEPs of different conditions, phase of step cycle, subject, and so on, as suggested by [48] in his 2016 doctoral dissertation. 


\subsection{Summary and Suggested Use}

The two techniques evaluated had varying success. The continuous wavelet transform did not appear to offer additional information over built-in peak finding algorithms contained within MATLAB, however the algorithm evaluated did.

In future studies, it is recommended that the thresholding method be used to distill additional information from the dataset. Further tuning needs to be completed with each data set or set of experimental instruments in order to conduct completely automated feature detection of MEPs.

Using principle component analysis to identify similar features across MEPs in the same muscle is also an interesting method that may yield additional information to neuroscientists. Additional testing to determine what these features represent in the control of muscles during different tasks may help researchers interpret the shape of the MEPs more meaningfully as well. 


\section{CHAPTER 5: CONCLUSION}

\subsection{Thesis Summary}

This thesis contains a complete description of the main experiment: a TMS study conducted to evaluate CSE in humans in four walking conditions. Included is a primer on the basic concepts in the relevant biomechanics techniques and neuroscience topics required to understand the results and discussion of the experiment.

The hypothesis was that limb velocity is encoded in the internal model used by the brain to control locomotor tasks. The results indicate that individual limb velocity influences CSE during stance. This suggests that a limb velocity may indeed be incorporated in the control of locomotion.

This work, as well as the other TMS studies described in the literature review, demonstrates the difficulties in interpreting the trends in MEPs from TMS. Each experiment must be carefully planned to maximize the signal to noise ratio of the MEP to noise in the EMG signal, without saturating the MEP by using too high a stimulation intensity.

Locomotor tasks present the additional challenge of movement of the head with respect to the experimenter and TMS coil, and while this was addressed with a harness, the system still depends on the skill and attention of the operator to ensure the coil stays over the same target area over an hour of trials.

\subsection{Future Work}

Evaluation of corticospinal excitability with a finer tool will be invaluable to researchers and developers of implants that provide cortical stimulation. While TMS experiments can provide an overall measure of the state of the corticospinal excitability, when funds and/or more mature technology are available, it will be more valuable to conduct stimulation-response analysis with data collected from devices that can provide a more localized non-invasive stimulation. Additional cortical mapping prior to experimentation can also help improve the interpretability of TMS study results, however this also depends on the time, funding, and staff available for conducting the experiments.

The dataset collected will be further processed using the tools described in Chapter 4: to collect MEP latency and CSP data. The MEP latency can be used to compare the ipsi- and contra-lateral limbs for each stimulation, by comparing the latency of MEPs due to the same stimulation in identical muscles of each limb. The shorter latency MEP represents MEP due to direct stimulation (contra-lateral) and the longer latency MEP represents the ipsi-lateral response. This information may have further implications for interpreting the results of new analysis. Computational models can also be used to analyze pattern generation within the dataset.

In future studies, TMS or other non-invasive stimulation techniques can be combined with congruent or incongruent visual and proprioceptive feedback via virtual reality (VR). Physiological vibration of muscle bodies can also be used to describe the response of the CSE to perturbations. 


\section{REFERENCES}

[1] S. Grillner, J. Nilsson, and A. Thorsensson, "Intra-abdominal pressure changes during natural movements in man," Act physiol. scand., vol. 103, pp. 275-283, 1978.

[2] T. G. Brown, "The intrinsic factors in the act of progression in the mammal.," Proc. R. Soc. B, Biol. Sci., vol. 84, no. 572, pp. 308-319, 1911.

[3] S. Grillner et al., "Intrinsic function of a neuronal network - A vertebrate central pattern generator," Brain Res. Rev., vol. 26, no. 2-3, pp. 184-197, 1998.

[4] S. Yakovenko, A bierarchical perspective on rhythm generation for locomotor control, 1st ed., vol. 188. Elsevier BV., 2011.

[5] A. McCrea, David and A. Rybak, Ilya, "Modeling the mammalian locomotor CPG: insights from mistakes and perturbations,” Prog Brain Res., vol. 165, pp. 235-253, 2008.

[6] K. Galbreath, "Do humans drive spinal cord with limb velocity signal?," West Virginia University, 2015.

[7] T. Sela and M. Lavidor, "Textbook of Neuromodulation,” WVU, pp. 257-273, 2015.

[8] Z.-D. Deng, S. H. Lisanby, and A. V Peterchev, "Coil design considerations for deep transcranial magnetic stimulation.," Clin. Neurophysiol., vol. 125, no. 6, pp. 1202-12, 2014.

[9] M. Knikou, N. Hajela, and C. K. Mummidisetty, "Corticospinal excitability during walking in humans with absent and partial body weight support," Clin. Neurophysiol., vol. 124, no. 12, pp. 2431-2438, 2013.

[10] D. Barthélemy, S. Alain, M. J. Grey, J. B. Nielsen, and L. J. Bouyer, "Rapid changes in corticospinal excitability during force field adaptation of human walking," Exp. Brain Res., vol. 217, no. 1, pp. 99_ 115, 2012.

[11] I. Faria-Fortini, M. L. Basilio, A. A. Scianni, C. D. C. M. Faria, and L. Teixeira-Salmela, "Performance and capacity-based measures of locomotion, compared to impairment-based measures, best predicted participation in individuals with hemiparesis due to stroke," Disabil. Rehabil., pp. 1-8, 2017.

[12] C. S. Sherrington, "Flexion-reflex of the limb, crossed extension-reflex, and reflex stepping and standing," J. Physiol., vol. 40, no. 1-2, pp. 28-121, 1910.

[13] P. M., "L'autonomie et la centralization dans le systeme nerveux des animaux.," Trav. du Lab. la Physiol. Inst. Solvey, vol. 7, pp. 1-208, 1905.

[14] D. A. Winter, "Human balance and posture standing and walking control during," 1995.

[15] T. McGreer, "Passive Dynamic Walking," Int. J. Rob. Res., vol. 9, no. 2, pp. 62-82, 1990.

[16] and G. S. Mark L. Latash, John P. Scholz, “Toward a New Theory of Motor Synergies," J. Chem. Inf. Model., vol. 53, no. 9, pp. 1689-1699, 2013.

[17] E. Todorov and M. I. Jordan, "Optimal feedback control as a theory of motor coordination," Nat Neurosit, vol. 5, no. 11, p. 1226-35., 2002.

[18] T. J. W. Buurke, C. J. C. Lamoth, L. H. V. Van Der Woude, and A. R. Den Otter, "Synergistic Structure in the Speed Dependent Modulation of Muscle Activity in Human Walking," PLoS One, pp. 1-19, 2016.

[19] O. M. Smith, "Posicast Control of Damped Oscillatory Systems," Proc. IRE, vol. 45, no. 9, pp. 1249_ $1255,1957$. 
[20] A. D. Kuo, "The relative roles of feedforward and feedback in the control of rhythmic movements.," Motor Control, vol. 6, no. 2, pp. 129-145, 2002.

[21] A. Lundberg, Reflex Control of Stepping. Oslo: Universitetsforlaget, 1969.

[22] N. A. Bernstein, The co-ordination and regulation of movements. Oxford: Pergamon Press, 1967.

[23] F. Behrendt, M. H. E. De Lussanet, K. Zentgraf, and V. R. Zschorlich, "Motor-evoked potentials in the lower back are modulated by visual perception of lifted weight," PLoS One, vol. 11, no. 6, pp. 113, 2016.

[24] R. Kandel, Eric, H. Schwartz, James, and M. Jessell, Thomas, Principles of Neural Science, 4/e. McGrawHill Companies, 2000.

[25] Y. H. Lee and T. H. Lee, "Muscle response while holding an unstable load," Clin. Biomech., vol. 17, no. 4, pp. 250-256, 2002.

[26] J. J. Won, “An illustration of the pyramidal tract." [Online]. Available: http://jerryjwon.com/portfolio_JW_5.html.

[27] and D. Sliwinska, Vitello, "Transcranial Magnetic Stimulation for Investigating Causal Brainbehavioral Relationships and their Time Course.," J. Vis. Exp., vol. 3791, no. July, pp. 1-9, 2014.

[28] T. Paus and J. Barrett, "Transcranial magnetic stimulation (TMS) of the human frontal cortex: implications for repetitive TMS treatment of depression," J. Psychiary Neurosci., vol. 29, no. 4, pp. 268279, 2004.

[29] M. S. A. Curt and G. C. W. Berger, "Voluntary control of human gait : conditioning of magnetically evoked motor responses in a precision stepping task," pp. 583-588, 1999.

[30] N. T. Petersen et al., "Suppression of EMG activity by transcranial magnetic stimulation in human subjects during walking.," J. Physiol., vol. 537, pp. 651-656, 2001.

[31] N. T. Petersen, H. S. Pyndt, and J. B. Nielsen, "Investigating human motor control by transcranial magnetic stimulation," pp. 1-16, 2003.

[32] K. Davranche, J. Temesi, S. Verges, and T. Hasbroucq, "ScienceDirect Transcranial magnetic stimulation probes the excitability of the primary motor cortex : A framework to account for the facilitating effects of acute whole-body exercise on motor processes," J. Sport Heal. Sci., vol. 4, no. 1, pp. 24-29, 2015.

[33] S. Bestmann and J. Duque, "Transcranial Magnetic Stimulation : Decomposing the Processes Underlying Action Preparation,” 2015.

[34] C. J. de Luca, R. S. LeFever, M. P. McCue, and A. P. Xenakis, "Behaviour of human motor units in different muscles during linearly varying contractions," J. Physiol., vol. 329, pp. 113-128, 1982.

[35] S. Yakovenko, "Control of Locomotor Cycle Durations," J. Neurophysiol., vol. 94, no. 2, pp. 10571065, 2005.

[36] M. Van De Ruit and M. J. Grey, "The TMS Map Scales with Increased Stimulation Intensity and Muscle Activation,” Brain Topogr., vol. 29, no. 1, pp. 56-66, 2016.

[37] S. Bestmann and J. W. Krakauer, "The uses and interpretations of the motor-evoked potential for understanding behaviour,” Exp. Brain Res., vol. 233, no. 3, pp. 679-689, 2015.

[38] Y. P. Ivanenko, R. Grasso, V. Macellari, and F. Lacquaniti, "Control of foot trajectory in human locomotion: role of ground contact forces in simulated reduced gravity," J. Neurophysiol., vol. 87, pp. 3070-3089, 2002. 
[39] S. M. Danner, S. D. Wilshin, N. A. Shevtsova, and I. A. Rybak, "Central control of interlimb coordination and speed-dependent gait expression in quadrupeds," J. Physiol., vol. 0, pp. 1-21, 2016.

[40] M. A. Goodale, "Visuomotor control: Where does vision end and action begin?," Curr. Biol., vol. 8, no. 14, pp. R489-R491, 1998.

[41] S. N. Fry, N. Rohrseitz, A. D. Straw, and M. H. Dickinson, "Visual control of flight speed in Drosophila melanogaster.," J. Exp. Biol., vol. 212, pp. 1120-1130, 2009.

[42] R. J. Kulathinal and D. L. Hartl, “The latest buzz in comparative genomics," Genome Biol., vol. 6, no. 1, p. 201, 2005.

[43] S. Bestmann and J. W. Krakauer, "The uses and interpretations of the motor - evoked potential for understanding behaviour,” pp. 679-689, 2015.

[44] G. J. van Ingen Schenau, "Some fundamental aspects of the biomechanics of overground versus treadmill locomotion.," Medicine and science in sports and exercise, vol. 12, no. 4. pp. 257-261, 1980.

[45] S. J. Lee and J. Hidler, "Biomechanics of overground vs. treadmill walking in healthy individuals.," J. Appl. Physiol., vol. 104, no. 3, pp. 747-755, 2008.

[46] T. Prokop, M. Schubert, and W. Berger, "Visual influence on human locomotion. Modulation to changes in optic flow," Exp. Brain Res., vol. 114, no. 1, pp. 63-70, 1997.

[47] H. Nagano, R. K. Begg, W. A. Sparrow, and S. Taylor, "A comparison of treadmill and overground walking effects on step cycle asymmetry in young and older individuals," J. Appl. Biomech., vol. 29, no. 2, pp. 188-193, 2013.

[48] Z. Liu, "Electromyographic Signal Processing With Application To Spinal Cord Injury,” vol. 2016, 2016.

[49] P. Julkunen, E. Kallioniemi, M. Könönen, and L. Säisänen, "Feasibility of automated analysis and inter-examiner variability of cortical silent period induced by transcranial magnetic stimulation," $J$. Neurosci. Methods, vol. 217, no. 1-2, pp. 75-81, 2013.

[50] R. Gage, James, A. Deluca, Peter, and S. Renshaw, Thomas, "Gait Analysis : Principles and Applications Lr@ ry,” Am. Acad. Orthop. Surg., vol. 77-A, no. 10, pp. 1607-1623, 1995. 